\title{
Understanding ART Adherence
}

\author{
Citation for published version (APA):
}

Ramlagan, S. (2019). Understanding ART Adherence: Exploring the structural and psychosocial determinants of antiretroviral therapy adherence among pregnant women in Mpumalanga Province, South Africa. [Doctoral Thesis, Maastricht University]. ProefschriftMaken Maastricht. https://doi.org/10.26481/dis.20191203sr

\section{Document status and date:}

Published: 01/01/2019

DOI:

10.26481/dis.20191203sr

Document Version:

Publisher's PDF, also known as Version of record

\section{Please check the document version of this publication:}

- A submitted manuscript is the version of the article upon submission and before peer-review. There can be important differences between the submitted version and the official published version of record.

People interested in the research are advised to contact the author for the final version of the publication, or visit the DOI to the publisher's website.

- The final author version and the galley proof are versions of the publication after peer review.

- The final published version features the final layout of the paper including the volume, issue and page numbers.

Link to publication

\footnotetext{
General rights rights.

- You may freely distribute the URL identifying the publication in the public portal. please follow below link for the End User Agreement:

www.umlib.nl/taverne-license

Take down policy

If you believe that this document breaches copyright please contact us at:

repository@maastrichtuniversity.nl

providing details and we will investigate your claim.
}

Copyright and moral rights for the publications made accessible in the public portal are retained by the authors and/or other copyright owners and it is a condition of accessing publications that users recognise and abide by the legal requirements associated with these

- Users may download and print one copy of any publication from the public portal for the purpose of private study or research.

- You may not further distribute the material or use it for any profit-making activity or commercial gain

If the publication is distributed under the terms of Article $25 \mathrm{fa}$ of the Dutch Copyright Act, indicated by the "Taverne" license above, 


\section{UNDERSTANDING ADHERENCE}

Exploring the structural and psychosocial determinants of antiretroviral therapy adherence among pregnant women in Mpumalanga Province, South Africa

\section{SHANDIR RAMLAGAN}




\section{UNDERSTANDING ART ADHERENCE:}

EXPLORING THE STRUCTURAL AND PSYCHOSOCIAL DETERMINANTS OF ANTIRETROVIRAL THERAPY ADHERENCE AMONG PREGNANT WOMEN IN Mpumalanga Province, SOUth Africa

Shandir Ramlagan 
ISBN: 978-94-6380-601-5

Lay-out and Printing: Proefschriftmaken | Proefschriftmaken.nl

Cover Design: Ilze Visagie | www.hsrc.ac.za

(C) Copyright Shandir Ramlagan, Maastricht University, 2019

All rights reserved. No part of this publication may be reproduced, stored in a retrieval system or transmitted, in any form or by any means, electronic, mechanical, photocopying, recording or otherwise, without prior permission of the author or the copyright-owning journals for previous published chapters. 


\title{
UNDERSTANDING ART ADHERENCE:
}

\section{EXPLORING THE STRUCTURAL AND PSYCHOSOCIAL DETERMINANTS OF ANTIRETROVIRAL THERAPY ADHERENCE AMONG PREGNANT WOMEN IN Mpumalanga Province, SOUth Africa}

\author{
Thesis \\ To obtain the degree of Doctor at \\ Maastricht University, \\ on the authority of the \\ Rector Magnificus,
}

Prof.dr. Rianne M. Letschert

in accordance with the decision of the Board of Deans, to be defended in public on

Tuesday, $3^{\text {rd }}$ December 2019, at $12 \mathrm{~h} 45$

by

Shandir Ramlagan 


\section{Supervisors:}

Prof. dr. R.A.C. Ruiter;

Prof. dr. S. Sifunda, Human Sciences Research Council, South Africa;

Prof. dr. K. Peltzer, Human Sciences Research Council, South Africa

\section{Assessment Committee:}

Prof. dr. G.J. Kok, (chairman);

Prof. dr. B. van den Borne;

Prof. dr. M. de Bruin, Radboud UMC;

Prof. dr. J. Nijhuis;

Prof. dr. P. Reddy, Human Sciences Research Council, South Africa

The studies presented in this thesis was funded by the National Institutes of Health with grant number R01HD078187. 
To my late grandmother,

Tharawathie Ramlagun,

who wished for this doctorate more than anyone else 



\section{TABLE OF CONTENTS}

CHAPTER ONE

GENERAL INTRODUCTION

CHAPTER TWO

23

PREVALENCE AND FACTORS ASSOCIATED WITH FIXED-DOSE COMBINATION ANTIRETROVIRAL DRUGS ADHERENCE AMONG HIV POSITIVE PREGNANT WOMEN ON OPTION B TREATMENT IN MPUMALANGA PROVINCE, SOUTH AFRICA.

CHAPTER THREE

DETERMINANTS OF DISCLOSURE AND NON-DISCLOSURE OF HIV-POSITIVE STATUS, BY PREGNANT WOMEN IN RURAL SOUTH AFRICA

CHAPTER FOUR

CORRELATES OF PERCEIVED HIV-RELATED STIGMA AMONG HIV-POSITIVE PREGNANT WOMEN IN RURAL MPUMALANGA PROVINCE, SOUTH AFRICA

CHAPTER FIVE

75

SELF-REPORT AND DRY BLOOD SPOT MEASUREMENT OF ANTIRETROVIRAL MEDICATIONS AS MARKERS OF ADHERENCE IN PREGNANT WOMEN IN RURAL SOUTH AFRICA

CHAPTER SIX

SELF-REPORTED LONG-TERM ANTIRETROVIRALADHERENCE: A LONGITUDINAL STUDY AMONG HIV INFECTED PREGNANT WOMEN IN MPUMALANGA, SOUTH AFRICA

CHAPTER SEVEN

105

GENERAL DISCUSSION

REFERENCES

SUMMARY

VALORISATION

ACKNOWLEDGEMENTS 


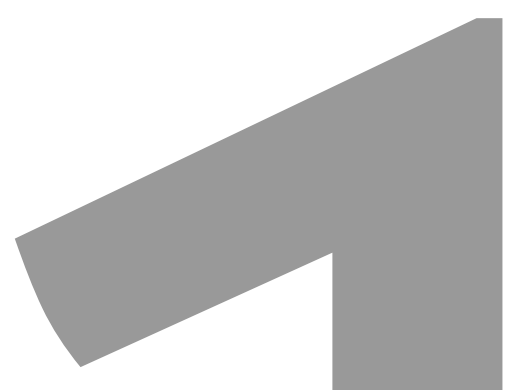

CHAPTER 1 
GENERAL INTRODUCTION 


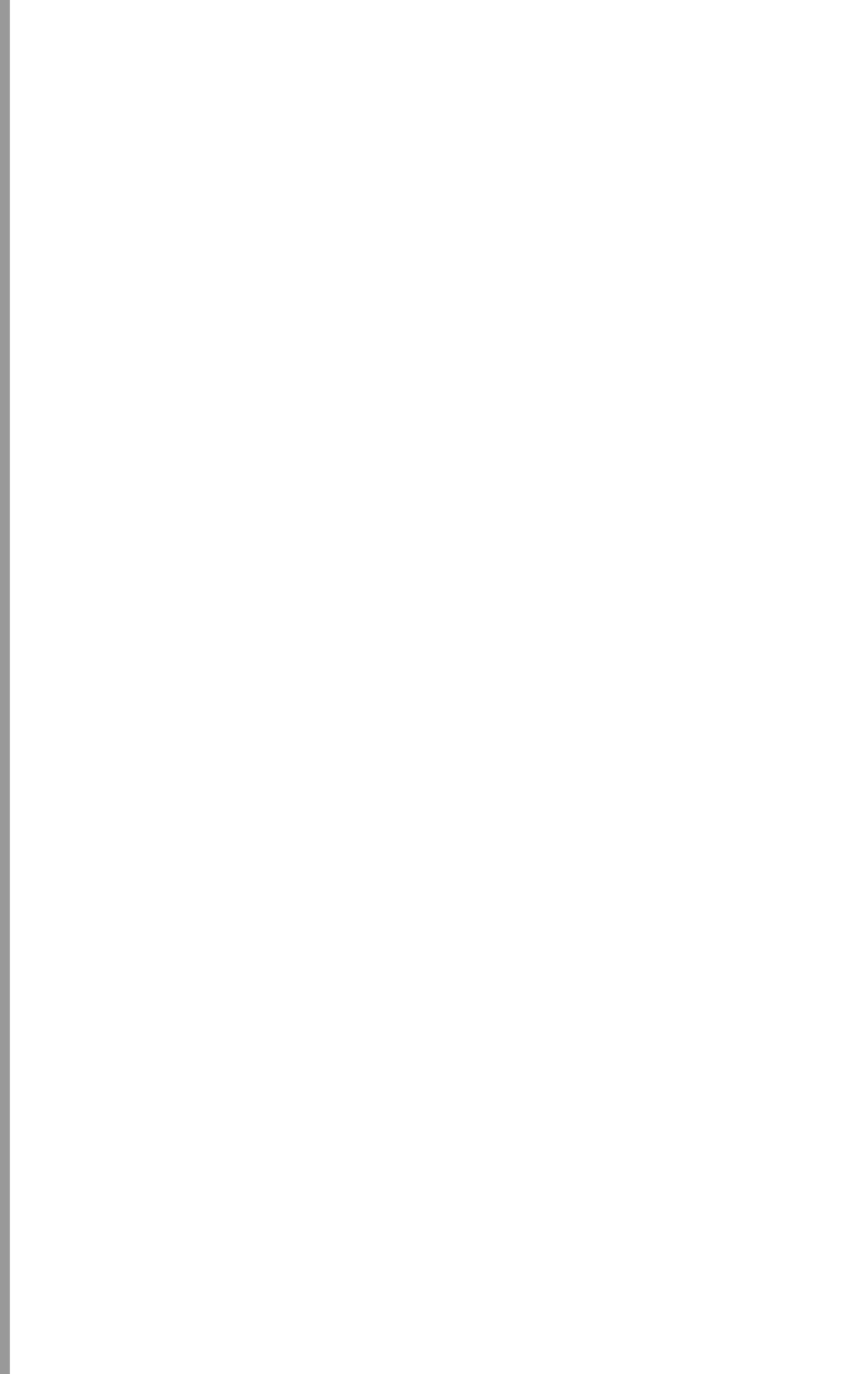




\section{Introduction}

Walking a mile in someone else's shoes is a phrase often used to state that one should not judge another until you experience what they experience on a daily basis. The question I had when I started my studies is why people do not take their antiretroviral therapy (ART) medication when the medication is readily available and especially when it is given to people who require it, free of charge. This question has perplexed me as I have seen my father not take his chronic medication for his heart condition and then later on seeing my partner not complete her course of prescribed antibiotics when she had a bacterial infection. This seemingly simple question led me to this doctoral study.

When trying to understand how to answer this question, I sought a population group that I was sure would take their medication and that would be relatively easy to convince to take their medication. I looked at women that were tested positive with the human immunodeficiency virus (HIV) and that were about to have a baby. I looked at pregnant women. Surly they would take the medication to protect their unborn child; surly they would take their medication so that they can be available for their child; surly they would want to grow old with their child. Women living with HIV are particularly vulnerable (Matseke, Rodriguez, Peltzer, \& Jones, 2016; Sangaramoorthy, Jamison, \& Dyer, 2017; Yuh, Ellwanger, Potts, \& Ssenyonga, 2014), and particularly those from low resource countries (Avert, 2018b; Bos, Schaalma, \& Pryor, 2008; Tsai, 2015).

This thesis investigates the contributing factors that facilitate ART adherence as well as non-adherence among HIV positive, pregnant women in Mpumalanga Province, South African. This was achieved by firstly understanding who these women were and then exploring the determinants of ART adherence by utilizing two selfreported ART adherence scales. The thesis then goes on to explore internalized stigma experienced by these women before going on to investigate disclosure of their HIV status and its impact on ART adherence. The thesis continues to explore the reliability of two self-reported ART adherence scales compared to adherence measured via dry blood spot (DBS) before reporting on the evaluation study of an ART adherence intervention given to these women from their first trimester of pregnancy until the baby was 12 months old and explores the change to adherence, change to non-adherence and stable adherence over time. The final chapter of this thesis brings together all five empirical studies and discusses them before concluding with recommendations.

This chapter initiates this thesis by firstly explaining the reason it was carried out (as done above) before going on to provide a background of prevalence of the HIV epidemic from a global perspective, providing a context of HIV in South Africa and finally in Mpumalanga. In providing this background, the HIV context of Nkangala 
and Gert Sibande municipal districts - the two study sites - are indicated. The chapter then describes the fight against HIV and thus the use of ART in this fight within the South African context. Next the chapter summarizes the international literature on the determinants of adherence to ART especially among pregnant women before concluding by introducing the five empirical studies that make up this thesis. Given the nature of the PhD thesis, that is a bundling of empirical studies that have been independently published, it should be expected that there will be much repetition between this chapter and the subsequent chapters of this thesis.

\section{The HIV context}

HIV or acquired immunodeficiency virus (AIDS) has become the greatest threat to human wellbeing and public health in this modern time. Globally, an estimated 36.9 million people in 2017 were living with HIV/AIDS, 1.8 million were newly infected with HIV, and 940000 died due to AIDS (UNAIDS, 2018). The region that is most affected by HIV/AIDS is Eastern and Southern Africa where 19.6 million people are living with HIV/AIDS, 800000 were newly infected with HIV, and 380000 died due to AIDS in 2017 (UNAIDS, 2018). Of great concern is that in terms of new HIV infections, approximately 66\% occur in Sub-Saharan Africa (UNAIDS, 2018).

In South Africa, in 2018, the population was estimated at approximately 58 million people with $51 \%$ being female (StatsSA, 2018). The overall HIV prevalence in South Africa, in 2017, was $14.0 \%$ or approximately 7.9 million people among all age groups (Simbayi, Zuma, Zungu, Moyo, Marinda, Jooste, Mabaso, Ramlagan, North, van Zyl, Mohlabane, Dietrich, Naidoo, \& Team., 2019). Among the reproductive age group, i.e. those aged 15 to 49 years old, $20.6 \%$ were living with HIV and specifically among females in this age group, HIV prevalence was an astoundingly high 26.3\% (Simbayi et al., 2019). In terms of possible mother to child transmission (MTCT) of HIV in South Africa, among infants, i.e. less than 12 months old, HIV prevalence in 2017 was $2.7 \%$ (Simbayi et al., 2019). When trying to identify mother and child pairs, in this national study, in the 0-2 years old age category, 415 mother and child pairs were identified where $9.8 \%$ of HIV-positive mothers had a child who was also HIV positive (Simbayi et al., 2019). Other data reports MTCT rates in South Africa of $1.5 \%$ at 6 weeks and $4.3 \%$ at 18 months (SANAC, 2016).

Within South Africa, there are 9 provinces all of which have different HIV prevalence rates (SANAC, 2016; Simbayi et al., 2019). The province of Mpumalanga hosts $8 \%$ or 4.5 million of South Africa's population (StatsSA, 2018) where $88 \%$ of the population relies on the public health sector (MPAC, 2017). The province as a whole is regarded as South Africa's third most rural province (MPAC, 2017) where the majority of the people in the province reside in a rural area (StatsSA, 2001). Poverty is more pronounced in rural areas where the area is marked with increased migration, dwindling opportunities, as well as structural and socio-economic depravations (Mpondo, 2018). As such, services available in rural areas tend to be poorer then in 
urban areas and thus the impact of HIV on rural homes is much greater (UNAIDS, n.d.). In South Africa, significant progress has been achieved in the implementation of prevention of mother to child transmission (PMTCT) of HIV programs. However, these improvements have, for the most part, occurred in urbanized areas, with rural areas remaining at unacceptably high levels of MTCT of HIV (Wettstein, Mugglin, Egger, Blaser, Salazar, Estill, Bender, Davies, \& Wandeler, 2012). Pregnancy outcomes is another such area where it is shown that woman in rural areas were more likely to have adverse pregnancy outcomes (Rollins, Coovadia, Bland, Coutsoudis, Bennish, Patel, \& Newell, 2007).

Mpumalanga province, in 2017, had the second highest HIV prevalence in the country at $17.3 \%$ (Simbayi et al., 2019). In terms of pregnant women, 15-49 years old, HIV prevalence was $36 \%$ in 2014 (MPAC, 2017). HIV/AIDS was reported as the second leading cause of death for males and females between the ages 5 to 14 year old in Mpumalanga and the leading cause of death for females 15-25 years old (Health Systems Trust, 2018). In terms of infants around 10 weeks old, during 2016/2017, 1.7\% in Mpumalanga province tested HIV positive on the polymerase chain reaction (PCR) test and within Nkangala district, 2.1\% tested HIV positive (Health Systems Trust, 2018). It is extremely important to note here that the South African target for infant HIV PCR test positive around 10 weeks is $1.4 \%$ and thus the figures reported above are extremely high and of great concern. The reported HIV prevalence in Gert Sibande district was $22.9 \%$ in 2017 (Simbayi et al., 2019) and is noted by the province itself as unacceptably high (MPAC, 2017). In Nkangala district, in 2014, HIV prevalence and was reported as $29.9 \%$ (MPAC, 2017), and between the years 2010-2015, HIV/AIDS was the fourth and fifth leading cause of death for under five year old females and males respectively (Health Systems Trust, 2018).

\section{The fight against HIV/AIDS}

In the fight against the HIV/AIDS epidemic, every country has both moral and medical obligation to fight through further prevention strategies and to decrease the incidence of HIV. For those infected already, provision of treatment, including antiretroviral therapy (ART) has become extremely important. In South Africa, the implementation of the Government's Comprehensive HIV/AIDS Care, Management and Treatment Plan started in the first quarter of 2004 (Johnson, 2012). ART is a proven and widely accepted treatment that is prescribed to HIV positive persons to suppress the virus and thus increase longevity (Kuhn, Semrau, Ramachandran, Sinkala, Scott, Kasonde, Mwiya, Kankasa ,Decker., Thea, \& Aldrovandi, 2009; Lohse, Hansen, Gerstoft, \& Obel, 2007). With ART, HIV can be classified as a chronic disease (Deeks, Lewin, \& Havlir, 2013) where those infected can live until their normal life expectancy but only with proper adherence to ART.

However, successes in ART lie largely on adherence to treatment protocols, since default and patient incompliance and non-adherence might lead to treatment 
failure and in some instances cause the HIV strain to be resistant (Wahl \& Nowak, 2000). This finding is similar to the extensive work done on antibiotic resistance where resistance in the infecting bacterial organism can also be associated with treatment failure, prolonged or additional hospitalization, increased cost of care, and increased mortality (French, 2005). Once resistance has occurred, antiviral agents are unable to eradicate infection hence prolonged clinical benefit requires the most continuous and sustained suppression of viral replication (Cingolani, Antinori, Rizzo, Murri, Ammassari, Baldini, Di Giambenedetto, Cauda, \& De Luca, 2002). Therefore, one of the critical obstacles to successful drug therapy can be caused by the imperfect adherence to a prescribed regimen (Wahl \& Nowak, 2000). The roll-out of ART in resource-poor settings, such as through the public health sector in African countries, has been cloaked in debate about resource allocation and the need for prioritization between prevention and treatment programs. The traditional objections to the public provision of ART have followed the arguments of the high cost of antiretroviral medications and the lack of appropriate infrastructure capable of delivering the therapy in poor countries (Berwick, 2002). However, as financial obstacles have begun to unravel, the potential for widespread provision of ART to HIV-infected people living in endemic countries has become possible, yet the debate continues to be marred by concerns over the feasibility of the public health systems of poor countries to manage the delivery of ART, and the potential public health threat of the development of multidrug-resistant strains of HIV arising from poor adherence to HAART regimen (Mehta, Moore, \& Graham, 1997).

Rollout of South Africa's public sector ART program, as mentioned above, began in 2004 (Johnson, 2012). To date, there were three main ART preventative treatment plans in place in South Africa to discourage HIV disease progression and transmission. These ART preventative treatment plans include Option A, Option B, and Option B+ (Bateman, 2013; Besada, Van Cutsem, Goemaere, Ford, Bygrave, \& Lynch, 2017; Schouten, Jahn, Chimbwandira, Harries, \& Damme, 2013). Each of the three above mentioned ART treatment option comprises of different inclusion criteria, timelines as well as frequency for the distribution of ART for both mother and infant. Options $B$ and $B+$ eliminated the need for CD4 counts prior to initialization on ART and are thus known as the test and treat models (UNICEF, 2012; WHO, 2010). In the Option $B$ treatment protocol, ART will continue to be provided to the mother after the cessation of breastfeeding only if the mothers' health requires it, whereas in Option B+ ART will continue for life (Besada et al., 2017). Option B+ model of treatment is regarded as best practice yet in resource limited locations, the compromised Option A or B is implemented. This thesis focused on those undergoing Option B treatment as it was the only available treatment in Mpumalanga public sector clinics during the recruitment phase of this study.

\section{ART Adherence}

Adherence relates to the extent to which the patient follows a prescribed regimen 
(Mehta, Moore, \& Graham, 1997). Basically it means (1) taking doses of drugs and sticking to the treatment regimen exactly as prescribed, (2) taking the correct dose of drugs, at the correct time, and in the correct way (for example, with the right type of food or fluid, and before or after a meal), and (3) looking after drugs to make sure that they are effective and safe to use (International HIV/AIDS Alliance, 2005). Poor adherence in general to any pharmacological treatment is viewed as restraining effective treatment of many health conditions, including tuberculosis, diabetes, high blood pressure, and mental illness to name a few (Robbins, Spector, Mellins, \& Remien, 2014).

The importance of adherence to ART for HIV has been well documented in the research literature (Chesney, Ickovics, Chambers, Gifford, Neidig, \& Zwickl, 2000; Giordano, Guzman, Clark, Charlebois, \& Bangsberg, 2004; Hecht, Wang, Collier, Little, Markowitz, Margolick, Kilby, Daar, Conway, \& Holte, 2006; Kastrissios, Suárez, Katzenstein, Girard, Sheiner, \& Blaschke, 1998). ART has the ability to lower ones virus level to a point where the virus becomes undetectable in the body. As such, it has been documented that women who are adherent are less likely to pass HIV on to their baby (Mellins, Chu, Malee, Allison, Smith, Harris, Higgins, Zorrilla, Landesman, Serchuck, \& Larussa, 2008; Williams, Wood, Dukay, Delva, Ginsburg, Hargrove, Stander, Sheneberger, Montaner, \& Welte, 2011). A very high level of adherence, at least $95 \%$, is necessary for ART to work effectively as ART has been reported unable to sustain complete viral suppression at less than 95\% adherence (van Servellen, Johiro, \& Tichacek, 2002) and can be associated with the development of viral resistance to medications (Wahl \& Nowak, 2000). Further, cross-resistance may develop across an entire class of medications, resulting in persons with HIV having viruses that are resistant to specific medications that those people have never taken (Avert, 2019).

Factors associated with adherence to ART can be grouped into four main groups including (1) patient factors and personality characteristics; (2) medication characteristics; (3) interpersonal characteristics; and (4) the general system within which care is administered (Chesney et al., 2000; Remien, 1998). Although current HIV treatments are extremely effective at reducing viral load for many, a host of aversive side effects, including nausea, diarrhea, headache, rash, vomiting, and sleep disturbance to name a few, may accompany ART (Catz, Kelly, Bogart, Benotsch, \& McAuliffe, 2000; Max \& Sherer, 2000; Watson, 2019). Whereas some side effects may subside with time, others persist as long as the medication is continued (Watson, 2019). These side effects may interfere with adherence because they can interfere with daily routines, obligations, and quality of life (Catz et al., 2000).

There are also growing concerns about long-term toxicities associated with prolonged use of ART (Max \& Sherer, 2000) which makes adherence to medication difficult as the concerns about taking the medication outweigh the necessity to take 
it (Horne, Weinman, \& Hankins, 1999). The necessity concerns framework is utilized to understand peoples adherence to medication and postulates that if a person's necessity to take the medication, in this case ART, outweighs their concerns about the medication, then the probability of the person taking the medication increases but if the concerns about the medication outweigh the necessity, then the probability of the person taking the medication drops (Horne, Buick, Fisher, Leake, Cooper, \& Weinman, 2004; Horne, Chapman, Parham, Freemantle, Forbes, \& Cooper, 2013; Horne, et al., 1999; Phillips, Diefenbach, Kronish, Negron, \& Horowitz, 2014).

Although many have benefited from improved and simpler dosing schedules, this evolution toward treatment simplicity does not necessarily lead to ART adherence. Thus, increased understanding of the complex dynamics associated with adherence to ART remains important. The complex dynamics include sociodemographic and psychosocial factors that are associated with ART adherence include age, income, knowledge, stigma, depression, and disclosure of HIV status to name a few (Heestermans, Browne, Aitken, Vervoort, \& Klipstein-Grobusch, 2016; Nachega, Uthman, Anderson, Peltzer, Wampold, Cotton, Mills, Ho, Stringer, McIntyre, \& Mofenson, 2012; Reda \& Biadgilign, 2012). Among HIV positive pregnant women from around the world, facilitators for better adherence to ART include higher education, higher income, PMTCT knowledge, previous PMTCT, HIV status disclosure, partner support, frequent ante-natal care (ANC) attendance, support groups, and being on lifelong ART (Nachega et al., 2012). Barriers to ART adherence include being of a younger age, single, substance use, depression, long duration of ART, higher viral load, and having AIDS (Nachega et al., 2012).

The side-effects and possible toxicity of ART, as mentioned above, coupled with the hormonal changes during pregnancy and its related discomforts including nausea, constipation, tenderness, headaches (Davis, 1996) could lead to non-adherence (Weiser, Tuller, Frongillo, Senkungu, Mukiibi, \& Bangsberg, 2010). Though the maternal desire to protect the unborn baby is a strong motivator for good adherence (Ekama, Herbertson, Addeh, Gab-Okafor, Onwujekwe, Tayo, \& Ezechi, 2012). Pregnant women who received basic PMTCT services and information, and who understood the importance of the viral load and CD4 test results exhibited improved emotional outlooks and were thus more apt to take actions to protect their unborn child (Futterman, Shea, Besser, Stafford, Desmond, Comulada, \& Greco, 2010).

In South Africa, especially among women, the social stigma of being infected with HIV is a common reason of sub-optimal adherence (Roberts \& Mann, 2000). Stigma associated with HIV transmission mode, including multiple sex partners, homosexual sex, and injected drug use, makes managing HIV disease different from managing other medical conditions and can directly interfere with health care utilization and adherence to care (Chesney et al., 2000). At the intersection of womanhood and 
poverty, HIV positive women's vulnerability to experiencing stigma is also shaped by societal disapproval of mothering in context of poverty and/or risk of transmitting HIV to a child (Sandelowski, Lambe, \& Barroso, 2004).

Disclosure of HIV serostatus has been emphasized as a crucial goal in HIV testing and counselling as well as PMTCT protocols (Alemayehu, Yohannes, Damte, Fantahun, Gebrekirstos, Tsegay, Goldberger, \& Yebyo, 2015; UNAIDS, 2011; WHO, 2012a). Reasons for disclosure include to adopt safer sexual behaviours, to prevent re-infection, to minimize the risk of HIV transmission to the unborn baby during pregnancy, at delivery and during breastfeeding (Shiyoleni, 2013). Disclosure of their HIV status by women to their male partner was found to significantly improve ART adherence during pregnancy in Tanzania (Kirsten et al., 2011) and Nigeria (Ekama et al., 2012). There were also significant increases to adherence to the PMTCT protocol in the post-natal period, such as returning for post counseling, increased adherence to ART medication, and increased condom use (Patel, Ratner, Gore-Felton, Kadzirange, Woelk, \& Katzenstein, 2012; Ramirez-Ferrero \& LustiNarasimhan, 2012), as well as lower levels of depression (Patel et al., 2012). In South Africa, disclosure and male partner involvement have similarly been shown to improve antiretroviral adherence during pregnancy among HIV positive women (Peltzer, Sikwane, \& Majaja, 2011). Lack of HIV status disclosure though has been identified as one of the major barriers to PMTCT effectiveness in rural areas (Bajunirwe \& Muzoora, 2005; Bancheno, Mwanyumba, \& Mareverwa, 2010; Jones, Peltzer, Weiss, Sifunda, Dwane, Ramlagan, Cook, Matseke, Maduna, \& Spence, 2014; Mepham, Zondi, Mbuyazi, Mkhwanazi, \& Newell, 2011; Rollins et al., 2007; Tabana, Doherty, Swanevelder, Lombard, Jackson, Zembe, \& Naik, 2012) as well as posing as a challenge to implementation of PMTCT programs in overburdened clinics (Hardon, Vernooij, Bongololo-Mbera, Cherutich, Desclaux, Kyaddondo, Ky-Zerbo, Neuman, Wanyenze, \& Obermeyer, 2012; Kuonza, Tint, Harris, \& Nabukenya, 2011; Turan, Bukusi, Onono, Holzemer, Miller, \& Cohen, 2011; Turan, Hatcher, MedemaWijnveen, Onono, Miller, Bukusi, \& Turan, 2012).

\section{This Thesis}

Although much is known about the medical complexities of ART and thus nonadherence to the regime, there is limited data available on the social aspects of ART adherence during pregnancy (Nachega et al., 2012) and no data available on Option B and pregnancy. Non-adherence to ART is a multifaceted, complex problem that may be exacerbated by social and systemic factors related to issues such as gender, geographical location (urban/rural), socioeconomic status, emotional wellbeing and health literacy, while all further complicated by stigma (Holzemer \& Uys, 2004; Skinner \& Mfecane, 2004). As such it is difficult for clinicians to assess patient willingness to adhere to ART. 
This thesis presents both exploratory and descriptive studies to better understand adherence as well as non-adherence to ART medication among HIV positive pregnant women in Mpumalanga province, South Africa. It accomplishes this by utilising data from a single longitudinal study and as such, the majority of chapters are based on the same dataset.

Chapter 2 of this study explores the prevalence and factors associated with fixeddose combination ARV drugs adherence among HIV positive pregnant women on Option B treatment in Mpumalanga province, South Africa. This study is important as it sets the scene in understanding the population group of this study and more importantly, it utilizes two different ART adherence scales to explore demographic, socioeconomic, health, and behavioral correlates of ARV non-adherence.

Chapter 3 examines the determinants of disclosure and non-disclosure of the pregnant woman's HIV-positive status to either someone or to their partner. Disclosure of ones HIV positive status remains one of the major ART adherence interventions and understanding reasons for disclosure may assist in encouraging HIV positive pregnant women to disclose their status. ART adherence was used as a covariate to understand its association with disclosing ones HIV positive status to someone or to one's partner.

Chapter 4 explores the correlates of perceived internalized stigma utilizing a 40item internalized stigma scale. The study not only validated the scale for use in South Africa, but also assessed the socioeconomic, behavioral and HIV-related correlates of internalized stigma factors of personalized stigma (17 items), concerns with public attitudes about people with HIV ( 7 items), disclosure concerns ( 6 items), and negative self-image (10 items) among HIV positive pregnant women. In this study, ART adherence was used as a covariate to understand the association of ART adherence with each of the four internalized stigma subscales. Although published, the version of this study that appears in this thesis is an extended version which includes a more in-depth literature review as well as extra analysis showing an extra step in the factor analysis.

Chapter 5 presents findings from comparing two self-report adherence scales, namely the visual analog scale (VAS) (Giordano et al., 2004) and the Adult AIDS Clinical Trials Group (AACTG) (Chesney et al., 2000) scale, with the participants ARV dried blood spots (DBS) adherence measure. Participants completed a selfadministered questionnaire at 32 weeks pregnancy and then provided five drops of their blood. The study compares the adherence rates among the three measures.

Chapter 6 examines the self-reported long-term ART adherence among the HIV positive pregnant women in Mpumalanga province, South Africa. The study included participants that completed both baseline assessments as well as the final 
assessment 12 months after birth of the child. In this study, change in adherence over time was investigated. Reasons for missing ART are also reported.

Chapter 7 provides a general discussion of the empirical findings presented in chapter's two to six of this thesis. The chapter also reflects on the methodology used in this study as well as practical implications of the thesis. 


\section{CHAPTER 2}




\section{PREVALENCE AND FACTORS ASSOCIATED WITH FIXED-DOSE COMBINATION ANTIRETROVIRAL DRUGS ADHERENCE AMONG HIV POSITIVE PREGNANT WOMEN ON OPTION B TREATMENT in MPUMALANGA PROVINCE, SOUTH AFrica}

Published as:

Ramlagan, S., Peltzer, K., Ruiter, R.A.C., Barylski, N.A., Weiss, S.M., \& Sifunda, S. (2018). Prevalence and factors associated with fixed-dose combination antiretroviral drugs adherence among HIV positive pregnant women on Option B treatment in Mpumalanga province, South Africa. 


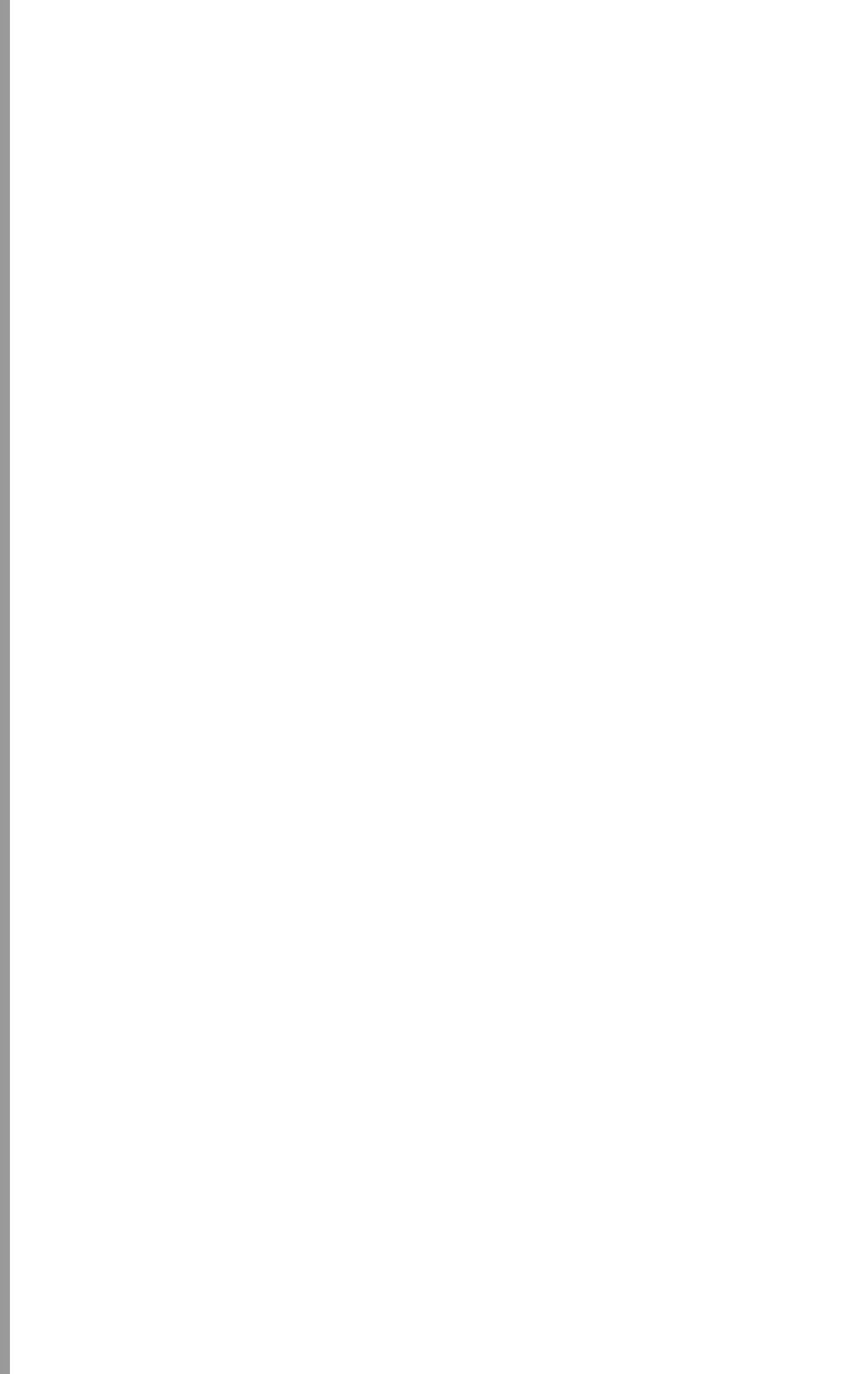




\section{Introduction}

Globally an estimated 36.7 million people were living with HIV in the year 2016; 1.8 million became newly infected and 1 million lost their lives to AIDS-related diseases (UNAIDS, 2017b). Sub-Saharan Africa remains the global epicenter of this epidemic where nearly $71 \%$ of the global HIV infections occurred in 2014 with an estimated 24.7 million people (UNAIDS, 2014). The latest South African populationbased HIV prevalence and incidence study estimated HIV prevalence at $12.2 \%$ or 6.4 million people, and HIV incidence at $1.1 \%$ or 469,000 people (Shisana, Rehle, Simbay, Zuma, Jooste, Zungu, Labadarios, \& Onoya, 2014). Because of the high incidence and prevalence of HIV in South Africa, antiretroviral (ARV) adherence of HIV-infected pregnant women on fixed dose combinations (FDC) represents an important prevention strategy.

South Africa has approximately 3.7 million HIV-positive people on ARV treatment (SANAC, 2016), which is more than any other country in the world (UNAIDS, 2017b). Each year, over 1.4 million HIV infected women give birth, with $91 \%$ residing in subSaharan Africa (WHO/UNAIDS/UNICEF, 2010), leading to a high potential rate of mother-to-child transmission (MTCT) of HIV. Currently, MTCT rates in South Africa are $1.5 \%$ at 6 weeks and $4.3 \%$ at 18 months (SANAC, 2016). In order to reduce MTCT rates, pregnant women need to adhere to their ARVs, as women who are adherent are less likely to transmit their HIV to their baby (Mellins et al., 2008; Williams et al., 2011). There were two ARV treatment plans in place in South Africa during 2014-2015 and include Option A and Option B. This was due to the phasing out of Option A for the better Option B. Options B is known as the test and treat model as the protocol states that as soon as an individual tests HIV-positive, they can be placed on ARV treatment immediately while they wait for their CD4 count (UNICEF, 2012; WHO, 2012b). This is important because, in Option A, ARV treatment could only begin once a CD4 count result is obtained. In a resource poor setting like Mpumalanga Province, South Africa, this could take many months. In the Option $B$ treatment protocol, ARVs will continue to be provided to the mother after the cessation of breastfeeding only if the mothers' health requires it.

An important setback, as noted in Malawi, was that those who initiated their ARV regimen during pregnancy were twice as likely to miss their first follow-up visit (Tenthani, Haas, Tweya, Jahn, Van Oosterhout, Chimbwandira, Chirwa, Ng'Ambi, Bakali, Phiri, Myer, Valeri, Zwahlen, Wandeler, \& Keiser, 2014). These missed appointments and follow-ups decrease the effectiveness of the treatment (Nachega et al., 2011; Tenthani et al., 2014). Another factor to impact ARV adherence negatively is alcohol abuse (Kreitchmann et al., 2012; Mellins et al., 2008). It would seem that as alcohol intake increases, people become less adherent. Furthermore, a large number of pregnant women do not want to deal with both the side effects of the ARVs and the normal side effects of pregnancy at once, so many interrupt 
treatment or postpone initiation of it to avoid side effects (Weiser et al., 2010) such as exacerbated nausea, vomiting, and gastro-intestinal issues, to state a few (Nachega et al., 2012). In early pregnancy, $70-85 \%$ of women experience morning sickness, and in later pregnancy many experience heartburn (Nachega et al., 2012). To avoid exacerbation of these symptoms by ARV medications, adherence during this period can be reduced (Mepham et al., 2011; Nachega et al., 2012). Finally, the lack or presence of social support greatly affects the way a woman, especially a pregnant women, follows her HIV treatment plan (Nachega et al., 2012). In South Africa especially, the social stigma of being infected with HIV is a common reason of sub-optimal adherence (Mepham et al., 2011; Roberts \& Mann, 2000; Simbayi et al., 2007). On the other hand, disclosure of HIV status and treatment support by a partner has been shown to be associated with good adherence (Ekama et al., 2012). Those who receive proper social support tend to have reduced depression scores and better ARV adherence (Futterman et al., 2000).

However, adherence can also be increased as a result of the pregnancy, as maternal desire to protect the unborn child is one of the greatest motivators of good adherence (Ekama et al., 2012). Factors associated with better adherence include strong beliefs about the necessity of medication (Chesney et al., 2000; Horne et al., 2004) as well as older age where the older the person is, the more likely it is that the person will adhere to the ARVs (Kreitchmann et al., 2012; Nachega et al., 2012). When women understood the importance of the viral load and CD4 test results and received basic prevention of mother-to-child transmission (PMTCT) services, their emotional outlooks improved and they were more apt to take actions to protect their unborn child (Futterman et al., 2010).

There is extremely limited data on ARV adherence during pregnancy (Nachega et al., 2012). In terms of the Option B regime and pregnant women, there are no data available. The purpose of this study was to examine the prevalence and factors associated with fixed-dose combination antiretroviral drugs among HIV-infected pregnant women on Option B treatment in South Africa.

\section{Materials and Methods}

\section{Sample and Procedure}

Cross-sectional data of 673 HIV-positive pregnant women were collected over a 12-month period from April 2014 to March 2015. This baseline data is part of the Protect Your Family randomised controlled trial (Jones et al., 2014) conducted in 12 community health centres (CHCs) in Gert Sibande and Nkangala districts in Mpumalanga province, South Africa. The participants were all less than six months pregnant at the time of the baseline interview, and were attending antenatal care (ANC) at the study CHCs. During the baseline period, all HIV-positive pregnant women in Mpumalanga province attending public ANC were offered Option B 
treatment. As per the South African PMTCT protocol (Department of Health, 2014) and the South African antiretroviral treatment guidelines (Department of Health, 2013), pregnant women recruited during our study period, were provided tenofovir (TDF) + emtricitabine (FTC) or lamivudine (3TC) + efavirenz (EFV) as fixed dose combinations.

Potential participants were referred to trained study fieldworkers by ANC nurses at the study $\mathrm{CHCs}$ utilising the indicators of less than six months pregnant and HIV-positive. Fieldworkers screened the potential participant adding the following indicators: (1) being 18 years and older, (2) having a current partner (no necessarily the father of the baby), and (3) having not been recruited in this study at another $\mathrm{CHC}$. If the participant met all screening indicators, they were invited to participate and, if interested, the participants were then introduced to the study information sheet and consent form in English, isizulu, or seSotho. Once the consent process was completed, participants completed the baseline assessment utilising the Audio Computer-Assisted Self-Interview (ACASI) technology(NIMH Multisite HIV/ STD Prevention Trial for African American Couples Group, 2008), which was used to increase confidentiality and self-disclosure and ensure the inclusion of women across all literacy levels.

The ACASI software was loaded onto touch screen Lenovo ThinkPad X230 tablet/ laptop (with $180^{\circ}$ swivel screen). Logitech h150 stereo over-ear headphones were utilised to maintain privacy. All participants were given brief training on the usage of the laptop touchscreen, headphones, and ACASI software. The fieldworkers assisted the participants to complete the initial demographic variables to ensure they were comfortable with ACASI, and all other questions were then completed individually by the participant with the fieldworker in the room to assist should a question or problem arise and to collect the completed questionnaire once the participant had completed it. Participants were compensated with R50.00 (South African Rand = US\$4.72) for time and transportation.

\section{Measures}

TheACASI questionnaire requested information on sociodemographic characteristics, HIV related issues, intimate partner violence, stigma, and depression. How these factors might impact adherence to ARVs was the main outcome measure.

\section{Socio-Demographic Characteristics}

Questions regarding socio-demographic characteristics included age at last birthday; language-Nguni (isiZulu, isiXhosa, isiSwati, and isiNdebele) and others (Afrikaans, English, Sesotho, Sepedi, Setswana, Tshivenda, Xitsonga, other European, and other); educational attainment (completed Grade 11 or less and completed Grade 12 or more); partner status (are couples living together or not); employment status (not employed versus employed, volunteer or student); income per month 
in South African Rand (ZAR) where the South African Government child grant was R310.00 (UD\$29.27) during the study period; number of children; if this pregnancy was planned or unplanned; and alcohol use of more than 2 drinks on at least one occasion in the previous four weeks (yes vs. no).

\section{HIV-Related Characteristics}

Questions regarding HIV-related characteristics asked participants for the following: whether HIV was diagnosed during their current pregnancy (yes vs. no); the period of time spent on ARV medication ( $<12$ months vs. $\geq 12$ months); whether the their HIV status was disclosed to anyone (yes vs. no); whether any of their children were HIV-positive (yes vs. no); whether their partner was HIV-positive (yes vs. no); and, in terms of male involvement, whether their male partner knew what happened in the antenatal clinic (yes vs. no). If the respondent had no children, they were not asked if any of their children were HIV-positive, and the question of partners' status was not asked if the respondent stated "no" or "don't know" to the question, "Has your partner or spouse been tested for HIV?"

HIV knowledge was also attained, and questions included HIV transmission, condom use, PMTCT, and AIDS, and was assessed using 18 items adapted from an AIDSrelated knowledge scale (Carey \& Schroder, 2002). HIV knowledge was scored for the number of correct responses (Yes or No), with Don't Know responses scored as incorrect (score 1 or 0 ). The possible range of scores 0 to 18 was expressed as the percentage correct, meaning that the higher the score, the more HIV knowledge a respondent had. The scores were dichotomized using a cutoff of greater than or less than and equal to $13 / 18$ correct (Wagenaar, Sullivan, \& Stephenson, 2012). Cronbach's Alpha reliability for the scale was $\alpha=0.74$.

\section{Intimate Partner Violence (IPV)}

An adapted 18 -item version of that Conflict Tactics Scale 18 (CTS-18) (Gelles \& Straus, 1979; Straus, 1979) was used to assess psychological and physical aggression, as well as reasoning. Respondents were presented with 18 conflict situations such as "discussed the issue calmly," "cried," "did something to spite you," "threw something at you," "slapped you," etc. and were asked to rate the number of times she and her partner may had engaged in such conflict in the previous month on a seven point scale of 0 (never) to 6 (more than 20 times). A higher score is indicative of increased IPV. Cronbach's Alpha reliability for the scale was $\alpha=0.84$.

\section{Aids-Related Stigma}

The nine-item AIDS-Related Stigma Scale (ARSS) (Kalichman, Simbayi, Jooste, Toefy, Cain, Cherry, \& Kagee, 2005) was used to assess externalised stigma using items such as "People who have AIDS are dirty," "People who have AIDS should be ashamed," etc. This is a dichotomous scale using a score of 0 (disagree) to 1 (agree). Due to internal reliability of the original nine-item scale, question four, a reverse 
scored item, was removed to increase reliability. Scores on the scale range from 0 to 8 , where higher scores indicate greater levels of stigma. Cronbach's Alpha reliability for the scale was $\alpha=0.73$.

\section{Depression}

The 10-item Edinburgh Postnatal Depression Scale 10 (EPDS-10) (Cox, Holden, \& Sagovsky, 1987) asked participants to rate how often they had experienced different symptoms associated with depression in the previous seven days. Questions included "I have been able to laugh and see the funny side of things," "I have felt sad or miserable," etc. Scores ranged from 0 to 30, where the higher the score, the more the likelihood of depression being experienced. This paper utilised the validated cut-off score for South African populations at score 12 (Lawrie, Hofmeyr, de Jager, \& Berk, 1998). Cronbach's Alpha reliability for the scale was $\alpha=0.66$.

\section{Adherence}

Two self-report instruments were used to assess adherence, namely the Adult AIDS Clinical Trials Group (AACTG) (Chesney et al., 2000), and an adapted version of the ARV adherence Visual Analog Scale (VAS) (Giordano et al., 2004). The AACTG adherence measure is a four day recall instrument asking "How many doses did you miss: (1) yesterday, (2) day before yesterday, (3) three days ago, and (4) four days ago." Given that the current ARV regiment in South Africa is one combination pill per day, a dose would translate to one ARV pill per day. Participants were recorded as adherent if they had taken all (100\%) of their ARV medication over the last four days. In order to understand non-adherence, the AACTG instrument provides 14 possible reasons a person may have for missing their medication and includes but is not limited to the following: were away from home, had too many pills to take, felt sick or ill, wanted to avoid side effects, and so on (Chesney et al., 2000). Responses were dichotomized to yes vs. no.

The second instrument utilised in this study was an adapted version of the VAS which included ARV usage over the previous seven days and asked participants if they had taken "None," "Half," or "All" of their medication in the previous seven days (Giordano et al., 2004). If participants reported that they had taken "All" of their medication in the previous seven days, they were recorded as "adherent" and coded as 1 in the statistical program. Participants were recorded as 0 if they too reported that they had taken "None" or "Half" of their medication on any day in the previous seven days.

\section{Data Analysis}

Software SPSS, version 24.0 (Statistical Product and Service Solutions, IBM, New York, NY, USA) was used for data analyses. Frequencies, means, and crosstabulations were calculated to describe the sample. Reliability tests were done on the ARSS, EPDS, and IPV scales. A $t$-test was conducted on adherence measures 
and self-rated adherence measure. Bivariate analyses and multivariable logistic regressions were used to investigate associations between the outcomes ARV adherence and socioeconomic, HIV-related, and behavioural variables. Associations were considered significant at $p<0.05$. All statistically significant variables in the bivariate analyses were included in the multivariable model. Multi-collinearity was tested.

\section{Ethical Approval}

Ethics approval for the study was granted by the HSRC Research Ethics Committee with Protocol No REC 4/21/08/13 as well as the University of Miami Miller School of Medicine Institutional Review Board (IRB ID: 20130238 (CR00006122)). Approval was also obtained from the Mpumalanga Provincial Government: Department of Health.

\section{Results}

A total of 673 HIV-positive pregnant women, with a mean age of 28 years old (SD = $5.73)$, were entered into this study. The majority (58\%) were between the ages of 18 and 29 years old. In terms of sample characteristics, Table 1 shows the majority (81\%) of respondents spoke an Nguni language, which includes Xhosa, Zulu, Swati and Ndebele, while only $29 \%$ had completed school. All participants in this study had a partner, as this was a study inclusion requirement, yet the majority (62\%) of respondents did not live with their partners. Unemployment was high, as $78 \%$ of respondents reported that they were unemployed with $33 \%$ of respondents receiving less than $\mathrm{R} 310$ monthly income. Only $21 \%$ or 139 respondents stated that they do not have other children with just over half (53\%) reported that this current pregnancy was unplanned. In terms of alcohol intake, $14 \%$ of our pregnant respondents reported drinking two or more alcoholic drinks on at least one occasion in the previous four weeks.

Table 1 also shows ARV adherence on the two adherence measures across sociodemographic variables. Overall, $21 \%$ of respondents reported to be nonadherent over the four-day recall AACTG scale, while 31\% reported to be nonadherent on the seven-day recall VAS. With all the sociodemographic variables, the VAS always reported $10 \%$ higher non-adherence of respondents when compared to the AACTG scale. In terms of those couples "not living together," $23 \%$ and $34 \%$ reported non-adherence with AACTG and VAS, respectively, and $19 \%$ and $27 \%$ of those "living together" reported non-adherence with AACTG and VAS, respectively. Twenty-five percent and $36 \%$ of respondents who receive a monthly income of less than R310 reported non-adherence with AACTG and VAS, respectively; $19 \%$ and $29 \%$ of those with a higher monthly income reported non-adherence with AACTG and VAS, respectively. Of those that stated that this was an unplanned pregnancy, $23 \%$ and $32 \%$ reported non-adherence with AACTG and VAS, respectively, while 
$20 \%$ and $30 \%$ of those who reported that this was a planned pregnancy reported non-adherence with AACTG and VAS, respectively. In this survey of HIV-positive pregnant women, $14 \%$ of respondents reported drinking two or more alcoholic drinks on at least one occasion in the previous four weeks. Of these, $39 \%$ on AACTG and $49 \%$ on VAS reported non-adherence.

Table 1: Sample characteristics.

\begin{tabular}{|c|c|c|c|c|c|}
\hline \multirow[t]{2}{*}{ Variable } & \multirow[t]{2}{*}{ Sample } & \multicolumn{2}{|c|}{ AACTG Adherence } & \multicolumn{2}{|c|}{ VAS Adherence } \\
\hline & & Adherent & $\begin{array}{l}\text { Non-Adherent } \\
\text { (Missed at Least } 1 \\
\text { Dose) }\end{array}$ & Adherent & $\begin{array}{l}\text { Non-Adherent } \\
\text { (Missed at Least } 1 \\
\text { Dose) }\end{array}$ \\
\hline Socio-demographics & $N(\%)$ & $N(\%)$ & $N(\%)$ & $N(\%)$ & $N(\%)$ \\
\hline All & $673(100)$ & $530(78.8)$ & $143(21.2)$ & $463(68.8)$ & $210(31.2)$ \\
\hline \multicolumn{6}{|l|}{ Language } \\
\hline Nguni languages & $546(81.1)$ & $432(79.1)$ & $114(20.9)$ & $373(68.3)$ & $173(31.7)$ \\
\hline Other languages & $127(18.9)$ & $98(77.2)$ & $29(22.8)$ & 90 (70.9) & $37(29.1)$ \\
\hline \multicolumn{6}{|l|}{ Educational attainment } \\
\hline Grade 11 and less & $481(71.5)$ & $386(80.2)$ & $95(19.8)$ & $340(70.7)$ & $141(29.3)$ \\
\hline Grade 12 or more & $192(28.5)$ & $144(75.0)$ & $48(25.0)$ & $123(64.1)$ & $69(35.9)$ \\
\hline \multicolumn{6}{|c|}{ Relationship status (couples) } \\
\hline Not living together & $419(62.3)$ & $324(77.3)$ & $95(22.7)$ & $277(66.1)$ & $142(33.9)$ \\
\hline Living together & $254(37.7)$ & $206(81.1)$ & $48(18.9)$ & $186(73.2)$ & $68(26.8)$ \\
\hline \multicolumn{6}{|l|}{ Employment status } \\
\hline \multirow{2}{*}{$\begin{array}{l}\text { Not employed } \\
\text { Employed, Volunteer } \\
\text { or Student }\end{array}$} & $527(78.3)$ & $416(78.9)$ & $111(21.1)$ & $363(68.9)$ & $164(31.1)$ \\
\hline & $146(21.7)$ & $114(78.1)$ & $32(21.9)$ & $100(68.5)$ & $46(31.5)$ \\
\hline \multicolumn{6}{|l|}{ Income (ZAR) per month } \\
\hline$<$ R310 & $221(32.8)$ & $165(74.7)$ & $56(25.3)$ & $141(63.8)$ & $80(36.2)$ \\
\hline R311 or more & $452(67.2)$ & $365(80.8)$ & $87(19.2)$ & $322(71.2)$ & $130(28.8)$ \\
\hline \multicolumn{6}{|l|}{ Number of children } \\
\hline None & $139(20.7)$ & $109(78.4)$ & $30(21.6)$ & $95(68.3)$ & $44(31.7)$ \\
\hline One or more & $534(79.3)$ & $421(78.8)$ & $113(21.2)$ & $368(68.9)$ & $166(31.1)$ \\
\hline \multicolumn{6}{|l|}{ Unplanned pregnancy } \\
\hline Yes & $356(52.9)$ & $276(77.5)$ & $80(22.5)$ & $242(68.0)$ & $114(32.0)$ \\
\hline No & $317(47.1)$ & $254(80.1)$ & $63(19.9)$ & $221(69.7)$ & $96(30.3)$ \\
\hline \multicolumn{6}{|c|}{ Alcohol use of more than 2 drinks on at least one occasion in the previous four weeks } \\
\hline No & $581(86.3)$ & $474(81.6)$ & $107(18.4)$ & $416(71.6)$ & $165(28.4)$ \\
\hline Yes & $92(13.7)$ & $56(60.9)$ & $36(39.1)$ & $47(51.1)$ & $45(48.9)$ \\
\hline
\end{tabular}

Table 2 shows that just over half (54\%) of all respondents reported that they were diagnosed with HIV during this pregnancy, and three quarters had initiated ARV medication less than 12 months prior. A quarter of respondents stated that they had an HIV-positive partner, and 58\% stated that their partner was involved in the pregnancy and are aware of what occurs during clinic visits (male involvement). In terms of disclosing their HIV status to anyone, $72 \%$ of respondents stated that they had disclosed their HIV status. Just over three-fifths demonstrated high knowledge of HIV and PMTCT issues. Of those respondents that have children (79\%), a low 
percentage (4.2\%) reported that they had an HIV-positive child. In terms of IPV, stigma, and depression, a high $20 \%$ of respondents reported experiencing IPV, $41 \%$ reporting stigma, and $49 \%$ reporting depression.

In terms of adherence, Table 2 shows that $23 \%$ and $33 \%$ of those respondents who had been on ARV medication for less than 12 months reported non-adherence with AACTG and VAS, respectively, while those $25 \%$ who had been on ARV medication for a year or longer reported $15 \%$ and $29 \%$ non-adherence with AACTG and VAS, respectively. Those who had disclosed their HIV status to anyone, $18 \%$ and $28 \%$ reported non-adherence with AACTG and VAS, respectively, while $29 \%$ and $40 \%$ of those who had not disclosed it reported non-adherence with AACTG and VAS, respectively. Twenty-seven percent and $39 \%$ of respondents that demonstrated no to low HIV knowledge reported non-adherence with AACTG and VAS, respectively, while $18 \%$ and $27 \%$ of those that demonstrated high HIV knowledge reported nonadherence with AACTG and VAS, respectively. In terms of IPV, of those respondents who stated that they experienced IPV, $28.8 \%$ and $37.9 \%$ reported non-adherence with AACTG and VAS, respectively, yet of those who reported not experiencing IPV, $19 \%$ and $30 \%$ reported non-adherence with AACTG and VAS, respectively. Of those who experienced internalised stigma, $25 \%$ and $36 \%$ reported non-adherence with AACTG and VAS, respectively, while $16 \%$ and $28 \%$ of respondents who reported no internalised stigma reported non-adherence with AACTG and VAS, respectively. In terms of respondents who reported higher depression scores, $27 \%$ and $37 \%$ reported non-adherence with AACTG and VAS, respectively, yet $16 \%$ and $26 \%$ of those with lower depression scores reported non-adherence with AACTG and VAS, respectively.

Table 2: Health and behaviour characteristics.

\begin{tabular}{|c|c|c|c|c|c|}
\hline \multirow[t]{3}{*}{ Variable } & \multirow[t]{2}{*}{ Sample } & \multicolumn{2}{|c|}{ AACTG Adherence } & \multicolumn{2}{|c|}{ VAS Adherence } \\
\hline & & Adherent & $\begin{array}{l}\text { Non-Adherent } \\
\text { (Missed at } \\
\text { Least } 1 \text { Day) }\end{array}$ & Adherent & $\begin{array}{l}\text { Non-Adherent } \\
\text { (Missed at Least } \\
1 \text { Day) }\end{array}$ \\
\hline & $N(\%)$ & $N(\%)$ & $N(\%)$ & $N(\%)$ & $N(\%)$ \\
\hline All & $673(100)$ & $530(78.8)$ & $143(21.2)$ & $463(68.8)$ & $210(31.2)$ \\
\hline \multicolumn{6}{|c|}{ Diagnosed with HIV in this pregnancy } \\
\hline No & $308(45.8)$ & $247(80.2)$ & $61(19.8)$ & $214(69.5)$ & $94(30.5)$ \\
\hline Yes & $365(54.2)$ & $283(77.5)$ & $82(22.5)$ & $249(68.2)$ & $116(31.8)$ \\
\hline \multicolumn{6}{|c|}{ Time on ARV medication } \\
\hline$<12$ months & $506(75.2)$ & $388(76.7)$ & $118(23.3)$ & $341(67.4)$ & $165(32.6)$ \\
\hline$\geq 12$ months & $167(24.8)$ & $142(85.0)$ & $25(15.0)$ & $122(73.1)$ & 45 (26.9) \\
\hline \multicolumn{6}{|c|}{ Disclosure of HIV serostatus to anyone } \\
\hline No & $188(27.9)$ & $133(70.7)$ & $55(29.3)$ & $113(60.1)$ & 75 (39.9) \\
\hline Yes & $485(72.1)$ & 397 (81.9) & $88(18.1)$ & $350(72.2)$ & $135(27.8)$ \\
\hline \multicolumn{6}{|l|}{ HIV-positive children } \\
\hline No or do not know & $506(94.8)$ & $398(78.7)$ & $108(21.3)$ & $351(69.4)$ & $155(30.6)$ \\
\hline Yes & $28(5.2)$ & $23(82.1)$ & 5 (17.9) & $17(60.7)$ & $11(39.3)$ \\
\hline
\end{tabular}


Table 2: Health and behaviour characteristics (cont...)

\begin{tabular}{|c|c|c|c|c|c|}
\hline \multirow[t]{3}{*}{ Variable } & \multirow[t]{2}{*}{ Sample } & \multicolumn{2}{|c|}{ AACTG Adherence } & \multicolumn{2}{|c|}{ VAS Adherence } \\
\hline & & Adherent & $\begin{array}{l}\text { Non-Adherent } \\
\text { (Missed at } \\
\text { Least } 1 \text { Day) }\end{array}$ & Adherent & \multirow{2}{*}{$\begin{array}{l}\text { Non-Adherent } \\
\text { (Missed at } \\
\text { Least } 1 \text { Day) } \\
N \text { (\%) }\end{array}$} \\
\hline & $N(\%)$ & $N(\%)$ & $N(\%)$ & $N(\%)$ & \\
\hline \multicolumn{6}{|l|}{ HIV-positive partner } \\
\hline No or do not know & $506(75.2)$ & $387(76.5)$ & $119(23.5)$ & $335(66.2)$ & $171(33.8)$ \\
\hline Yes & $167(24.8)$ & $143(85.6)$ & $24(14.4)$ & $128(76.6)$ & $39(23.4)$ \\
\hline \multicolumn{6}{|l|}{ Male involvement } \\
\hline No & $281(41.8)$ & $217(77.2)$ & $64(22.8)$ & $188(66.9)$ & $93(33.1)$ \\
\hline Yes & $392(58.2)$ & $313(79.8)$ & $79(20.2)$ & $275(70.2)$ & $117(29.8)$ \\
\hline \multicolumn{6}{|l|}{ HIV knowledge } \\
\hline No/Low & $257(38.2)$ & $187(72.8)$ & $70(27.2)$ & $158(61.5)$ & $99(38.5)$ \\
\hline High & $416(61.8)$ & $343(82.5)$ & $73(17.5)$ & $305(73.3)$ & $111(26.7)$ \\
\hline \multicolumn{6}{|l|}{ Intimate partner violence } \\
\hline $\begin{array}{l}\text { No mild or No severe } \\
\text { physical violence }\end{array}$ & $541(80.4)$ & $436(80.6)$ & $105(19.4)$ & $381(70.4)$ & $160(29.6)$ \\
\hline $\begin{array}{l}\text { Mild or severe physical } \\
\text { violence }\end{array}$ & $132(19.6)$ & $94(71.2)$ & $38(28.8)$ & $82(62.1)$ & $50(37.9)$ \\
\hline \multicolumn{6}{|l|}{ AIDS-Related Stigma } \\
\hline No & $400(59.4)$ & $326(81.5)$ & 74 (18.5) & $288(72.0)$ & $112(28.0)$ \\
\hline Yes & $273(40.6)$ & $204(74.7)$ & $69(25.3)$ & $175(64.1)$ & $98(35.9)$ \\
\hline \multicolumn{6}{|l|}{ Depression } \\
\hline No/Low & 345 (51.3) & $290(84.1)$ & 55 (15.9) & $256(74.2)$ & $89(25.8)$ \\
\hline Yes/High & $328(48.7)$ & $240(73.2)$ & $88(26.8)$ & $207(63.1)$ & $121(36.9)$ \\
\hline
\end{tabular}

In both the AACTG and VAS scales, the $t$-test with self-rated adherence found people that are adherent perceive their adherence to be better than people that are not adherent. When investigating the reasons for non-adherence, the logistic regression for the AACTG and VAS adherence indicator and the 14 possible reasons for missing medication (Chesney et al., 2000) shows only $12 \%$ variance meaning that the reasons are not predictors of adherence but merely excuses for not taking ARVs. The reason "Desire to avoid side effects," which was answered Yes by $13.5 \%$ $(N=91)$ of respondents, showed significance $(p=<0.05)$ for both AACTG and VAS, pointing out that people may avoid their ARVs due to side effects.

In Table 3, the bivariate logistic regression, the both the AACTG and the VAS adherence measure shows associations between age, alcohol usage of less than two alcoholic drinks on at least on one occasion in the previous four weeks, disclosure of HIV status to anyone, higher HIV knowledge, fear of side effects of the ARV medication, low levels of stigma, and depression. Additionally, the bivariate logistic regression with AACTG showed association with more than 12 months on ARVs as well as no IPV. All variables that were found to be statistically significant in the bivariate analysis were put into a multivariate model looking at associations for AACTG and VAS adherence (see Table 3). In the multivariate model, older age was associated with increased ARV adherence on both the AACTG and VAS measure, 
as well as drinking less than two alcoholic drinks on at least on one occasion in the previous four weeks. The multivariate model on both the AACTG and VAS measure goes on to show significant associations with disclosure of HIV status to anyone, higher HIV knowledge as well as no or low depression. Chances of non-adherence are shown with both the AACTG and VAS measure to increase due to a desire to avoid side effects of the ARV medication.

Table 3: Association of demographic, socioeconomic, health, and behaviour characteristics to ARV non-adherence.

\begin{tabular}{|c|c|c|c|c|}
\hline \multirow[t]{2}{*}{ Variable } & \multicolumn{2}{|c|}{ AACTG Non-Adherence } & \multicolumn{2}{|l|}{ VAS Non-Adherence } \\
\hline & $\operatorname{Cr}$ OR $(95 \% \mathrm{Cl}) * p$ & Adj OR $(95 \% \mathrm{Cl}) * p$ & $\operatorname{Cr}$ OR $(95 \% \mathrm{Cl}) * p$ & Adj OR $(95 \% \mathrm{Cl}) * p$ \\
\hline Age (scale) & $\begin{array}{l}1.06(1.02-1.10) \\
* p=0.001\end{array}$ & $\begin{array}{l}1.05(1.02-1.09) \\
* p=0.006\end{array}$ & $\begin{array}{l}1.04(1.01-1.08) \\
* p=0.004\end{array}$ & $\begin{array}{l}1.04(1.01-1.08) \\
* p=0.009\end{array}$ \\
\hline \multicolumn{5}{|l|}{ Language } \\
\hline $\begin{array}{l}\text { Nguni languages } \\
\text { Other languages }\end{array}$ & \multicolumn{3}{|c|}{ Other languages } & \\
\hline \multicolumn{5}{|l|}{ Educational attainment } \\
\hline $\begin{array}{l}\text { Grade } 12 \text { or more } \\
\text { Grade } 11 \text { and less }\end{array}$ & \multicolumn{3}{|c|}{ Grade 11 and less } & \\
\hline \multicolumn{5}{|l|}{ Relationship status } \\
\hline \multicolumn{4}{|l|}{ Living together } & \\
\hline \multicolumn{5}{|l|}{ Employment status } \\
\hline $\begin{array}{l}\text { Not employed } \\
\text { Employed, student, } \\
\text { volunteer }\end{array}$ & $1.05(0.67-1.64)$ & & $1.02(0.69-1.15)$ & \\
\hline \multicolumn{5}{|c|}{ Income (ZAR) per month } \\
\hline $\begin{array}{l}<\text { R310 } \\
\text { R311 or more }\end{array}$ & \multicolumn{3}{|c|}{ R311 or more } & \\
\hline \multicolumn{5}{|l|}{ Number of children } \\
\hline \multicolumn{5}{|l|}{ One or more } \\
\hline \multicolumn{5}{|l|}{ Unplanned pregnancy } \\
\hline \multicolumn{4}{|l|}{ Yes } & \\
\hline \multicolumn{5}{|c|}{ Alcohol use of $\mathbf{2}$ or more drinks on at least one occasion in the previous four weeks } \\
\hline $\begin{array}{l}\text { No } \\
\text { Yes }\end{array}$ & $\begin{array}{l}0.35(0.22-0.56) \\
* p=0.000\end{array}$ & $\begin{array}{l}0.43(0.26-0.71) \\
* p=0.001\end{array}$ & $\begin{array}{l}0.41(0.27-0.65) \\
* p=0.000\end{array}$ & $\begin{array}{l}0.49(0.31-0.79) \\
* p=0.003\end{array}$ \\
\hline \multicolumn{5}{|c|}{ Diagnosed with HIV in this pregnancy } \\
\hline $\begin{array}{l}\text { No } \\
\text { Yes }\end{array}$ & \multicolumn{4}{|c|}{ Yes } \\
\hline \multicolumn{5}{|c|}{ Time on ARV medication } \\
\hline $\begin{array}{l}<12 \text { months } \\
\geq 12 \text { months }\end{array}$ & $\begin{array}{l}1.73(1.08-2.77) \\
* p=0.023\end{array}$ & $\begin{array}{l}1.29(0.77-2.18) \\
p=0.340\end{array}$ & $1.31(0.89-1.94)$ & \\
\hline \multicolumn{5}{|c|}{ Desire to avoid side effects } \\
\hline No & $\begin{array}{l}0.36(0.23-0.57) \\
* p=0.000\end{array}$ & $\begin{array}{l}0.42(0.25-0.79) \\
* p=0.001\end{array}$ & $\begin{array}{l}0.36(0.23-0.57) \\
* p=0.000\end{array}$ & $\begin{array}{l}0.39(0.24-0.63) \\
* p=0.000\end{array}$ \\
\hline
\end{tabular}

Yes 
Table 3: Association of demographic, socioeconomic, health, and behaviour characteristics to ARV non-adherence (cont...)

\begin{tabular}{|c|c|c|c|c|}
\hline \multirow[t]{2}{*}{ Variable } & \multicolumn{2}{|c|}{ AACTG Non-Adherence } & \multicolumn{2}{|l|}{ VAS Non-Adherence } \\
\hline & $\operatorname{Cr}$ OR $(95 \% \mathrm{Cl}) * p$ & $\operatorname{Adj}$ OR $(95 \% \mathrm{Cl}) * p$ & $\operatorname{Cr}$ OR $(95 \% \mathrm{Cl}) * p$ & Adj OR $(95 \% \mathrm{Cl}) * p$ \\
\hline \multicolumn{5}{|c|}{ Disclosure of HIV serostatus to anyone } \\
\hline No & $\begin{array}{l}1.87(1.26-2.76) \\
* p=0.002\end{array}$ & $\begin{array}{l}1.76(1.16-2.68) \\
* p=0.008\end{array}$ & $\begin{array}{l}1.72(1.21-2.45) \\
* p=0.003\end{array}$ & $\begin{array}{l}1.72(1.18-2.50) \\
* p=0.004\end{array}$ \\
\hline \multicolumn{5}{|l|}{ Yes } \\
\hline \multicolumn{5}{|l|}{ HIV-positive children } \\
\hline No & $0.80(0.30-2.16)$ & & $1.47(0.67-3.20)$ & \\
\hline \multicolumn{5}{|l|}{ Yes } \\
\hline \multicolumn{5}{|l|}{ HIV-positive partner } \\
\hline No or Don't Know & $0.68(0.35-1.32)$ & & $0.74(0.42-1.31)$ & \\
\hline \multicolumn{5}{|l|}{ Yes } \\
\hline \multicolumn{5}{|l|}{ Male involvement } \\
\hline No & $1.17(0.81-1.70)$ & & $1.16(0.84-1.62)$ & \\
\hline \multicolumn{5}{|l|}{ Yes } \\
\hline \multicolumn{5}{|l|}{ HIV knowledge } \\
\hline No/Low & $1.76(1.21-2.55)$ & $1.46(0.98-2.17)$ & $1.72(1.24-2.40)$ & $1.47(1.04-2.09)$ \\
\hline INO/LOW & $* p=0.003$ & $p=0.060$ & $* p=0.001$ & $* p=0.030$ \\
\hline \multicolumn{5}{|l|}{ High } \\
\hline \multicolumn{5}{|c|}{ Intimate partner violence } \\
\hline No & $\begin{array}{l}0.60(0.39-0.92) \\
* p=0.019\end{array}$ & $\begin{array}{l}0.79(0.49-1.27) \\
p=0.337\end{array}$ & $1.45(0.98-2.16)$ & \\
\hline \multicolumn{5}{|l|}{ Yes } \\
\hline Stigma (Scale) & $\begin{array}{l}0.67(0.46-0.97) \\
* p=0.035\end{array}$ & $\begin{array}{l}0.79(0.56-1.25) \\
p=0.389\end{array}$ & $\begin{array}{l}0.69(0.50-0.97) \\
* p=0.030\end{array}$ & $\begin{array}{l}0.82(0.58-1.16) \\
p=0.254\end{array}$ \\
\hline \multicolumn{5}{|l|}{ Depression } \\
\hline & $0.52(0.35-0.76)$ & $0.63(0.42-0.948)$ & $0.60(0.43-0.83)$ & $0.71(0.50-1.00)$ \\
\hline No/Low & $* p=0.001$ & $* p=0.023$ & $* p=0.002$ & $* p=0.049$ \\
\hline Yes/High & & & & \\
\hline
\end{tabular}

* $95 \%$ significance.

\section{Discussion}

The high non-adherence (21\% AACTG non-adherence and 31\% VAS non-adherence) to ARV medication by HIV-positive pregnant women is of serious concern. There were a few factors that were found to contribute to non-adherence. The intake of alcohol has been shown to decrease ARV adherence (Kreitchmann et al., 2012; Mellins et al., 2008; Nachega et al., 2012); in this study, for those respondents that consumed less than two alcoholic drinks on at least on one occasion in the previous four weeks, adherence to ARVs was more likely than those that consumed more than two alcoholic drinks on one occasion. Alcohol is widely accepted as a disinhibitor, and this study shows that drinking less alcohol increases adherence to ARVs among pregnant women. It would seem that, as the pregnant women consume alcohol, they either forget about their ARVs, cannot take it due to the lack of privacy of their drinking location, or cannot take it due to the alcohol/ARV interaction. 
Non-adherence to ARVs was also more likely for those who wanted to avoid the side effects of the ARV medication. As mentioned earlier, early stages of pregnancy are characterized by morning sickness and late stages with heartburn (Nachega et al., 2012), and these conditions could be exacerbated by the side effects of ARVs, which include but are not limited to nausea, vomiting, and gastro-intestinal issues (Nachega et al., 2012). To ease discomfort, it is easy to assume that some pregnant women may not adhere to the ARV treatment regime.

Disclosure, or rather the lack of disclosure, of ones HIV-positive status to anyone was strongly associated with non-adherence of ARV medication. For those that do disclose, Futteman et al. (2010) and Nchenga et al. (2012) show that social support leads to decreased depression, which in turns leads to increased adherence to ARVs. Depression is a contributor to ARV adherence (Mellins et al., 2008; Nachega et al., 2012); in rural Mpumalanga, we found that those who reported lower depression had better adherence to ARVs. The PMTCT protocol does make provisions for emotional well-being, where it is seen as protective to the mother and ultimately the unborn child (Futterman et al., 2010). It stands to reason that a pregnant woman with little or no depressive symptoms will be more likely to be adherent to her ARVs than a depressed pregnant woman. It must also be noted that the majority (75\%) of the study respondents initiated Efavirenz-based ARV medication within the previous 12 months and that $54 \%$ were diagnosed HIV-positive during this pregnancy. These factors could contribute to depression. Interestingly, all of the above-mentioned contributing factors to ARV adherence could be related to the amount of HIV knowledge a respondent may or may not have. The study data clearly shows that non-adherence was related to no/low HIV knowledge. With knowledge, people are empowered to act to protect themselves and their unborn child (Futterman et al., 2010).

An important factor for increased adherence to ARV was that of older age. The results indicate that respondents who were of an older age were more likely to be adherent than younger respondents in our sample (Kreitchmann et al., 2012; Nachega et al., 2012) and seem to suggest increased adherence responsibility with age.

\section{Conclusions}

In order to achieve a PMTCT goal of first reducing and then eliminating HIV transmission from mother to child, thereby ensuring all babies born are HIVnegative and remain that year for their first year of their lives, adherence to ARV is a necessary step. It is of great concern to find a high percentage of the pregnant HIVpositive respondents in this study to be non-adherent to their ARV medication. Older respondents were found to be more likely to be adherent then younger respondents. Participants who reported increased alcohol intake, those who showed no to lower 
HIV knowledge, those who wanted to avoid the ARV side effects, those who did not disclosure their HIV status, and those who showed increased depression were more likely to be non-adherent.

Due to the findings, it is suggested that programs that encourage disclosure of one's HIV and pregnancy status are still needed and need to be strengthened. It is also advised that programs targeting prenatal alcohol intake as well as programs designed to help mothers with prenatal depression as well as HIV depression also need to be investigated and encouraged. It is also advised that more, closer monitoring is needed with regard to the ARV side effects to mitigate its effects and increase adherence. More ongoing education campaigns are definitely needed and will assist with disclosure, alcohol intake, depression, ARV side effects, and any other barriers to better adherence to ARV treatment. More research is needed on adherence to ARV among different populations in South Africa and the world, as there is currently limited research. 


\section{CHAPTER 3}

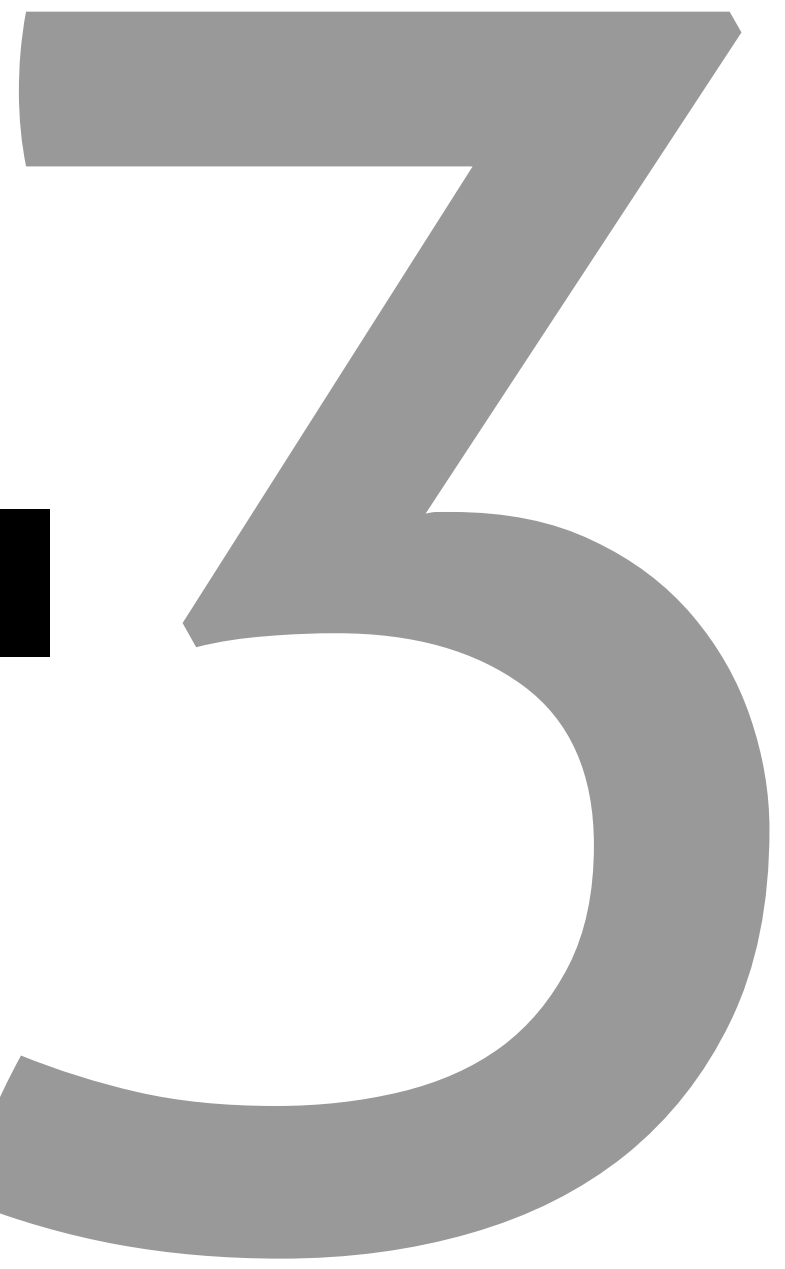




\section{DETERMINANTS OF DISCLOSURE AND NON- DISCLOSURE OF HIV-POSITIVE STATUS, BY PREGNANT WOMEN IN RURAL SOUTH AFRICA}

Published as:

Ramlagan, S., Matseke, G., Rodriguez, V. J., Jones, D. L., Peltzer, K., Ruiter, R.A.C., \& Sifunda, S. (2018). Determinants of disclosure and non-disclosure of HIV-positive status, by pregnant women in rural South Africa. 


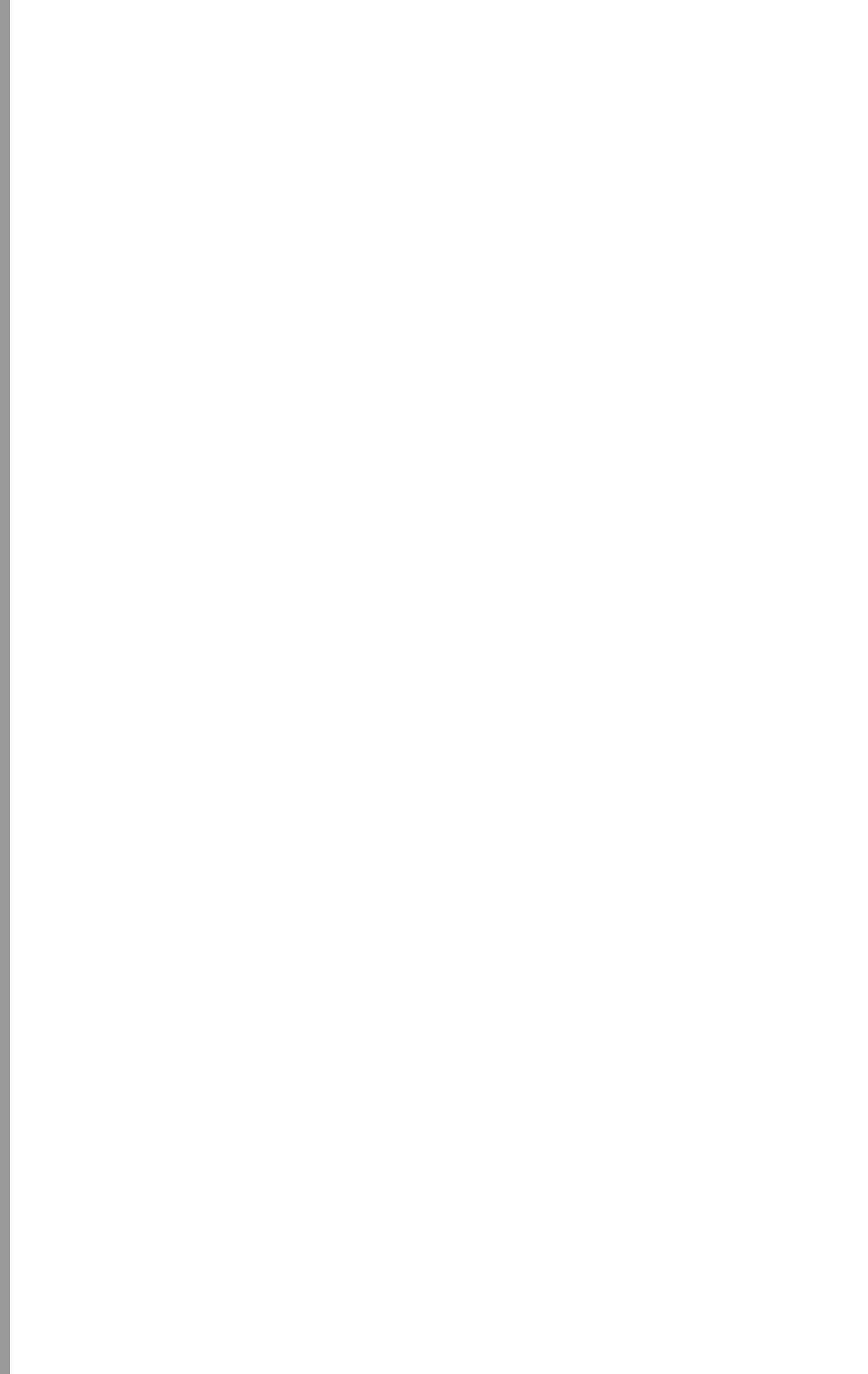




\section{Introduction}

Disclosure of HIV serostatus has been emphasized as a crucial goal in HIV testing and counselling as well as prevention of mother to child transmission of HIV [PMTCT] protocols (UNAIDS, 2011; WHO, 2012; Alemayehu et al., 2014; Medley, Garciamoreno, Mcgill, \& Maman, 2004). An individual may disclose to family members, co-workers or friends for social and emotional support or sexual partners for HIV prevention as well as support. It is crucial for pregnant women to disclose to sexual partners for them to adopt safer sexual behaviours, to prevent re-infection if they are both HIV infected, or avoid infecting the HIV negative partner (Shiyoleni, 2013). In addition, HIV disclosure to sexual partners minimize the risk of HIV transmission to the unborn baby during pregnancy, at delivery and during breastfeeding after birth (Shiyoleni, 2013). Disclosure by HIV positive women can encourage their partners to make informed reproductive health choices (Shiyoleni, 2013).

Mathematical models have shown that serostatus disclosure may contribute to the reduction of HIV transmission by $41 \%$ (Pinkerton \& Galletly, 2007). The percentage of women who disclose their status to at least one person varies widely across regions. Prevalence rates of HIV serostatus disclosure to any person ranged from 5.0\% to 96.7\% (Kiula, Damian, \& Msuya, 2013; Patel et al., 2012; Tam, Amzel, \& Phelps, 2015). Among pregnant women prevalence of HIV serostatus disclosure to sexual partners ranged from 16.7\% - 78\% (Brou et al., 2007; Desgrées-du-Loû et al., 2009; Gaillard et al., 2002; Kilewo et al., 2001; Kiula et al., 2013; Nebié et al., 2001). In a South African study among HIV positive new mothers in Nkangala district, $79 \%$ reported that they disclosed their status to someone and $65 \%$ disclosed to their partners (Peltzer \& Mlambo, 2013). A study conducted in Zimbabwe reported that $78 \%$ of women had disclosed their status to their current partner and the majority (85-98\%) experienced a positive reaction (Patel et al., 2012). In contrast, a study conducted in Sub-Saharan Africa reported much lower rates of disclosure to partner, at 37\%, among HIV infected pregnant women (Hardon et al., 2012).

Disclosure of their HIV status by women to their male partner was found to significantly improve antiretroviral adherence during pregnancy in Tanzania (Kirsten et al, 2011) and Nigeria (Ekama et al, 2012). There were also significant increases to adherence to the PMTCT protocol in the post-natal period, such as returning for post counseling, increased adherence to ART medication, and increased condom use (Ramirez-Ferrero, et al,. 2012; Patel et al., 2012), as well as lower levels of depression (Patel et al., 2012). It is possible that these reported improvements in adherence as a result of HIV serostatus disclosure may, in part, be due to increased male involvement and support. Increasing male partner involvement (MPI) in PMTCT programs can help women and their partners to understand and cope with the diagnosis (Albrecht et al., 2006; Maru et al., 2009; Sagay, Musa, Ekwempu, \& Imade, 2006). In South Africa, disclosure and male partner involvement have similarly been shown to improve antiretroviral adherence during pregnancy among 
HIV positive women (Peltzer et al., 2011).

Despite several benefits associated with HIV serostatus disclosure, there are various barriers that prevent women from disclosing to their sexual partners. In South Africa, significant progress has been achieved in the implementation of PMTCT programs. However, these improvements have, for the most part, occurred in urbanized areas, with rural areas remaining at unacceptably high levels of MTCT of HIV (Wettstein et al., 2012). Lack of HIV status disclosure has been identified as one of the major barriers to PMTCT effectiveness in rural areas (Tabana et al., 2012; Mepham et al., 2011; Bancheno et al., 2010; Bajunirwe et al., 2005; Jones et al., 2014; Rollins et al., 2007) as well as posing as a challenge to implementation of PMTCT programs in overburdened clinics (Kuonza et al., 2011; Turan et al., 2011; 2012; Hardon et al., 2012).

Studies among HIV infected women have shown that HIV disclosure could lead to undesirable outcomes such as intimate partner violence (Colombini, James, Ndwiga, \& Mayhew, 2016; Medley et al., 2004; Shamu, Zarowsky, Shefer, Temmerman, \& Abrahams, 2014), stigma and discrimination(French, Greeff, \& Watson, 2015). Being diagnosed HIV positive has also been shown to cause post-traumatic stress (Olley, Zeier, Seedat, \& Stein, 2005). A review of 17 studies (15 in Sub Saharan Africa and 2 from South East Asia) showed that between 3.5\% and 14.6\% of women reported experiencing a violent reaction from a partner following disclosure (Medley et al, 2004).

A review of factors associated with HIV serostatus disclosure in Sub-Saharan Africa (Tam et al., 2015) identified four main determinants, i.e., factors related to the woman herself (younger age, first pregnancies, knowing someone with HIV, lower levels of internalized stigma, and lower levels of avoidant coping), the partner (prior history of HIV testing and higher levels of educational attainment), their partnership (no history of domestic violence and financial independence), and the household (higher quality of housing and residing without spouses or extended family members). On the contrary, other studies have shown low rates of disclosure among younger women compared to older women (Kadowa \& Nuwaha, 2009; Ssali et al., 2010).

In a South African study among HIV positive pregnant women, being married, prior discussion about testing, having a partner with tertiary education and less experience of violence were identified as factors associated with having disclosed to partners (Makin et al, 2008). Peltzer and Mlambo (2013) found that among HIV positive new mothers in South Africa, having been diagnosed HIV positive more than two years ago and knowing their partner is HIV positive among other factors, increased the likelihood of disclosure. Better housing, low financial dependence on partners, and knowing someone with HIV were associated with disclosure to any 
other person (Makin et al, 2013). In Tanzania, HIV-disclosure to partners was more likely among pregnant women who were under 25 years old, who knew their HIV status before the current pregnancy, and discussed with their partner before testing (Kiula et al., 2013). Dependency on the partner for food/rent/school fees, led to lower odds of disclosure to partners (Kiula et al., 2013).

This study examines the socio-demographic, HIV related, and psychosocial determinants of HIV status disclosure and non-disclosure among HIV sero-positive pregnant women in rural South Africa.

\section{Methodology}

\section{Design}

The methodology and data presented here forms part of the Protect Your Family (PYF) randomized controlled trial described in detail elsewhere (Jones et al., 2014). The study was conducted in Mpumalanga province, South Africa. Within the province, 12 community health centres ( $\mathrm{CHC}$ ) from the Nkangala District Municipality and Gert Sibande District Municipality were selected as study sites. As per the study protocol, "twelve CHCs were matched in a 1:1 ratio according to patient census and average ANC volume, and one clinic in each pair was randomly assigned to the experimental or control condition using a computer program written by the data manager. The matched clinics were then assigned to the opposite condition" (Jones et al., 2014, p.415). Over a 12 month period, from April 2014 to March 2015, crosssectional data were gathered.

\section{Sample and procedure}

A total of 673 women were recruited by trained PYF staff and enrolled into this study. In order to be enrolled, women had to be aged 18 years or older, less than six months pregnant, HIV-seropositive, and having a current partner. Gestational age was determined by $\mathrm{CHC}$ nurses utilizing anti-natal protocols and HIV seropositive status was determined utilizing South African Department of Health PMTCT guidelines (Department of Health, 2014). It was not necessary for the current partner to be the father of the unborn baby. Once a potential respondent was identified, they were introduced to the study information sheet by the trained PYF staff member, in the language of their preference including English, isizulu, or seSotho. The study information sheet was read out to them while they followed on their own copy. Respondents were given an opportunity to ask questions after each paragraph. The information sheet and consent form ensured participants knew that confidentiality was of the highest priority in this study and their HIV status and data they provided could not be linked to them personally. Upon agreeing to participate, they were asked to provide written informed consent. 
Briefly, enrolled participants then completed the assessment in their above mentioned preferred language utilising the Questionnaire Development System v3.0 (QDS ${ }^{\mathrm{TM}}$ ) Audio Computer-Assisted Self-Interview (ACASI) software (NIMH Multisite HIV/STD Prevention Trial for African American Couples Group, 2008). All participants were provided with over-ear headphones and given brief training by the PYF staff member on the usage of the laptop touchscreen, headphones, and ACASI software. Once the assessment was completed, the PYF staff member saved the data onto the touch screen laptop utilizing a unique study participant identification number as well as date when the interview occurred. Participants were then thanked for their time and compensated with R50.00 (South African Rand= US\$4.72). The data were backed up onto an external memory drive at the end of every day by the PYF staff member at each CHC. Once every week, the data were collected in person from each CHC by a PYF study coordinator and transferred to the study head office in Pretoria and handed over to the study statistician.

\section{Ethical Approval}

Ethical approval was granted by the Human Sciences Research Council (HSRC) Research Ethics Committee (REC), protocol approval number REC4/21/08/13 as well as the University of Miami, Miller School of Medicine, Institutional Review Board (IRB ID: 20130238 (CR00006122). As the entire study was being conducted within Mpumalanga Province, South Africa, study approval was also obtained from the Department of Health and Welfare, Mpumalanga Provincial Government, South Africa.

\section{Measures}

\section{Primary outcome measure}

Disclosure of HIV status which is the primary outcome was derived from two independent questions regarding the disclosure of the participants HIV positive sero-status. The first outcome question was "have you disclosed your HIV status to anyone (someone)," with response options Yes or No. This question also included disclosure to one partner. The second outcome question was "have you disclosed your HIV status to your partner," with response options Yes vs No. This study defined a male partner as either the women's husband, or the current baby's father, or the women's current male sexual partner, or the women's trusted male friend who was actively involved in her life (Jones et al., 2014).

\section{Explanatory measures}

Socio-demographic items were assessed with questions including age, language (Nguni vs others), education (completed Grade 11 or less and completed Grade 12 or more), relationship status (living together with current partner or not), employment status (not employed versus employed, volunteer or student), income per month in South African Rand (ZAR) where the South African Government child grant was R310.00 (UD\$29.27) during the study period; number of children (none 
vs one or more); planned or unplanned pregnancy; and alcohol use of more than 2 drinks on at least on one occasion in the past 4 weeks (yes vs no).

HIV related measures included being diagnosed with HIV during this pregnancy (yes vs no); if you have a living child, are any of your children HIV positive (yes vs no); and is your current partner HIV positive (yes vs no). An adapted version of the Visual Analog Scale (VAS; Giordano et al., 2004) was utilized to measure adherence to antiretroviral therapy (ART). Adherence was noted as $100 \%$ if all ART doses were taken over the previous seven days. Range was from $0 \%$ (not taken on any single day) to $100 \%$ (7 days). Stigma was assessed using the nine-item AIDS-Related Stigma Scale (ARSS; Kalichman et al., 2005). Scores on the scale range from 0 to 8 , where higher scores indicate greater levels of stigma. Cronbach's Alpha reliability coefficient for the scale in this study was $\alpha=.73$.

Intimate partner violence (IPV) was assessed using an adapted 18-item version of the Conflict Tactics Scale 18 (CTS-18; Strauss et al., 1979). The scale asked to rate the number of times she and her partner may have engaged in the conflict in the past month on a seven point scale of 0 (Never) to 6 (More than 20 times). A higher score, is indicative of increased IPV. Cronbach's Alpha reliability coefficient for the scale in this study was $\alpha=.84$. Mental status was assessed using the 10-item Edinburgh Postnatal Depression Scale 10 (EPDS-10; Cox et al., 1987). Scores range from 0 to 30, where the higher the score, the more the likelihood of depression being experienced. This paper utilised the validated cut-off score for South African populations at score 12 (Lawrie et al., 1998). Cronbach's Alpha reliability coefficient for the scale in this study was $\alpha=.66$. Male involvement was assessed using a Yes/No item to enquire whether or not the respondent's male partner knew what occurred during in the antenatal clinic visit.

\section{Data analysis}

Summary statistics (means, frequencies and percentages) were used to describe the study sample utilizing SPSS version 24.0 for Windows (I.B.M Corp, 2016). Cronbach's Alpha reliability coefficients were calculated for scales used in the analyses, including IPV, ARSS, and EPDS scales. Bivariate analyses and multivariate logistic regressions were used to investigate associations between the primary outcome, disclosure of HIV status, and socioeconomic, HIV related, and behavioural variables. Associations were considered significant at $P<.05$. All statistically significant variables in the bivariate analyses were included in the multivariate model.

\section{Results}

\section{Sample characteristics and HIV disclosure}

The 673 HIV positive pregnant women in this study had a mean age of 28.39 years $(S D=5.73)$, with the majority (58\%) between the ages of $18-29$ years old. Over 
two-thirds (72.1\%) disclosed their status to someone, while just over half $(58.4 \%)$ disclosed to their partners. All respondents indicated they were in a relationship with a partner, however a high proportion of about $62 \%$ were not living together with their partner. Among those respondents who were not living together with their partner, $47 \%$ did not disclose their HIV positive status to their partner and among those living with their partners, 34\% did not disclose their HIV positive status to their partner they are living with. About $78 \%$ reported that they were unemployed and a third reported earning a monthly income of less than R310. Of the respondents that earned less than R310 per month, 33\% did not disclose their HIV positive status to someone and $44 \%$ did not disclose their status to their partners (see table 1 ). A fifth (21\%) of respondents reported that they had no children and of these, $63 \%$ disclosed their HIV status to someone and half $(50 \%)$ disclosed to their partner.

Table 1: Sample characteristics and HIV disclosure

\begin{tabular}{|c|c|c|c|c|c|}
\hline \multirow[b]{3}{*}{ Sociodemographics } & \multirow{3}{*}{$\begin{array}{l}\text { Sample } \\
\mathrm{N}(\%)\end{array}$} & \multicolumn{2}{|c|}{$\begin{array}{l}\text { Disclosed HIV status to } \\
\text { Someone }\end{array}$} & \multicolumn{2}{|c|}{$\begin{array}{c}\text { Disclosed HIV status to } \\
\text { Partner }\end{array}$} \\
\hline & & Yes & No & Yes & No \\
\hline & & $n(\%)$ & $n(\%)$ & $n(\%)$ & $n(\%)$ \\
\hline All & $673(100)$ & $485(72.1)$ & $188(27.9)$ & $393(58.4)$ & $280(41.6)$ \\
\hline \multicolumn{6}{|l|}{ Language } \\
\hline Nguni languages & $546(81.1)$ & $397(72.7)$ & $149(27.3)$ & $318(58.2)$ & $228(41.8)$ \\
\hline Other languages & $127(18.9)$ & $88(69.3)$ & $39(30.7)$ & $75(59.1)$ & $52(40.9)$ \\
\hline \multicolumn{6}{|l|}{ Educational attainment } \\
\hline Grade 12 and more & $192(28.5)$ & $141(73.4)$ & $51(26.6)$ & $117(60.9)$ & $75(39.1)$ \\
\hline Grade 11 and less & $481(71.5)$ & $344(71.5)$ & $137(28.5)$ & $276(57.4)$ & $205(42.6)$ \\
\hline \multicolumn{6}{|l|}{ Relationship status } \\
\hline Not living together & $419(62.3)$ & 299(71.4) & $120(28.6)$ & $224(53.5)$ & $195(46.5)$ \\
\hline Living together & $254(37.7)$ & $186(73.2)$ & $68(26.8)$ & $169(66.5)$ & $85(33.5)$ \\
\hline \multicolumn{6}{|l|}{ Employment status } \\
\hline Not employed & $527(78.3)$ & $379(71.9)$ & $148(28.1)$ & $314(59.6)$ & $213(40.4)$ \\
\hline Employed, Volunteer or Student & $146(21.7)$ & $106(72.6)$ & $40(27.4)$ & $79(54.1)$ & $67(45.9)$ \\
\hline \multicolumn{6}{|l|}{ Income (ZAR)per month } \\
\hline <R310 (child grant) & $221(32.8)$ & $148(67.0)$ & $73(33.0)$ & $123(55.7)$ & $98(44.3)$ \\
\hline R311 or more & $452(67.2)$ & $337(74.6)$ & $115(25.4)$ & $270(59.7)$ & $182(40.3)$ \\
\hline \multicolumn{6}{|l|}{ Number of living children } \\
\hline None & $139(20.7)$ & $88(63.3)$ & $51(36.7)$ & $70(50.4)$ & $69(49.6)$ \\
\hline One or more & $534(79.3)$ & $397(74.3)$ & $137(25.7)$ & $323(60.5)$ & $211(39.5)$ \\
\hline \multicolumn{6}{|l|}{ Unplanned pregnancy } \\
\hline No & $317(47.1)$ & $226(71.3)$ & $91(28.7)$ & $184(58.0)$ & $133(42.0)$ \\
\hline Yes & $356(52.9)$ & $259(72.8)$ & $97(27.2)$ & $209(58.7)$ & $147(41.3)$ \\
\hline
\end{tabular}


Table 1: Sample characteristics and HIV disclosure (cont...)

\begin{tabular}{lllllll} 
& Sample & \multicolumn{2}{c}{$\begin{array}{c}\text { Disclosed HIV status to } \\
\text { Someone }\end{array}$} & \multicolumn{2}{c}{$\begin{array}{c}\text { Disclosed HIV status to } \\
\text { Partner }\end{array}$} \\
& $\mathrm{N}(\%)$ & $\mathrm{n}(\%)$ & $\mathrm{n}(\%)$ & $\mathrm{n}(\%)$ & $\mathrm{n}(\%)$ \\
\hline Sociodemographics & $581(86.3)$ & $419(72.1)$ & $162(27.9)$ & $341(58.7)$ & $240(41.3)$ \\
\hline Alcohol use of more than $\mathbf{2}$ drinks on at least on one occasion in the past 4 weeks \\
No \\
Yes & $92(13.7)$ & $66(71.7)$ & $26(28.3)$ & $52(56.5)$ & $40(43.5)$ \\
\hline
\end{tabular}

Health and behavior characteristics and HIV disclosure

In the study sample, just over half (54\%) of the respondents were diagnosed with HIV during the current pregnancy (see Table 2 below). Of these respondents, almost two thirds (63\%) disclosed their HIV status to someone yet only about half (49\%) disclosed their HIV status to their partner. Table 2 shows that well over two thirds (69\%) of HIV positive pregnant women were $100 \%$ adherent to their ART medication and of these adherent respondents, a high three quarters (76\%) disclosed their status to any one and just over three fifths (62\%) disclosed their HIV positive status to their partners. A quarter (25\%) of respondents reported that their partner was diagnosed as HIV positive and of these, a very high $87 \%$ disclosed their HIV status to their partner (see Table 2). Only 58\% of respondents stated that their partner is involved in their pregnancy as their partner knows what takes place during the ante-natal care clinic, and of these respondents, $68 \%$ declared their HIV positive status to their partner.

Table 2: Health and behaviour characteristics and HIV disclosure

\begin{tabular}{|c|c|c|c|c|c|}
\hline & \multirow[t]{2}{*}{ Sample } & \multicolumn{2}{|c|}{$\begin{array}{l}\text { Disclosed HIV status to } \\
\text { Someone }\end{array}$} & \multicolumn{2}{|c|}{$\begin{array}{c}\text { Disclosed HIV status to } \\
\text { Partner }\end{array}$} \\
\hline & & Yes & No & Yes & No \\
\hline & $N(\%)$ & $\mathrm{n}(\%)$ & $n(\%)$ & $\mathrm{n}(\%)$ & $\mathrm{n}(\%)$ \\
\hline All & $673(100)$ & $485(72.1)$ & $188(27.9)$ & $393(58.4)$ & $280(41.6)$ \\
\hline \multicolumn{6}{|c|}{ Diagnosed with HIV in this pregnancy } \\
\hline No, before & $308(45.8)$ & $255(82.8)$ & $53(17.2)$ & $214(69.5)$ & $94(30.5)$ \\
\hline Yes & $365(54.2)$ & $230(63.0)$ & $135(37.0)$ & 179(49.0) & $186(51.0)$ \\
\hline \multicolumn{6}{|c|}{ Has HIV positive children } \\
\hline No or do not know & $506(94.8)$ & $134(26.5)$ & $372(73.5)$ & $303(59.9)$ & $203(40.1)$ \\
\hline Yes & $28(5.2)$ & $3(10.7)$ & $25(89.3)$ & $20(71.4)$ & $8(28.6)$ \\
\hline \multicolumn{6}{|l|}{ *VAS adherence } \\
\hline non adherent & $210(31.2)$ & $135(64.3)$ & $75(35.7)$ & $104(49.5)$ & $106(50.5)$ \\
\hline $100 \%$ adherent & $463(68.8)$ & $350(75.6)$ & $113(24.4)$ & $289(62.4)$ & $174(37.6)$ \\
\hline \multicolumn{6}{|l|}{ HIV positive partner } \\
\hline No or do not know & $506(75.2)$ & $336(66.4)$ & $170(33.6)$ & $248(49.0)$ & $258(51.0)$ \\
\hline Yes & $167(24.8)$ & $149(89.2)$ & $18(10.8)$ & $145(86.8)$ & $22(13.2)$ \\
\hline
\end{tabular}


Table 2: Health and behaviour characteristics and HIV disclosure (cont...)

\begin{tabular}{|c|c|c|c|c|c|}
\hline & \multirow[t]{2}{*}{ Sample } & \multicolumn{2}{|c|}{$\begin{array}{l}\text { Disclosed HIV status to } \\
\text { Someone }\end{array}$} & \multicolumn{2}{|c|}{$\begin{array}{l}\text { Disclosed HIV status to } \\
\text { Partner }\end{array}$} \\
\hline & & Yes & No & Yes & No \\
\hline & $\mathrm{N}(\%)$ & $n(\%)$ & $\mathrm{n}(\%)$ & $\mathrm{n}(\%)$ & $n(\%)$ \\
\hline \multicolumn{6}{|l|}{ Intimate partner violence } \\
\hline $\begin{array}{l}\text { No mild or No severe physical } \\
\text { violence }\end{array}$ & $541(80.4)$ & $148(27.4)$ & $393(72.6)$ & $324(59.9)$ & $217(40.1)$ \\
\hline Mild or severe physical violence & 132(19.6) & $40(30.3)$ & $92(69.7)$ & $69(52.3)$ & $63(47.7)$ \\
\hline \multicolumn{6}{|l|}{ Stigma } \\
\hline No (Score $=0$ ) & $400(59.4)$ & $114(28.5)$ & $286(71.5)$ & $231(57.8)$ & $169(42.3)$ \\
\hline Yes (Score=1-8) & $273(40.6)$ & $74(27.1)$ & 199(72.9) & $162(59.3)$ & $111(40.7)$ \\
\hline \multicolumn{6}{|l|}{ Depression } \\
\hline EDS score of 0-12 & $345(51.3)$ & $257(74.5)$ & $88(25.5)$ & $213(61.7)$ & $132(38.3)$ \\
\hline EDS score of 13 and more & $328(48.7)$ & $228(69.5)$ & $100(30.5)$ & $180(54.9)$ & $148(45.1)$ \\
\hline \multicolumn{6}{|l|}{ Male involvement } \\
\hline No & $281(41.8)$ & $181(64.4)$ & $100(35.6)$ & $125(44.5)$ & $156(55.5)$ \\
\hline Yes & $392(58.2)$ & $304(77.6)$ & $88(22.4)$ & $268(68.4)$ & $124(31.6)$ \\
\hline
\end{tabular}

*VAS=Visual Analogue Scale

Association of demographic, socioeconomic, health and behaviour characteristics to disclosure of HIV status

In Table 3, the results from the univariate logistic regression indicated that older age, increased ART adherence, having an HIV positive partner, and male partner involvement, were positively associated with HIV status disclosure to someone and to partners. Additionally, having a child/children, and being diagnosed with HIV during the current pregnancy, were negatively associated with HIV status disclosure to someone and to partners. Furthermore, Table 3 goes on to show that income and relationship status were associated with HIV status disclosure to someone and HIV status disclosure to partner, respectively. Participants with an income of R311 and more per month were less likely to disclose their HIV-positive status to someone and those respondents who are living together with their partner were more likely to disclose their HIV-positive status to their partners.

Finally, in multivariate analysis, increase in ART adherence, HIV positive partner, and male involvement, were significantly associated with both disclosure of HIV status to someone and disclosure of HIV status to partner. Participants who were $100 \%$ adherent to ART, those who had an HIV positive partner, and those whose male partner was involved in current pregnancy were more likely to disclose their HIV status to someone and to the partner (see Table 3, column Adj OR). Multivariate analysis further showed that participants who were diagnosed HIV positive during current pregnancy were less likely to disclose their HIV status to someone nor to their partner. 
Table 3: Association of demographic, socioeconomic, health and behaviour characteristics to disclosure of HIV status

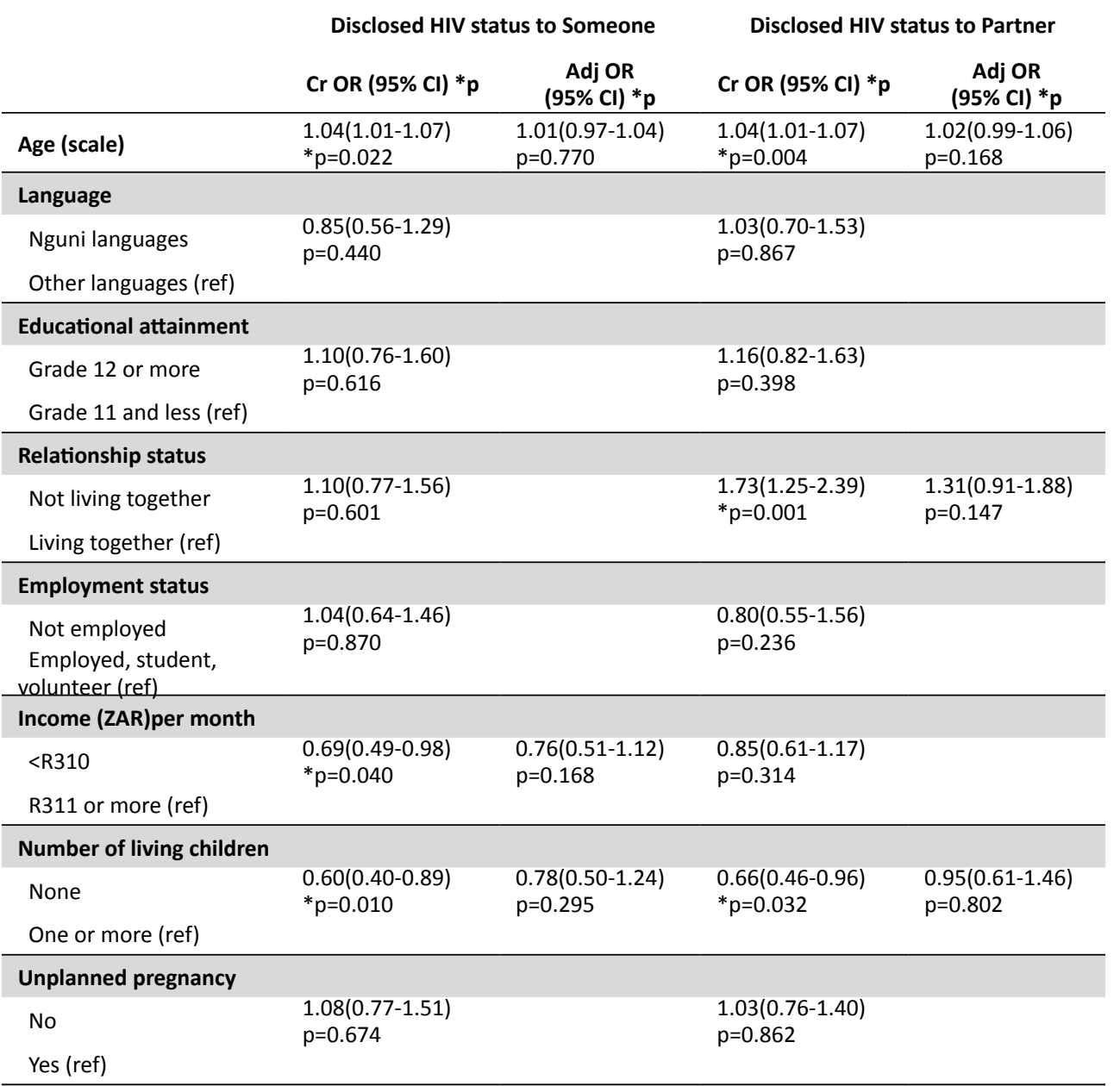

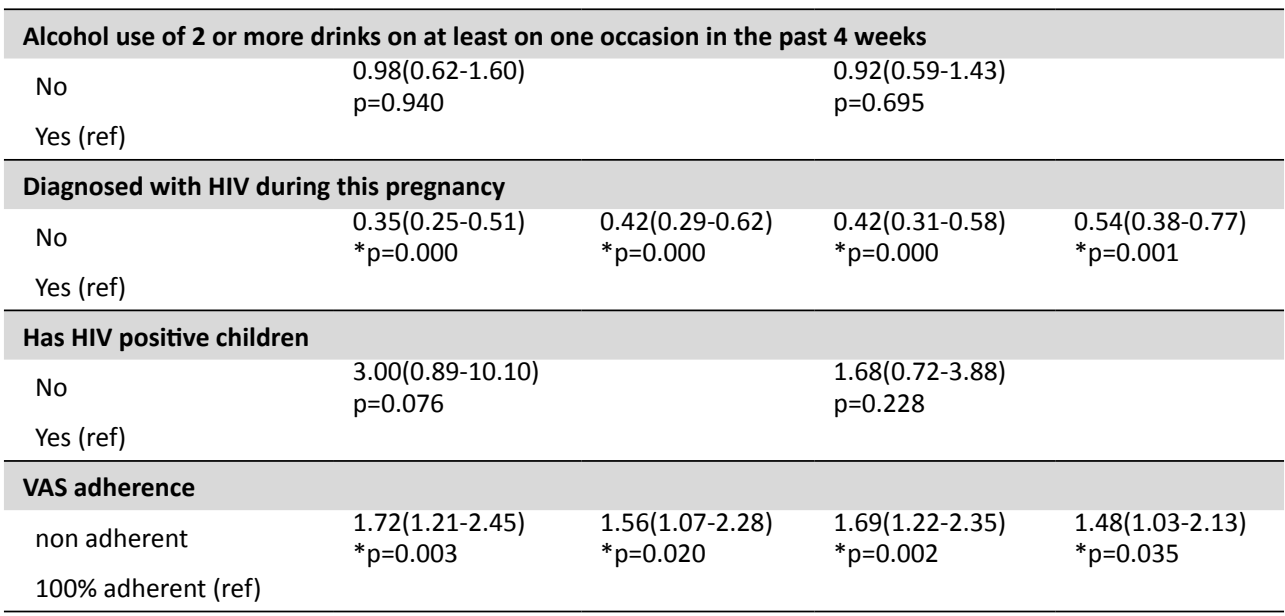


Table 3: Association of demographic, socioeconomic, health and behaviour characteristics to disclosure of HIV status (cont...)

\begin{tabular}{|c|c|c|c|c|}
\hline & \multicolumn{2}{|c|}{ Disclosed HIV status to Someone } & \multicolumn{2}{|c|}{ Disclosed HIV status to Partner } \\
\hline & $\operatorname{Cr}$ OR $(95 \% \mathrm{Cl}){ }^{*} \mathrm{p}$ & $\begin{array}{c}\text { Adj OR } \\
(95 \% \mathrm{Cl}) * p\end{array}$ & $\operatorname{Cr}$ OR $(95 \% \mathrm{Cl}){ }^{*} p$ & $\begin{array}{c}\text { Adj OR } \\
(95 \% \mathrm{Cl}) * p\end{array}$ \\
\hline \multicolumn{5}{|l|}{ HIV positive partner } \\
\hline $\begin{array}{l}\text { No or Don't Know } \\
\text { Yes (ref) }\end{array}$ & $\begin{array}{l}4.19(2.48-7.06) \\
* p=0.000\end{array}$ & $\begin{array}{l}2.94(1.71-5.06) \\
* p=0.000\end{array}$ & $\begin{array}{l}6.86(4.24-11.09) \\
{ }^{*} p=0.000\end{array}$ & $\begin{array}{l}4.99(3.03-8.21) \\
{ }^{*} p=0.000\end{array}$ \\
\hline \multicolumn{5}{|c|}{ Intimate partner violence } \\
\hline $\begin{array}{l}\text { No } \\
\text { Yes (ref) }\end{array}$ & $\begin{array}{l}0.87(0.57-1.31) \\
p=0.499\end{array}$ & & $\begin{array}{l}0.73(0.50-1.06) \\
p=0.112\end{array}$ & \\
\hline Stigma (Scale) & $\begin{array}{l}1.06(0.93-1.21) \\
p=0.393\end{array}$ & & $\begin{array}{l}1.07(0.95-1.20) \\
p=0.282\end{array}$ & \\
\hline \multicolumn{5}{|l|}{ Depression } \\
\hline $\begin{array}{l}\text { No/Low } \\
\text { High (ref) }\end{array}$ & $\begin{array}{l}0.78(0.56-1.09) \\
p=0.150\end{array}$ & & $\begin{array}{l}0.75(0.55-1.03) \\
p=0.071\end{array}$ & \\
\hline \multicolumn{5}{|l|}{ Male involvement } \\
\hline $\begin{array}{l}\text { No } \\
\text { Yes }\end{array}$ & $\begin{array}{l}1.91(1.36-2.68) \\
*_{p}=0.000\end{array}$ & $\begin{array}{l}1.67(1.17-2.40) \\
* p=0.005\end{array}$ & $\begin{array}{l}2.70(1.96-3.70) \\
* p=0.000\end{array}$ & $\begin{array}{l}2.34(1.66-3.30) \\
{ }^{*} p=0.000\end{array}$ \\
\hline
\end{tabular}

Cr OR= crude Odds Ratio; Adj OR = Adjusted Odds Ratio; *95\% significance

\section{Discussion}

This study examined the prevalence of HIV-positive status disclosure and nondisclosure among HIV positive pregnant women in rural South Africa. The results of this study highlight the high levels of overall non-disclosure, where just over two fifths of respondents did not disclose their HIV positive status to their male partners and over a quarter did not disclose to someone. A statistically significant association was found between those who were diagnosed with HIV during this current pregnancy, and both non-disclosure of their HIV-positive status to someone and non-disclosure of their HIV-positive status to their partner.

In line with PMTCT guidelines, women are initiated on ART on the same day as receiving an HIV diagnosis (Department of Health, 2014). Non-disclosure of HIV positive status among those newly diagnosed pregnant women has been attributed to post-traumatic stress disorder following HIV diagnosis (Olley et al., 2005). This occurs among many women testing HIV positive for the first time in pregnancy, during the antenatal period, despite counselling efforts. In this study, about half of the respondents were found to be depressed. This could be extremely traumatic as the respondents would have found out that they are pregnant and HIV positive almost around the same time they were being enrolled into the study. Most common reasons cited for non-disclosure of HIV status were not being emotionally 
ready to disclose, fear of negative reactions such as rejection, discrimination and violence (Visser et al., 2008). Non-disclosure places these pregnant women and their unborn child at greater risk of HIV reinfection, increased viral load, increased levels of depression, and decreases their potential support system emanating from the stigma and discrimination experiences (Shiyoleni, 2013).

This study found that over two thirds of the HIV positive pregnant women were $100 \%$ adherent to their ART medication. A strong positive relationship was also found between adherence to ART and disclosure of HIV status to someone and disclosure to partner. This finding is consistent with previous research in Mpumalanga province, South Africa (Peltzer et al., 2011), in Tanzania (Kirsten et al., 2011), and in Nigeria (Ekama et al., 2012). Increased MPI has been shown to help provide support for women with HIV diagnosis, which in turn, increase adherence to ART (Albrecht et al., 2006; Maru et al., 2009; Peltzer et al., 2011).

In fact, this study found a strong positive relationship between male partner involvement during pregnancy and disclosure of HIV status. Evidence shows that increasing positive male partner involvement in pregnancy can help women and their partners to understand and cope with the HIV diagnosis (Albrecht et al., 2006; Maru et al., 2009; Sagay et al., 2006). In addition, women who had an HIV positive partner were more likely to disclose their HIV status to their partners as well as to others. This finding implies that the partner could have also disclosed his HIV positive status at some point. Increased ability of women to safely disclose their HIV-positive status to male partners is therefore essential for increased uptake and effectiveness of PMTCT services (Tabana et al., 2012; Mepham et al., 2011; Bancheno et al., 2010).

\section{Limitations of the study}

A cross-sectional study design was used which is limited to determining the association between an outcome and risk factors, and therefore cannot infer causality between HIV status disclosure and associated factors. In addition, only self-report measures were used in the present study, which predisposes participants to social desirability bias. Nevertheless, the findings of this study adds to existing literature.

\section{Conclusion}

The findings of this study highlight the need to encourage disclosure as a way to promote ART adherence among HIV positive pregnant women. The findings also highlight the importance of male partner involvement during pregnancy in order to encourage disclosure. In addition, more research is needed on other ways to encourage disclosure among newly diagnosed HIV positive pregnant women during 
initiation in the PMTCT programme. This study recommends increased psychosocial support for newly diagnosed HIV positive pregnant women in order to encourage disclosure to their male partners. 

CHAPTER 4 


\section{CORRELATES OF PERCEIVED HIV-RELATED STIGMA AMONG HIV-POSITIVE PREGNANT WOMEN IN RURAL MPUMALANGA PROVINCE, SOUTH AFRICA}

Published as:

Ramlagan, S., Sifunda, S., Peltzer, K., Jean, J., \& Ruiter, R.A.C. (2019). Correlates of perceived HIV-related stigma among HIV-positive pregnant women in rural Mpumalanga province, South Africa. 


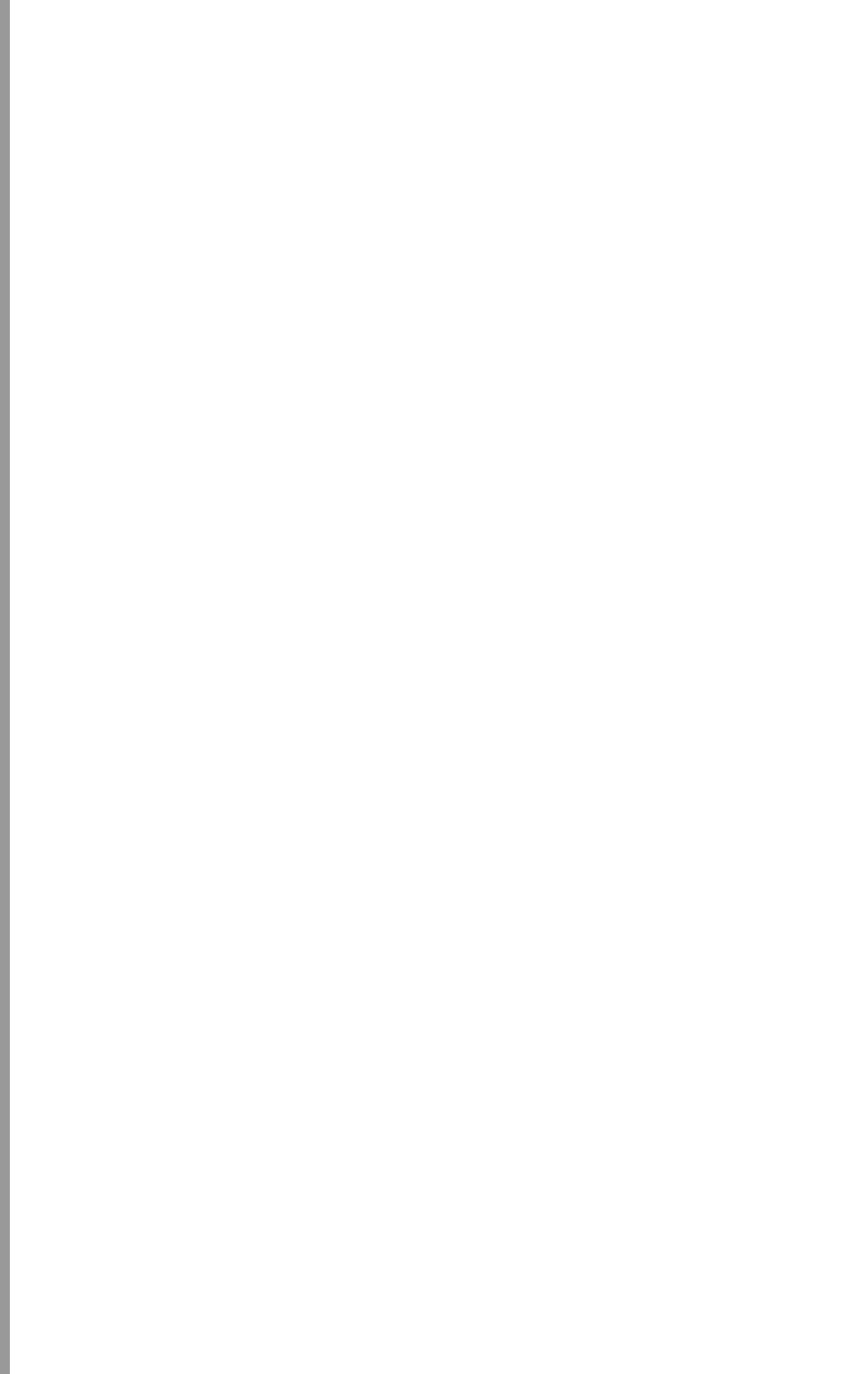




\section{Introduction}

Stigma has been defined as the possession of a devalued trait, behaviour or identity that results in the reduced status of individuals that possesses that trait, behaviour, or identity (Bos, Pryor, Reeder, \& Stutterheim, 2013; Brown, Macintyre, \& Trujillo, 2003; Hasan et al., 2012; Tsai, Bangsberg, \& Weiser, 2013). It is understood as an interactive process that occurs within the contexts of the environment, healthcare system and agent of said stigma and that both creates and reinforces pre-existing societal power structures (Holzemer et al., 2007). Stigma reside in the social context and is not considered to reside in a person (Bos et al., 2013). Stigma related to HIV is conceived as a product of the hegemonic discourses around 1) acquisition of HIV and personal accountability around said acquisition, 2) the lethality and spread of the virus, 3) visibility of being in a diseased state, and 4) fear of being associated with the disease or diseased (Alonzo \& Reynolds, 1995; Lee, Kochman, \& Sikkema, 2002; Novick, 1997). As such, it has "enormous negative side effects on social relationships, access to resources, social support provision, and psychological wellbeing" (Bos et al., 2008: 450). HIV-related stigma is also constructed and experienced at the intersection of other stigmas such as intravenous venous drug users, homosexuality, and sexual promiscuity/infidelity (Brown et al., 2003; Crawford, 1996; Novick, 1997).

Stigma is often thought to operate at three levels: enacted, anticipated, and internalized. Enacted stigma refers individuals' experience of current or past stereotyping, prejudiced and stigmatizing behaviours such as discrimination or exclusion due to their possession of the devalued trait (Bos et al., 2013; Earnshaw, Smith, Chaudoir, Amico, \& Copenhaver, 2013; Mbonye et al., 2013). Anticipated stigma is the expectation of future experiences of prejudice, stigmatizing behaviours, and stereotype due to one's possession of the devalued attribute (Earnshaw et al., 2013). Internalized stigma is defined as individuals' acceptance of the social construction of their attributes, behaviour or identity as undesirable and the ensuing reduction in status as legitimate (Earnshaw et al., 2013; Rao et al., 2012). Inherent to internalized stigma is the development of self-deprecating constructions of oneself through the embodiment of shame, guilt, blame and hopelessness (Brown et al., 2003; Earnshaw et al., 2013; Rao et al., 2012). Internalized HIV-related stigma is HIV positive individuals' acceptance and subsequent identification of their selves with the negative or devalued construction of what it means to be an HIV positive person (Kingori et al., 2012; Rao et al., 2012).

Internalised stigma needs to be measured with a valid and reliable scale. According to Lindberg, Wettergren, \& Wiklander (2014), the HIV stigma scale by Berger, Ferrans, \& Lashley (2001), differentiates between stigma dimensions in one instrument. The 40-item internalized stigma scale as tested and validated by Berger et al. (2001) in America and more recently by Lindberg et al. (2014) in Sweden produced four 
factors representing four dimensions of stigma. The factor analysis sequence of the four dimensions were (1) personalised stigma, (2) disclosure concerns, (3) negative self-image, and (4) concerns with public attitudes (Berger et al., 2001; Lindberg et al., 2014). Both studies had a different number of item loadings for each dimension with Berger et al. (2001) and Lindberg et al. (2014) reporting personalised stigma with 18 -items vs 16 -items, disclosure concerns with 12 -items vs 8 -items, negative self-image with 9 -items vs 8 -items, and concerns with public attitudes with 12 -items vs 7-items respectively.

Much of the literature on HIV-related stigma emerged from queries into characterizing and ameliorating uninfected populations' stigmatizing beliefs about HIV and those who acquire HIV (Brown et al., 2003; Earnshaw et al., 2013). Castro and Farmer (2005) demonstrated a positive correlation between antiretroviral (ARV) access and stigma reduction and subsequent acceptance of HIV testing and counselling (Mbonye et al., 2013; Tsai et al., 2013). However, the literature about interventions aimed at reducing stigma towards PLHIV illustrates that interventions that provide information only without an accompanying skills building or contact with PLHIV based component are less effective in decreasing stigma and increasing tolerance towards PLHIV (Brown et al., 2003). This literature has demonstrated that while there tends to be a modest reduction in stigma towards PLHIV and willingness to get tested in the short term, there is limited longitudinal data on whether or not this is maintained. Furthermore, participants' fear around acquiring HIV and anxiety around testing remained high (Brown et al., 2003).

The enactment and experience of stigma is achieved through triggers that allow individuals to label others or their selves with the devalued marker (Earnshaw et al., 2013; Holezemer et al., 2006). Alonzo and Reynolds (1995) note that in the prestigma stage, individuals are making decisions around HIV testing through their fears around HIV, HIV testing, and what it means to be at risk for HIV. Acquisition of HIV creates a dissonant identity where one is forced to renegotiate their beliefs around what it means to be HIV positive (Alonzo \& Reynolds, 1995; Earnshaw et al., 2013). While HIV-related stigma operates similarly among the general population and PLHIV in terms of its inhibitory effect on HIV testing, accessing and/ or maintenance of HIV-related care, acquirement of HIV creates a different context for engaging with HIV-related stigma because the fears around HIV seropositive status are no longer existing as external to the individual and the diagnosis serves as new trigger point (Chesney \& Smith, 1999; Earnshaw et al., 2013; Holzemer et al., 2007; Simbayi et al., 2007). As such, combating the HIV epidemic and decreasing HIV-related stigma requires moving beyond understanding and combating HIVrelated stigma primarily within the general population towards understanding and combating PLHIV's experience with HIV-related stigma (Brown et al., 2003; Earnshaw \& Chaudoir, 2009). Characterizing stigma operating at the internalized level is of particular importance not only for the ways HIV-related stigma impacts 
on the health of PLHIV but also because as demonstrated within a South African context, the general adult population harbours less negative views about HIV when compared to the internalized stigma experienced by PLHIV (Kalichman et al., 2007). Research on the HIV-related internalized stigma experienced by PLHIV demonstrated that most PLHIV experience some level of internalized stigma (Lee et al., 2002; Li \& Sheng, 2014; Onyebuchi-iwudibia \& Brown, 2014; Sekoni, Obidike, \& Balogun, 2012; Simbayi et al., 2007; Tsai, 2015). In his review of data compiled within 12 subSaharan countries, Tsai (2015) noted that the prevalence of HIV-related internalized stigma ranged between $14-26 \%$ in most of the studied countries. Higher levels of HIV-related internalized stigma has been found to be correlated with increased symptoms of cognitive-affective depression, anxiety, hopelessness, and lower selfesteem (Earnshaw et al., 2013; Lee et al., 2002; Simbayi et al., 2007; OnyebuchiIwudibia et al., 2014). Simbayi et al. (2007) demonstrated that internalized stigma accounted for the greatest variance in the assessed symptoms of depression when compared to other correlates such as health status, social support, and substance use. According to Lee et al. (2002), high levels of internalized stigma significantly predicted depression, anxiety, and hopelessness even when other correlates of psychological wellbeing such as demographics (race, gender, sexual orientation) health status, level of grief, and styles of coping with illness were controlled.

High levels of HIV-related internalized stigma have also been correlated with reporting of higher HIV-related symptoms, greater days of HIV-related care gaps, greater ARV non-adherence, and reporting of poorer general health. Increased experience of HIV-related internalized stigma has been found to be associated with lower acceptance of one's HIV seropositive status, higher levels of grief, greater substance use, less perceived social support and engagement in more passive problem solving (Earnshaw et al., 2013; Kingori et al., 2012; Lee et al., 2002; Simbayi et al., 2007). HIV-related internalized stigma also fosters a context that increases PLHIV's sensitivity to enacted and anticipated HIV-related stigma. Similarly to enacted and anticipated HIV-related stigma, internalized stigma has been linked to greater determent from seeking HIV-related treatment, avoidance of PMTCT programs, and decreased rates of disclosure (Kingori et al., 2012; Rahangdale et al., 2010; Tsai et al., 2013). Tsai et al. (2013) demonstrated that while individuals with higher levels of internalized stigma were less likely to disclose their seropositive status to all types of contacts, rates of non-disclosure increased with social distance. The results have been mixed in terms of what demographic factors confer a greater level of internalized stigma. Some studies have found no relationship between levels of experienced internalised stigma and race, gender, or income level (Hasan et al., 2012; Sekoni et al, 2012, Li et al., 2014), but many studies have shown some level of association between internalized stigma and demographics (Hasan et al., 2012). A study by Loutfy et al. (2012), found that in Canada, women and non-white identity was associated with higher levels of generalized and internalized stigma. In his study involving sub-Saharan PLHIV, Tsai et al. (2015) noted that PLHIV from 
poorer households were at more than twice the risk of experiencing internalized stigma when compared to their counterparts from the least poor households. This study also demonstrated that PLHIV who completed higher levels of education experienced lower rate of internalized stigma (Kalichman et al., 2005; Li et al., 2014; Tsai et al., 2015). Sekoni et al. (2012) went on to show that the association between higher education and lower levels of personalised and disclosure stigma were statistically significant. While the literature on effective modes of reducing HIV-related internalized stigma among PLHIV is limited, PLHIV with lower rates of internalized stigma tend to be older, have longer time frame of HIV diagnosis, and identify more as lesbian, gay, bisexual, and/or transgender (LGBT) (Lee et al., 2002; Loutfy et al., 2012 Rao et al., 2012).

Women living with HIV are particularly vulnerable (Bell, Mthembu, O'Sullivan, \& Moody, 2007; Campbell, Nair, \& Maimane, 2006; Lekas, Siegel, \& Schrimshaw, 2006; Matseke et al., 2016; Mwaura, 2008; Rankin, Brennan, Schell, Laviwa, \& Rankin, 2005; Sangaramoorthy et al., 2017; Yuh et al., 2014); especially those from low resource countries (Avert, 2018b; Bos et al., 2008; Magadi, 2011; Mwaura, 2008; Tsai, 2015). Women's vulnerability to stigma is multifactorial and at its core compounded by their gendered positionality in society and the "volitional acquirability" of HIV (Carr \& Gramling, 2004; V. A. Earnshaw et al., 2013; V. Earnshaw, Bogart, Dovidio, \& Williams, 2015; Sangaramoorthy et al., 2017). The disruptiveness of HIV-related stigma on women's interpersonal relationships potentiates their vulnerability (Crandall \& Moriarty, 1995). Women living with HIV are vulnerable to enacted, anticipated, and internalised stigma (Campbell et al., 2006; Makin et al., 2008; Sangaramoorthy et al., 2017). Women in sub-Saharan Africa account for the majority of the HIV/ AIDS burden in the region. As such, they are confronted with perceived HIV-related stigma at much higher rates than men (Magadi, 201; Mwaura, 2008). The available literature on stigma among HIV-positive individuals demonstrates higher rates of stigma among HIV-positive individuals when compared to the larger community and variability in the gendered prevalence of such stigma (Kako \& Dubrosky, 2013; Kalichman, et al., 2005; Simbayi et al., 2007). Simbayi and colleagues (2007) demonstrated that men experienced higher rates of reported stigma compared to women. However, although depression was positively correlated with stigma, women experienced higher rates of depression than men (Simbayi et al., 2007). In contrast, Mugoya and Ernst (2014) found that when compared to their male counterparts, HIV-positive women were more likely to endorse higher rates of stigma and to experience more severe levels of stigma.

One mode through which HIV-positive women are more vulnerable to stigma and the internalisation of stigma, is through the idea of intersectionality (Carr \& Gramling 2004; Earnshaw et al., 2013). Some of the literature postulates and demonstrates that an HIV diagnosis compounds the societal inequities and associated stigma already tied to their raced, classed, and gendered identities (Kako \& Dubrosky, 2013; 
Lekas et al., 2006; Mwaura, 2008; Sandelowski et al., 2004; Sangaramoorthy et al., 2017). Campbell and colleagues (2006) note three main driving forces of maintaining stigma among women: (i) fear of being infected with HIV in the setting of high rates of infections, (ii) poverty, and (iii) stigmatisation of sexuality, particularly women's sexuality. Consequently, women's particular vulnerability to HIV-related stigma is mediated by the discourse around notions of who gets infected with HIV and the idea that women's acquirement of HIV is a result of behaviours of "immoral" womanhood such as promiscuity and/or intravenous drug use (Lekas et al., 2006; Sangaramoorthy et al., 2017). Additionally, at the intersection of womanhood and poverty, HIV-positive women's vulnerability to experiencing stigma is shaped by societal disapproval of mothering in context of poverty and/or risk of transmitting HIV to a child (Sandelowski et al., 2004). According to Sangaramoorthy and colleagues (2017), HIV-positive women revealed a sense of shame around having their decisions for motherhood in the context of disclosed HIV being framed as immoral.

HIV-related stigma among HIV-positive women has been shown to be tied to lower rates of disclosure and lower engagement in HIV-related care (Crandall \& Moriarty, 1995; Makin et al., 2008; Medley et al., 2004; Ramlagan et al., 2018; Sangaramoorthy et al., 2017). Although numerous studies have demonstrated the modes in which HIV-positive women are vulnerable to experiencing stigma and the association with negative health outcomes, there remains a gap in our knowledge of how stigma is experienced by HIV-positive pregnant women and factors that predict the level and type of stigma they experience (Sangaramoorthy et al., 2017; Sandelowski et al., 2009). This knowledge gap is particularly apparent for those who are pregnant and living in resource-limited communities (Sangaramoorthy et al., 2017; Sandelowski et al., 2009).

Given the varied and profound ways in which HIV-related stigma, particularly internalized stigma, affects the lives of PLHIV and their ability to access and maintain HIV-related care it becomes imperative to understand how that affects pregnant HIV positive women as they navigate through the added layers of negotiating their socially constructed roles of womanhood and motherhood (Earnshaw et al., 2013; Fife \& Wright, 2000; Kingori et al., 2012; Lee et al., 2002; Simbayi et al., 2007). As we aim to improve maternal uptake and adherence to PMTCT protocols, there is a need for a multifocal mode of engaging the challenges that hinder women's abilities to fully participate in their care and that of their foetus.

\section{Significance of the study}

Characterising stigma operating at the perceived level is of particular importance not only for the ways HIV-related stigma impacts on the health of People Living with HIV (PLHIV), but also because, as demonstrated within a South African context, the general adult population harbours less negative views about HIV when compared to 
the perceived stigma experienced by PLHIV (Kalichman et al., 2005; Simbayi et al., 2007). HIV-related stigma has been linked to greater determent from seeking HIVrelated treatment, avoidance of prevention of mother to child transmission (PMTCT) programs, and decreased rates of disclosure (Kingori et al., 2012; Rahangdale et al., 2010; Tsai et al., 2013).

\section{Goal of the study}

This study aimed to examine HIV-related correlates of perceived HIV-related stigma among HIV-positive pregnant women in rural Mpumalanga province, South Africa. It sought to address the following questions:

- What are the personal characteristics and HIV factors that predict experience of perceived HIV-related stigma?

- What are the roles of intimate partner violence (IPV) and male involvement in the experience of perceived HIV-related stigma?

- How are levels of depressive symptoms related to perceived HIV-related stigma?

\section{Methodology}

\section{Study design and population}

Participants were 673 HIV positive pregnant black African women attending one of 12 randomly selected antenatal community health centres (CHC). The methods of this survey have been reported in detail elsewhere (Jones et al., 2014). Briefly, this study is part of the larger Protect Your Family (PYF) randomised controlled trial conducted among HIV positive, less than six months pregnant, black African women, attending in 12 community health centres (CHCs) in Nkangala and Gert Sibande districts in Mpumalanga province, South Africa (Jones et al., 2014). The cross-sectional data we report on were gathered between April 2014 and March 2015.

\section{Procedures}

Ethical approval was granted by the University of Miami Miller School of Medicine Institutional Review Board (IRB ID: 20130238 (CR00006122)) as well as the South African Human Sciences Research Council Research Ethics Committee with Protocol No REC 4/21/08/13. As the study was being conducted in Mpumalanga Province, study approval was also obtained from the Mpumalanga Provincial Government, Department of Health.

Participants were included only if they were 18 years or older, were HIV positive, were less than six months pregnant, not part of the PYF study at another study site, having a partner (not necessarily the father of the foetus), and willing to participate in this study. Once consented, participants completed the interview on Audio Computer-Assisted Self-Interview (ACASI) technology (NIMH Multisite 
HIV/STD Prevention Trial for African American Couples Group, 2008). Interviews were carried out either in English, seSotho or isizulu. For completing the baseline interview, participants were given R50.00 (South African Rands) or US\$4.72.

\section{Measures}

\section{Measurements of perceived internalized stigma}

Respondents were asked to respond to the 40-item perceived internalized stigma scale (Berger et al., 2001; Lindberg et al., 2014). The 40-items in the scale are statements that are answered with a 4-point Likert scale (strongly disagree, disagree, agree and strongly agree). Statements in the scale include "Having HIV makes me feel unclean," "I regret having told some people that I have HIV," "Having HIV in my body is disgusting to me," etc (See Table 2). Cronbach's Alpha reliability for the 40item scale was $\alpha=.96$.

\section{Additional instruments}

Questions regarding socio-demographic characteristics included age at last birthday; educational attainment; relationship status; employment status; income per month in South African Rand (ZAR) where the South African Government child grant was R310.00 (UD\$29.27) during the study period; number of children; if this pregnancy was unplanned; and alcohol usage of more than 2 drinks on at least one occasion in the previous four weeks.

HIV-related questions included whether the HIV diagnosis was made during their current pregnancy; whether the participant has any HIV-positive children; partners' HIV status and male involvement i.e. whether their male partner knew what occurred during in the antenatal clinic visit. ARV usage was assessed using the seven day recall Visual Analog Scale (Giordano et al., 2004) where participants were recorded as "adherent" (coded 1 ) if they took all their medication on all seven days and as " 0 " if they missed any of their medication during the past seven days (Ramlagan et al., 2018). Intimate partner violence was assessed utilising the 18item Conflict Tactics Scale 18 (CTS-18) (Straus, 1979, Straus et al., 1979). A higher score on the scale is indicative of increased IPV. Cronbach's Alpha reliability for the CST-18 was $\alpha=.84$. The 9-item AIDS-Related Stigma Scale (ARSS) (Kalichman et al., 2005) was utilized. Scores on the scale range from 0 to 8 , where higher scores indicate greater levels of stigma. Cronbach's Alpha reliability for the ARSS was $\alpha=$ .73. Depression was assessed utilising the 10-item Edinburgh Postnatal Depression Scale 10 (EPDS-10) (Cox et al., 1987). Scores ranged from 0 to 30, where the higher the score, the more the likelihood of depression. Validated cut-off score of 12 for South African populations was utilized (Lawrie, 1998). Cronbach's Alpha reliability for the EPDS-10 was $\alpha=.66$. 


\section{Data analysis}

Software SPSS, version 24.0 (I.B.M Corp, 2016) was used for data analyses. Frequencies, means and cross tabulations were calculated to describe the sample. Reliability tests were also done on the ARSS, EPDS, and IPV scales. Factor analysis was done to the Berger et al (2001) HIV stigma scale and variability was described. Reliability tests were done on each of the four factors, namely personalized stigma, concerns with public attitude, disclosure concerns, and negative self-image. Univariate and multivariate logistic regression were used to investigate associations between the four factors of internalized stigma and socioeconomic, HIV related, and behavioural variables. Associations were considered significant at $P<0.05$. All statistically significant variables in the univariate analyses were included in the multivariate logistic regression. Multi-collinearity was tested.

\section{Results}

The mean age of the 673 HIV positive pregnant participants were 28 years old $(S D=5.73)$. Of these participants, the majority $(71.5 \%)$ did not complete grade 12 (matric) school (see Table 1). A high third of the participants reported that their monthly household income was less than R310 which was the South African government child grant amount during the study time period. Off all respondents, $20 \%$ stated that they experienced mild to severe physical violence from their intimate partners, $41 \%$ experienced AIDS related stigma and $42 \%$ experienced no male partner involvement in this pregnancy (see Table 1). Interestingly, half of the HIV positive pregnant experienced low depression and just over half were diagnosed as HIV positive during this pregnancy.

Table 1: Sample demographics

\begin{tabular}{ll} 
Variable & $\mathbf{N}(\%)$ \\
\hline All & $673(100)$ \\
\hline Age (m=28.39, SD=5.73) & \\
\hline Educational attainment & $192(28.5)$ \\
\hline Grade 12 or more & $481(71.5)$ \\
Grade 11 and less & \\
\hline Relationship status & $419(62.3)$ \\
\hline Not living together & $254(37.7)$ \\
Living together & \\
\hline Employment status & $527(78.3)$ \\
\hline Not employed & $146(21.7)$ \\
Employed, Volunteer or Student & \\
\hline Income (ZAR)per month & $221(32.8)$ \\
\hline$<$ R310 & $452(67.2)$ \\
\hline R311 or more &
\end{tabular}


Table 1: Sample demographics (cont...)

\begin{tabular}{|c|c|}
\hline Variable & $N(\%)$ \\
\hline All & $673(100)$ \\
\hline \multicolumn{2}{|l|}{ Age $(m=28.39, S D=5.73)$} \\
\hline \multicolumn{2}{|l|}{ Number of children } \\
\hline None & $139(20.7)$ \\
\hline One or more & $534(79.3)$ \\
\hline \multicolumn{2}{|l|}{ Unplanned pregnancy } \\
\hline No & $317(47.1)$ \\
\hline Yes & 356(52.9) \\
\hline \multicolumn{2}{|c|}{ Alcohol use of more than 2 drinks on at least on one occasion in the past 4 weeks } \\
\hline No & $581(86.3)$ \\
\hline Yes & $92(13.7)$ \\
\hline \multicolumn{2}{|l|}{ Diagnosed with HIV in this pregnancy } \\
\hline No, before & $308(45.8)$ \\
\hline Yes & $365(54.2)$ \\
\hline \multicolumn{2}{|l|}{ Has HIV positive children } \\
\hline No or do not know & $506(75.2)$ \\
\hline Yes & $28(4.2)$ \\
\hline \multicolumn{2}{|l|}{ VAS adherence } \\
\hline non adherent & 210(31.2) \\
\hline $100 \%$ adherent & $463(68.8)$ \\
\hline \multicolumn{2}{|l|}{ HIV positive partner } \\
\hline No or do not know & $506(75.2)$ \\
\hline Yes & $167(24.8)$ \\
\hline \multicolumn{2}{|l|}{ Intimate partner violence } \\
\hline No mild or No severe physical violence & $541(80.4)$ \\
\hline Mild or severe physical violence & 132(19.6) \\
\hline \multicolumn{2}{|l|}{ AIDS related stigma } \\
\hline No & $400(59.4)$ \\
\hline Yes & $273(40.6)$ \\
\hline \multicolumn{2}{|l|}{ Depression } \\
\hline No/Low & $345(51.3)$ \\
\hline High & $328(48.7)$ \\
\hline \multicolumn{2}{|l|}{ Male involvement } \\
\hline No & $281(41.8)$ \\
\hline Yes & $392(58.2)$ \\
\hline
\end{tabular}

Exploratory factor analysis of the perceived internalized stigma constructs yielded an initial 6 factors solution (data not shown). The Kaiser-Meyer-Olkin measure of 
sampling adequacy received a test score of .961 and the Bartlett's test of sphericity received a significance of .000 indicating that the sampling is adequate and that a factor analysis may be useful with the data (IBM Knowledge Center, 2018). Items with a loading of $>.3$ were included in the final version. The scree plot of the initial factor analysis, the Berger et al. (2001) and Lindberg et al. (2014) analysis, and the presence of a simpler structure (Berger et al., 2001) were utilised in deciding on how many factors to extract. A four factor solution resulted from the above analyses and included personalised stigma (17 items), concerns with public attitudes about people with HIV (7 items), disclosure concerns (6 items), and negative self-image (10 items) (see Table $2 \& 4$ ). Where loadings appeared in more than one component, the stronger of the two loadings was taken.

Table 2: Factor Analysis loadings of internalized stigma

\begin{tabular}{|c|c|c|c|c|}
\hline \multirow{3}{*}{ Items } & \multicolumn{4}{|c|}{ Component } \\
\hline & 1 & 2 & 3 & 4 \\
\hline & $\begin{array}{l}\text { Personalized } \\
\text { stigma }\end{array}$ & $\begin{array}{l}\text { Concerns } \\
\text { with public } \\
\text { attitude }\end{array}$ & $\begin{array}{l}\text { Disclosure } \\
\text { concerns }\end{array}$ & $\begin{array}{l}\text { Negative } \\
\text { self-image }\end{array}$ \\
\hline $\begin{array}{l}\text { People don't want me around their children } \\
\text { once they know I have HIV }\end{array}$ & 0.871 & & & \\
\hline $\begin{array}{l}\text { People I care about stopped calling after } \\
\text { learning I have HIV }\end{array}$ & 0.845 & & & \\
\hline $\begin{array}{l}\text { People have physically backed away from me } \\
\text { when they learn I have HIV }\end{array}$ & 0.844 & & & \\
\hline $\begin{array}{l}\text { People have told me that getting HIV is what I } \\
\text { deserve for how I lived my life }\end{array}$ & 0.828 & & & \\
\hline I have lost friends by telling them I have HIV & 0.801 & & & \\
\hline $\begin{array}{l}\text { Some people avoid touching me once they } \\
\text { know I have HIV }\end{array}$ & 0.759 & & & \\
\hline $\begin{array}{l}\text { Some people act as though it's my fault I have } \\
\text { HIV }\end{array}$ & 0.740 & & & \\
\hline $\begin{array}{l}\text { Some people close to me are afraid others will } \\
\text { reject them if it becomes known that I have HIV }\end{array}$ & 0.736 & & & \\
\hline $\begin{array}{l}\text { People who know I have HIV tend to ignore my } \\
\text { good points }\end{array}$ & 0.735 & & & \\
\hline $\begin{array}{l}\text { People seem afraid of me once they learn I } \\
\text { have HIV }\end{array}$ & 0.714 & & & \\
\hline $\begin{array}{l}\text { I have stopped socializing with some people } \\
\text { because of their reactions to my having HIV }\end{array}$ & 0.695 & & & \\
\hline $\begin{array}{l}\text { As a rule, telling others that I have HIV has been } \\
\text { a mistake }\end{array}$ & 0.575 & & & \\
\hline $\begin{array}{l}\text { I have told people close to me to keep the fact } \\
\text { that I have HIV a secret }\end{array}$ & 0.521 & & & \\
\hline $\begin{array}{l}\text { I have been hurt by how people reacted to } \\
\text { learning I have HIV }\end{array}$ & 0.508 & & & \\
\hline $\begin{array}{l}\text { When people learn you have HIV, they look for } \\
\text { flaws in your character }\end{array}$ & 0.498 & & & \\
\hline I regret having told some people that I have HIV & 0.497 & & & \\
\hline $\begin{array}{l}\text { Some people who know I have HIV have grown } \\
\text { more distant }\end{array}$ & 0.313 & & & \\
\hline
\end{tabular}


Table 2: Factor Analysis loadings of internalized stigma (cont...)

\begin{tabular}{|c|c|c|c|c|}
\hline \multirow{3}{*}{ Items } & \multicolumn{4}{|c|}{ Component } \\
\hline & 1 & 2 & 3 & 4 \\
\hline & $\begin{array}{l}\text { Personalized } \\
\text { stigma }\end{array}$ & $\begin{array}{c}\text { Concerns } \\
\text { with public } \\
\text { attitude }\end{array}$ & $\begin{array}{l}\text { Disclosure } \\
\text { concerns }\end{array}$ & $\begin{array}{l}\text { Negative } \\
\text { self-image }\end{array}$ \\
\hline I feel guilty because I have HIV & & 0.754 & & \\
\hline $\begin{array}{l}\text { In many areas of my life, no one knows that I } \\
\text { have HIV }\end{array}$ & & 0.710 & & \\
\hline $\begin{array}{l}\text { People's attitudes about HIV make me feel } \\
\text { worse }\end{array}$ & & 0.665 & & \\
\hline Telling someone I have HIV is risky & & 0.639 & & \\
\hline I work hard to keep my HIV a secret & & 0.549 & & \\
\hline $\begin{array}{l}\text { People with HIV lose their jobs when their } \\
\text { employers find out }\end{array}$ & & 0.416 & & \\
\hline $\begin{array}{l}\text { I never feel ashamed of having HIV (Reverse } \\
\text { scored) }\end{array}$ & & 0.383 & & \\
\hline I am very careful who I tell that I have HIV & & & -0.670 & \\
\hline $\begin{array}{l}\text { Most people are uncomfortable around } \\
\text { someone with HIV }\end{array}$ & & & -0.601 & \\
\hline $\begin{array}{l}\text { I never feel the need to hide the fact that I have } \\
\text { HIV (Reverse scored) }\end{array}$ & & & -0.584 & \\
\hline $\begin{array}{l}\text { I worry that people may judge me when they } \\
\text { learn I have HIV }\end{array}$ & & & -0.558 & \\
\hline $\begin{array}{l}\text { I worry that people who know I have HIV will } \\
\text { tell others }\end{array}$ & 0.324 & & -0.450 & \\
\hline $\begin{array}{l}\text { Since learning I have HIV, I worry about people } \\
\text { discriminating against me }\end{array}$ & & & -0.447 & \\
\hline Having HIV makes me feel unclean & & & & -0.734 \\
\hline $\begin{array}{l}\text { Most people believe that a person who has HIV } \\
\text { is dirty }\end{array}$ & & & & -0.713 \\
\hline $\begin{array}{l}\text { Having HIV makes me feel that I am a bad } \\
\text { person }\end{array}$ & & & & -0.683 \\
\hline $\begin{array}{l}\text { Most people think that a person with HIV is } \\
\text { disgusting }\end{array}$ & & & & -0.675 \\
\hline $\begin{array}{l}\text { Since learning that I have HIV, I feel set apart } \\
\text { and isolated from the rest of the world }\end{array}$ & & & & -0.673 \\
\hline $\begin{array}{l}\text { It is easier to avoid new friendships than worry } \\
\text { about telling someone that I have HIV }\end{array}$ & & & & -0.604 \\
\hline People with HIV are treated like outcasts & & & & -0.522 \\
\hline $\begin{array}{l}\text { Most people with HIV are rejected when others } \\
\text { find out }\end{array}$ & & & -0.505 & -0.509 \\
\hline $\begin{array}{l}\text { I feel I am not as good a person as others } \\
\text { because I have HIV }\end{array}$ & & 0.345 & & -0.496 \\
\hline Having HIV in my body is disgusting to me & & & & -0.418 \\
\hline
\end{tabular}


Within these four components, namely personalised stigma, concerns with public attitude, disclosure concerns and negative self-image, correlation coefficients of .3 and above are observed. The four components explain a total of $53.69 \%$ of the variance (see Table 3). All bivariate correlations among the four factors go in a positive direction (see Table 3).

Table 3: Correlation matrix and variance of the four dimensions of internalised stigma

\begin{tabular}{llrrrr}
\multicolumn{7}{c}{$\mathbf{1}$} & $\mathbf{2}$ & $\mathbf{3}$ & $\mathbf{4}$ \\
\hline 1 & Personalized stigma & 1 & & & \\
\hline 2 & Concerns with public attitude & $.489^{* *}$ & 1 & & \\
\hline 3 & Disclosure concerns & $.676^{* *}$ & $.524^{* *}$ & 1 & \\
\hline 4 & Negative self-image & $.699^{* *}$ & $.628^{* *}$ & $.682^{* *}$ & 1 \\
\hline & & & & 1.63 & 1.45 \\
\hline Eigenvalues & 15.53 & 2.87 & 4.08 & 3.63 \\
\hline$\%$ Variance & 38.81 & 7.17 & 673 & 673 \\
\hline $\mathrm{N}$ & 673 & 673 & &
\end{tabular}

The Cronbach's Alpha for each of the four subscales was between $\alpha=.79$ to $\alpha=.94$ providing evidence for the internal consistency of the resulting subscales (see Table 4).

Table 4: Descriptive of the four Internalised Stigma factors $(N=673)$

\begin{tabular}{lrrrrr} 
Factor Name & Items & M(SD) & Skewness* & Kurtosis** & Alpha \\
\hline Personalized stigma & 17 & $35.17(10.38)$ & 0.209 & 0.005 & 0.944 \\
\hline Concerns with public attitude & 7 & $15.98(4.56)$ & 0.140 & -0.077 & 0.794 \\
\hline Disclosure concerns & 6 & $14.36(4.00)$ & -0.219 & 0.007 & 0.819 \\
\hline Negative self-image & 10 & $21.14(6.54)$ & 0.316 & 0.015 & 0.894 \\
\hline *Std. Error=0.094 & & & & & \\
$* *$ Std. Error=0.188 & & & & &
\end{tabular}

Logistic regression was carried out to predict associations that would lead to the likelihood of participants experiencing internalised stigma. The univariate logistic regression and multivariate logistic regression are presented in Table 5. In terms of personalized stigma, the univariate logistic regression shows that those respondents that have Grade 11 or less of schooling, those who receive a monthly household income of less than R310, those who experience IPV, those who experience more AIDS related stigma and those who have no male involvement in their current pregnancy were more likely to experience personalised stigma. All variables that were found to be statistically significant in the univariate analysis were put into a multivariate logistic regression. The multivariate logistic regression on personalized 
stigma found significant unique associations with not completing schooling i.e. Grade 11 or less, income of less than R310, those who experience IPV, and those that have no male involvement in their current pregnancy.

The univariate and multivariate logistic regressions found statistically significant associations with the internalized stigma component of concerns with public attitude and those who receive a monthly household income of less than R310, and those that have no male involvement in their current pregnancy (see Table 5). In terms of disclosure concerns, both the univariate and multivariate logistic regression found that those who receive a monthly household income of less than R310 and those that have no male involvement in their current pregnancy were more likely to experience disclosure concerns. With the final component of internalized stigma, i.e. negative self-image, the univariate logistic regression shows that those who experience IPV, those that experienced more AIDS related stigma and those that have no male involvement in their current pregnancy were more likely to experience negative self-image (see Table 5). Table 5 shows that in the multivariate logistic regression, those who experienced more AIDS related stigma and those that have no male involvement in their current pregnancy were more likely to experience negative self-image. 
Table 5: Association of demographic, socioeconomic, health, and behaviour characteristics to factors of internalized stigma

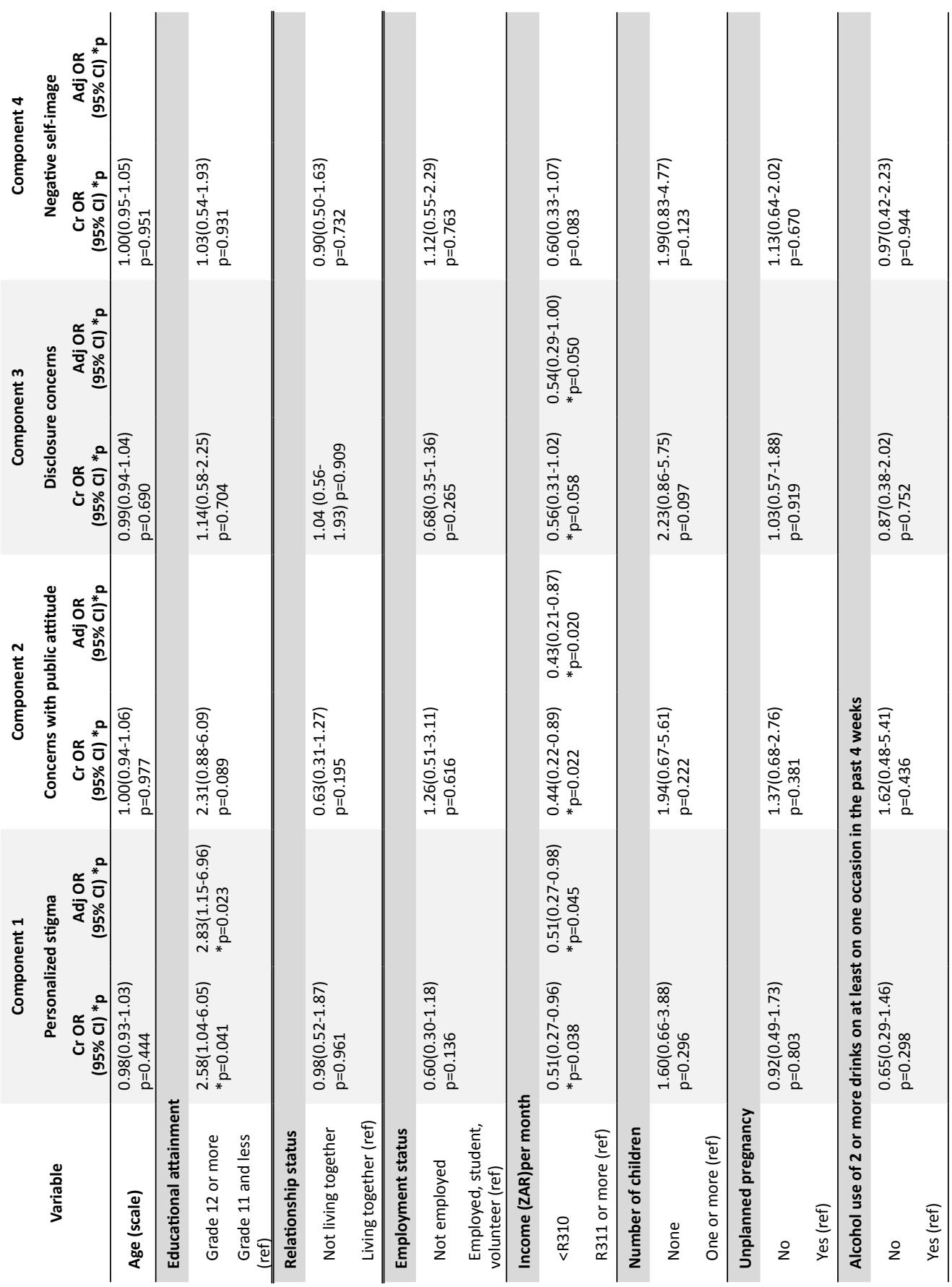


Table 5: Association of demographic, socioeconomic, health, and behaviour characteristics to factors of internalized stigma (cont...)

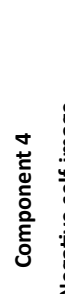

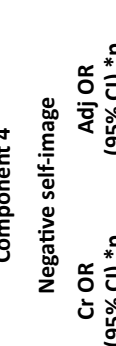

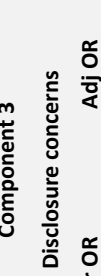

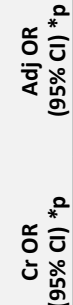

กิ

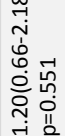

ำ

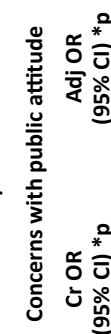

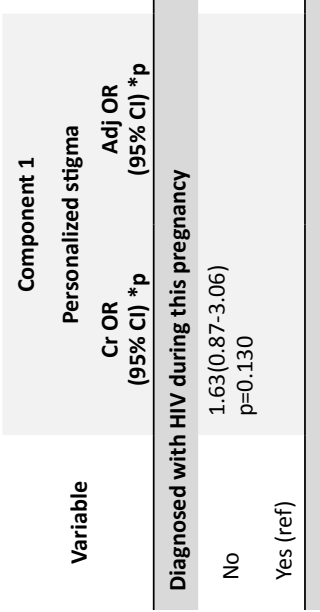

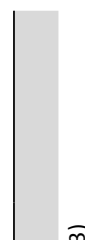
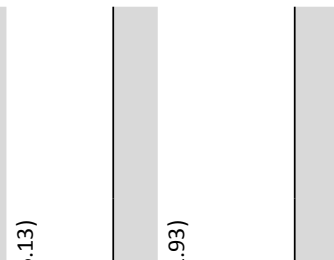

\section{กิ่}

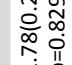

仓े

นึํ요

पे

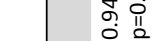

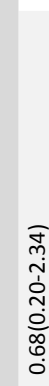

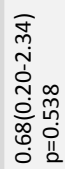

$\widehat{6}$
i.
$\dot{j}$
$\dot{4}$
0
0
0
0
0
0
0
0

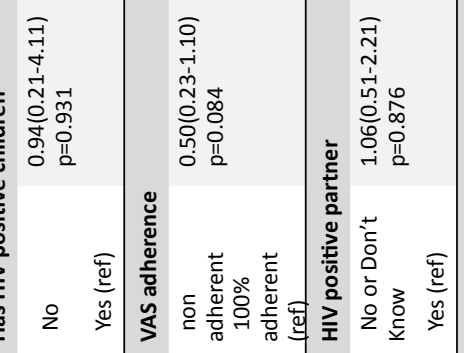

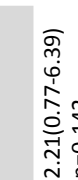

1
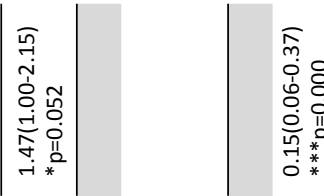

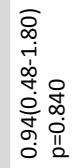

ने

要

늘워

in

जิ

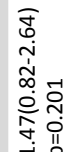

心

กิ8

임

䨍

क্口

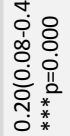

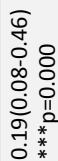

ळ

mำ

일

mit

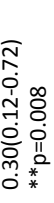

ळ.

o

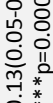

光 **

*

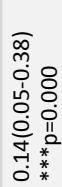

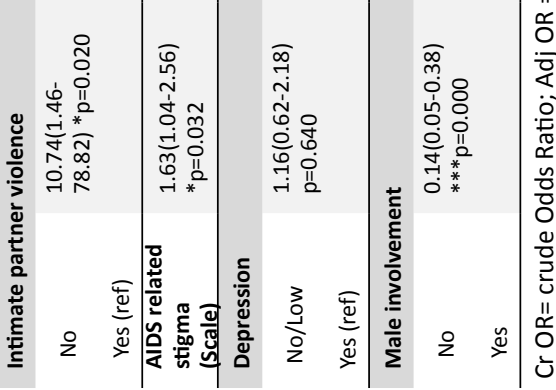




\section{Discussion}

This study explored the correlates of HIV-related internalized stigma among HIV positive pregnant women in rural Mpumalanga province, South Africa. To achieve this, the full 40-item internalized stigma scale (Berger et al., 2001) was utilized to measure four factors of stigma for the first time in a South African context. Internal consistency was shown to be high for the scale and thus generated good quality data. The exploratory factor analysis produced a four factors sequence of personalised stigma, concerns with public attitudes about people with HIV, disclosure concerns, and negative self-image. The same four factors were noted by Berger et al. (2001) in America and Lindberg et al. (2014) in Sweden yet their sequence of factors placed concerns with public attitudes about people with HIV at the end of their sequence. The number of item that made up each of the four factors were different in this study when compared to Berger et al. (2001) and Lindberg et al. (2014).

Having no male partner involvement in the pregnancy was significantly associated with experiencing all four of the HIV-related stigma factors. This significant finding leads us to deduce that positive male involvement seems critical in assisting pregnant women to potentially reduce what Scheff (1966) defines as their self-defacing beliefs and perceptions of themselves. Male involvement in PMTCT has been recognised as a priority area in the elimination of mother-to-child transmission of HIV where their positive involvement in the pregnancy can have an influence in the extended social environment of the family, can increase health seeking behaviour, can increase adherence to ARV medication as well as clinic appointments, and assist to mitigate stigma (WHO, 2012a). It is imperative to caution that, in this study, male involvement was measured by utilising only one question. Using one question as a measure of involvement in pregnancy is limiting and as such, the result, although significant must be interpreted with caution as additional in-depth research in the area of male involvement in reducing stigma among HIV-positive women is needed. Women with a low income were more likely to experience three of the four HIVrelated stigma factors. This finding is similar to the finding of Tsai and colleagues (2015), who noted that PLHIV from poorer households were at more than twice the risk of experiencing HIV related stigma. Additionally, poverty is one of the three main driving forces of maintaining stigma (Campbell et al, 2006). Poverty increases vulnerability (Avert, 2018). Furthermore, Matseke and colleagues (2016) found that poverty combined with HIV-positive status leads to additional burden in the lives of pregnant women. This burden places HIV-positive, stigmatised women at risk of being less able to adopt HIV risk-reducing behaviours (Avert, 2018) and thus perpetuating the cycle of their devalued perceptions of themselves.

IPV was significantly associated with experiencing personalised stigma. This might be explained by the fact that experiencing IPV while HIV-positive and pregnant can lead to the development of self-deprecating constructions of oneself through the 
embodiment of shame, guilt, blame, and hopelessness (Brown et al, 2003; Earnshaw et al., 2013; Rao et al., 2012). It is this acceptance and subsequent identification with the devalued construction of themselves (Kingori et al., 2012; Rao et al., 2012) that probably leads to increased perceived HIV-related stigma. This could also be related to ARSS related stigma, which was experienced by an extremely high $41 \%$ of the study population, and was only associated in the multivariable logistic regression with the stigma of negative self-image. Participants that did not complete school, were more likely to experience personalised stigma. This finding is similar to that of Sekoni, Obidike, and Balogun (2012), who found association between higher education and lower levels of personalised and disclosure stigma. As with IPV mentioned above, those with a lower education may also develop self-deprecating constructions of themselves (Brown et al., 2003; Earnshaw et al., 2013; Rao et al., 2012). This could be due to those with lower levels of education also experiencing poorer living and working conditions (Sekoni et al., 2012). Additionally, they may be less knowledgeable about adopting risk-reducing behaviours (Avert, 2018).

\section{Conclusion}

In conclusion, the Berger et al. (2001) 40-item internalized stigma scale has been shown to be a valid and reliable scale to measure the four factors of stigma in a South African context. The scale will benefit from more evaluation in rural areas and among vulnerable populations. This study reveals the ways in which higher levels of education, income, and positive partner involvement (outside of the context of IPV) serve as protective factors against perceived HIV-related stigma, at multiple layers. Improving on adult education and income generating activities can help in reducing HIV related stigma. Strategies need to be developed to encourage male partners to be positively involved in their partner's pregnancy and as such, more research is required to explore male involvement in PMTCT in the South African context. Immediate action is needed to tackle IPV and could be achieved through community-based IPV prevention interventions and support groups for both women and men. 
CHAPTER 5

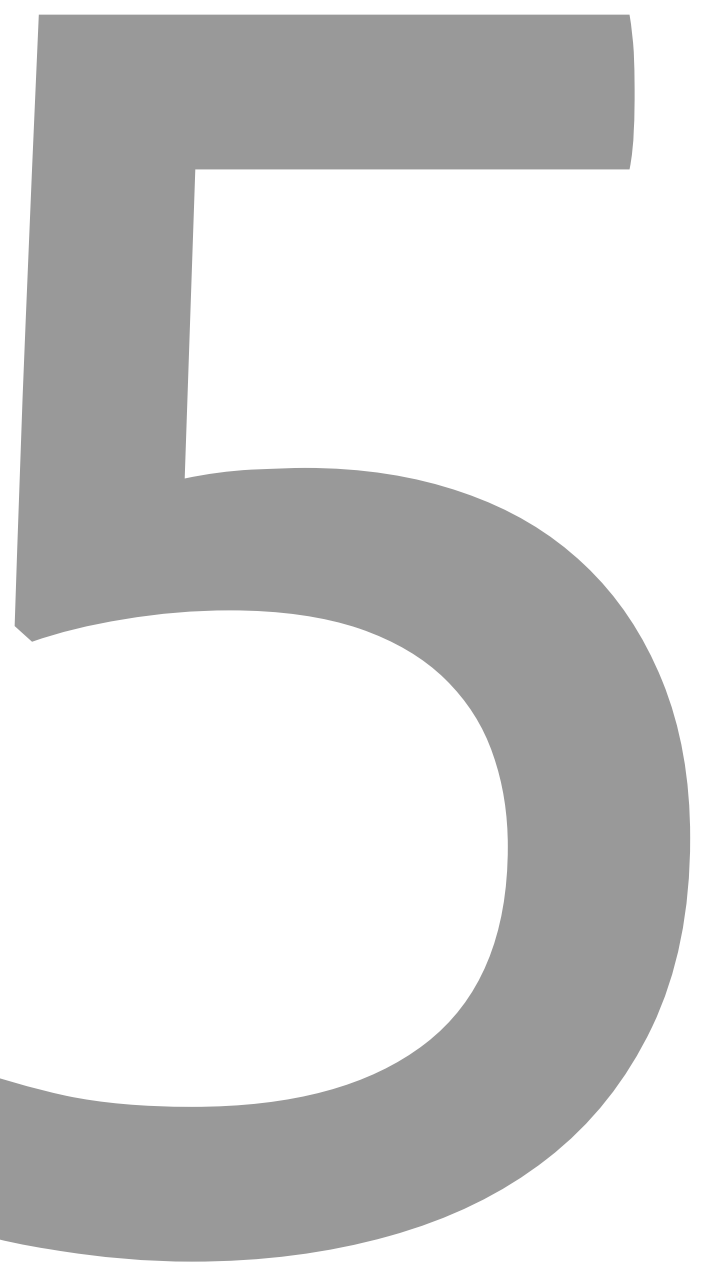




\section{SELF-REPORT AND DRY BLOOD SPOT MEASUREMENT OF ANTIRETROVIRAL MEDICATIONSAS MARKERS OFADHERENCE IN PREGNANT WOMEN IN RURAL SOUTH AFRICA}

Published as:

Alcaide, M.L., Ramlagan, S., Rodriguez, V.J., Cook, R., Peltzer, K., Weiss, S.M., Sifunda, S. \& Jones, D.L. (2017). Self-Report and Dry Blood Spot Measurement of Antiretroviral Medications as Markers of Adherence in Pregnant Women in Rural South Africa. 


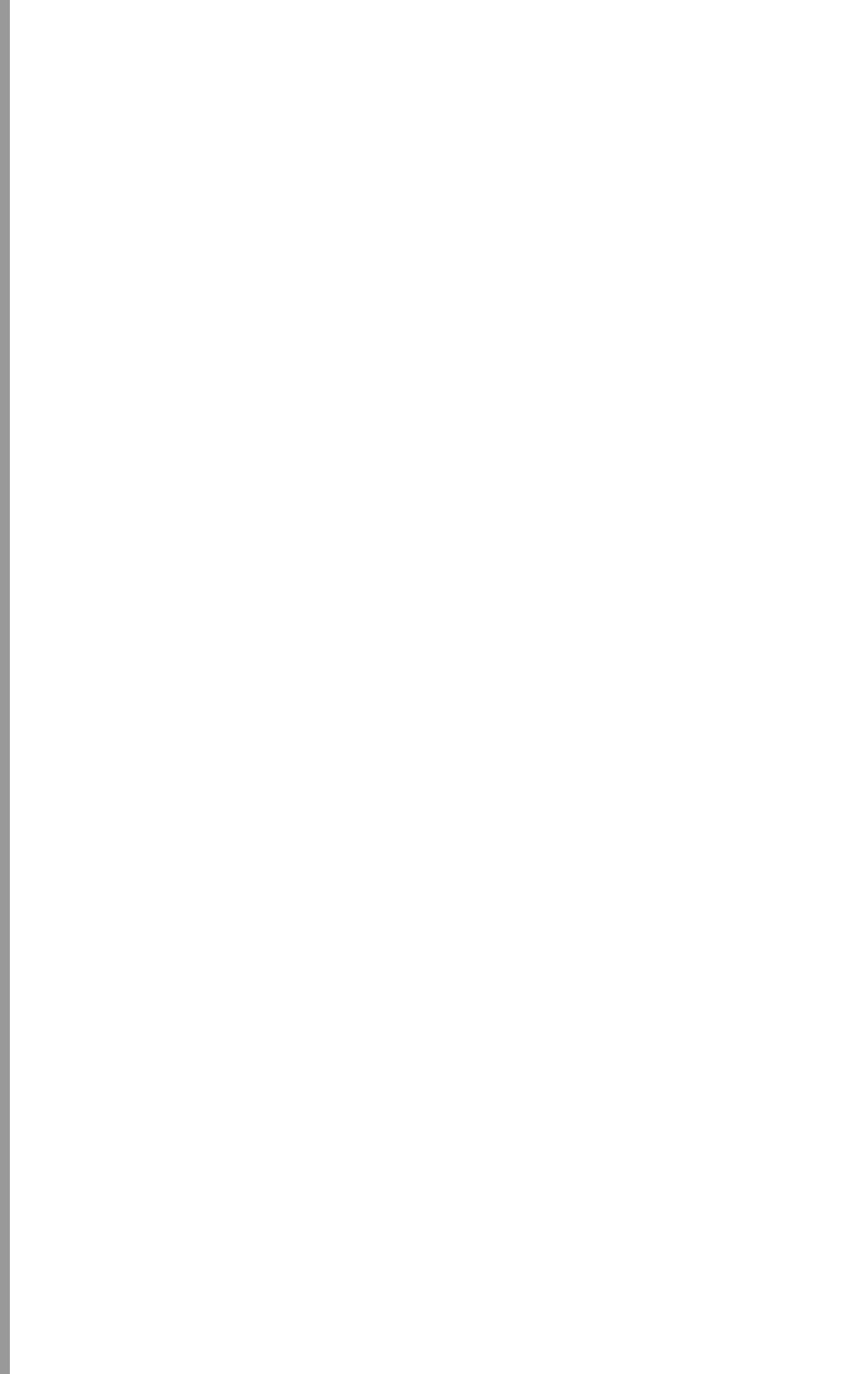




\section{Introduction}

Adherence to antiretroviral (ARV) therapy is an essential component of Prevention of Mother to Child Transmission (PMTCT) programs, as viral suppression is necessary to decrease perinatal transmission, minimize the risk of antiretroviral (ARV) drug resistance, and achieve a generation born free of HIV. South African PMTCT treatment protocols provide ARVs to expectant mothers until after the cessation of breastfeeding (option B). Currently, first-line ARV regimens for PMTCT are tenofovir (TDF) plus lamivudine (3TC) or emtricitabine (FTC) plus efavirenz (EFV), and the use of a single combination pill is recommended when possible (such as TDF + FTC + EFV combination pill or TDF + FTC combination pill)(Volk, Marcus, Phengrasamy, Blechinger, Nguyen, Follansbee, \& Hare, 2015).

In South Africa and worldwide, adherence to PMTCT among pregnant women is suboptimal. Previous research on PMTCT adherence has utilized self-report and pill count measures and found rates of ARV adherence around 61\% in South Africa, and 75\% worldwide (Nachega et al., 2012). Additionally, accurate measurement of adherence to ARV is challenging as most adherence tools utilize self-report. Validated measures of self-reported ART adherence include visual analog scales (VAS) and 4-day recall of missed medication (AIDS Clinical Trials Group Adherence scale, 4-days) but are subject to bias (Giordano et al., 2004). The use of computerized interview self-administration may reduce social desirability bias when measuring self-reported adherence using the VAS or the ACTG scale, compared to interpersonal interviews (Kalichman et al., 2009). The use of pill counts, even more advanced pill-counting strategies, e.g., electronic pill-counters track the number of openings and closings, to measure adherence to ARV decrease recall bias, but may also be inaccurate, as pills may be stored in multiple containers when unannounced pill counts occur or may purposely be discarded prior to clinic visits, providing an imprecise measurement of adherence (Bangsberg, Hecht, Charlebois, Chesney, \& Moss, 2001).

Dried blood spots (DBS) are markers of adherence that measure the presence of ARV in the blood. DBS are obtained by placing a drop of blood on a filter paper and measuring the presence of ARV. DBS are a biological measure of the presence of $A R V s$, and the absence of ARVs in the blood may indicate increased risk of perinatal transmission in PMTCT programs. Though there is no gold standard adherence measurement, DBS is superior to self-reported measures, and is a simple and reproducible measure of ARV adherence (Castillo-Mancilla, Searls, Caraway, Zheng, Gardner, Predhomme, Bushman, Anderson, \& Meditz, 2014). Although DBS have been used as markers of adherence to ARV in prevention and treatment studies, they have not previously evaluated in PMTCT programs in rural areas of South Africa, which continue to have antenatal HIV rates as high as $46 \%$ despite significant reductions in urban areas (Department of Health, 2012). This study compared the 
accuracy of two measures of self-reported adherence with DBS used to detect ARVs to estimate the accuracy of self-reported adherence among HIV-infected pregnant women in rural South Africa.

\section{Methods}

\section{Participants and Procedures}

Participants ( $n=392$ ) were HIV-infected women at week 32 of pregnancy, 18 years of age or older, having a sexual male partner, and living in rural South Africa. Women at different stages of gestation $(M=18.07, S D=5.54)$ were recruited into the baseline phase of a large randomized control trial. Three hundred and ninety two participants completed the study visit scheduled at 32 weeks of pregnancy, which included assessment of adherence using DBS. This study was conducted in 12 community health centers in Gert Sibande and Nkangala districts, Mpumalanga province, South Africa. The larger study evaluated an intervention, Protect Your Family, to improve PMTCT among pregnant HIV-infected women. Participants were recruited at week 24 of pregnancy or less, and completed assessments on adherence (self-reported adherence using the VAS and ACTG scales, and measurement of ARV by DBS) at 32 weeks of pregnancy. All participants were on ARV therapy per the South African PMTCT protocol (TDF + 3TC or FTC + EFV). Participants completed study measures in their preferred language (English, Zulu, Sotho) using an Audio Computer-Assisted Self-Interview (ACASI) to enhance disclosure and reduce bias. Prior to the onset of recruitment, all measures were translated into local languages and were adapted to the local setting as appropriate. Details of the Protect Your Family study intervention have been previously described (Jones et al., 2014). Participants were compensated with R100 (South African Rand or ZAR US\$10) for their time and transportation to the assessment.

\section{DSB collection and analysis}

At 32 weeks of pregnancy, participants were requested to provide five drops of blood obtained using a sterile lancet and a Guthrie card; participants had the option to refuse the DBS collection with no reprisals. No participants refused collection of DBS. The DBS was collected by trained nursing staff at each of the 12 study community health center and couriered to an independent University of Cape Town Division of Pharmacology laboratory blinded to study outcomes. DBS were identified using only participant ID and a laboratory barcode to maintain anonymity. DBS were tested for ARVs distributed by the South African government as first line regimens for PMTCT, all of which include some combination with TDF, EFV or 3TC. Antiretroviral drug detection was used by using liquid chromatography/tamdem mass spectrometry. 


\section{Measures}

\section{Demographic and Medical History Questionnaire}

Demographics assessed included age, educational attainment, employment status, monthly income, number of children, whether their pregnancy was planned, and whether they had two on drinks on one occasion in the past 4 weeks. Medical history questions included whether women had been diagnosed during the current pregnancy, whether they had disclosed to anyone, months since ART initiation, and the number of HIV-infected children.

\section{Partner Related Questions}

Partner related questions included whether women had disclosed to their partner, the partner was HIV infected, and whether condom was used at last sex.

\section{Male Involvement}

Male involvement was assessed using the male involvement index. In this sample, reliability for this scale was acceptable $(\alpha=0.83)$. Scores for this scale ranged from 0 to 11 , where scores indicate greater partner involvement (Byamugisha, Tumwine, Semiyaga, \& Tylleskär, 2010).

\section{Dried Blood Spot Adherence}

Levels of TDF, EFV, and 3TC levels were assessed among all participants. Cut-off values for all ARVs were undetectable if below $0.02 \mu \mathrm{g} / \mathrm{ml}$ and detectable if above $0.02 \mu \mathrm{g} / \mathrm{ml}$. Adherence was defined as either 2 or 3 drugs detected (TDF, EFV, or 3TC). As FTC was not measured and the combination of TDF + FTC in a single pill is recommended, the combination of TDF + EFV detected was also considered adherent. Nonadherence was defined as no detectable ARV, one ARV detectable, or a combination of two ARVs detectable that did not include TDF + EFV (e.g., TDF + $3 T C$ or $3 T C+E F V)$. Efavirenz has the longest half-life and levels can be detected up to a median of 48 hours after discontinuation of the medication, and although some reports indicate individual variability in the metabolism of the drug, we assume that participants who did not take a drug in the prior 3 days would have undetectable levels (Jackson, Moyle, Watson, Tjia, Ammara, Back, Mohabeer, Gazzard, \& Boffito, 2013).

\section{Visual Analog Scale Adherence}

The Visual Analog Scale (VAS) has participants rate their level of adherence on a scale of 0 (took none of my medication) to 1 (took half of my medication) to 2 (took all of my medication) for each day in the past week (Giordano et al., 2004). For the purposes of this study, only the past 3 days were scored as totals, to match the maximum number of days covered by the DBS detection window. Participant responses consistent with having missed a dose in the past 3 days were considered nonadherent, whereas participants who reported having missed zero doses were considered adherent. 


\section{AIDS Clinical Trials Group Adherence}

The AIDS Clinical Trials Group (ACTG) adherence scale has participants report how many ARV doses they had to take each day in the past 4 days. Then, for each day in the past 4 days, participants report the number of missed ARV doses of their expected number of doses (Chesney et al., 2000). To match the maximum number of days covered by the DBS detection window, if the participant reported any missed ARV doses in the past 3 Days, their response was considered nonadherent; those reporting zero missed doses in the past 3 days were considered adherent.

\section{Statistical Analyses}

Descriptive analyses were conducted to examine overall adherence and demographic characteristics of participants. Bivariate and multivariable logistic regression models were used to estimate associations with DBS ARV adherence. Only variables reaching the $p<0.05$ level of significance in bivariate logistic regression models were included in multivariable models.

An ROC (receiver operating characteristic) curve analysis was estimated to examine the performance of the VAS and the ACTG scales in identifying participants as adherent, using DBS as the gold standard. ROC curves and area under the ROC curve (AUROC) values were also calculated. AUROC values estimated the probability of identifying an adherent participant when two participants from each group (adherent and nonadherent) were selected at random. An AUROC of 0.50 suggests that the scale is no better than chance at predicting adherence, whereas an AUROC of $\geq 0.90$ is indicative of excellent performance at predicting adherence. Kappa statistics, accuracy, sensitivity, specificity, negative predictive values, and positive predictive values were calculated. Kappa statistics measured precision, intermeasure agreement between the DBS and the scales, in which a Kappa value of 0 indicated less than chance agreement, and a value of 0.99 indicated perfect agreement.

Accuracy was calculated as the number of participants truly adherent or nonadherent in the total sample. Sensitivity values were calculated as the proportion of participants categorized as adherent by ACTG/VAS and DBS (true positives) out of the total number of participants classified as adherent by DBS (true positives plus false negatives). Specificity values were calculated as the proportion of women identified as nonadherent by ACTG/VAS and DBS (true negatives) out of the total number of participants classified as nonadherent by DBS (true negatives plus false positives). The positive predictive values were calculated as the proportion of adherent women by VAS/ACTG and the DBS (true positives) out of those classified as adherent by the VAS or ACTG (true positives plus false positives). The negative predictive values were the proportion of nonadherent participants by VAS/ACTG and the DBS (true negatives) out of the total number of participants classified as nonadherent by VAS/ACTG (true negatives plus false negatives). 
Finally, exact binomial confidence limits were calculated for sensitivity, specificity, and positive and negative predictive values. $\mathrm{R}$ statistical software [caret, Epi and epiR packages] was used for statistical analyses.

\section{Results}

\section{Demographic Characteristics}

Sample demographic characteristics were available for 379 of 392 (97\%) women. Median participant age was $28 \pm 6$. Nearly three-fourths $(72 \%)$ of women had completed at least 10 years of education, $82 \%$ were unemployed, and $40 \%$ had a monthly household income of less than 310 ZAR, USD\$24. Most women (82\%) had at least one child, nearly half (46\%) planned their pregnancy, and $12 \%$ reported having more than 2 drinks in the past 4 weeks on one occasion. More women who were adherent by DBS criteria had disclosed their HIV serostatus to at least one person ( 76 vs $65 \% ; p=0.028$ ), more had their disclosed their HIV serostatus to their partner compared with nonadherent women (62 vs 49\%; $p=0.023$ ), and more reported having an HIV infected partner ( 28 vs $18 \% ; p=0.049$ ) compared to nonadherent women. No other differences emerged between DBS adherent and nonadherent women ( $p s>0.05$ ). A summary of participant demographic, HIVrelated, partner-related characteristics is presented in Table 1.

\section{Prevalence of and Associations with DBS Antiretroviral Adherence}

Using DBS, $74 \%$ of participants were classified as adherent. Among adherent women, $72 \%$ had TDF and EFV detected and $2 \%$ had all three ARVs detected. Among nonadherent women (26\%), $11 \%$ of women had none of the 3 ARVs detected, $12 \%$ had only 1 ARV detected, and $4 \%$ had two ARVs that were not TDF and EFV detected. In bivariate analyses, baseline disclosure of HIV status to anyone, disclosure of HIV status to partner, and having an HIV positive partner were associated with nonadherence at 32-weeks of pregnancy. In multivariate analyses, no associations were found. A summary of bivariate and multivariate logistic regression models is presented in Table 1. 
Table 1: Associations between sociodemographic, alcohol use, HIV related and partner related variables and non-adherence according to antiretroviral dried blood spots (DBS) $(\mathrm{N}=379)$ All Mean (SD)n (\%)

\begin{tabular}{|c|c|c|c|}
\hline & All Mean (SD) n(\%) & Unadjusted OR (Cl=95\%), $p$ & $\begin{array}{l}\text { Adjusted OR } \\
(\mathrm{Cl}=95 \%) a, p\end{array}$ \\
\hline \multicolumn{4}{|l|}{ Sociodemographics and alcohol use } \\
\hline Age & $28.69(5.71)$ & $0.98(0.94-1.02), 0.376$ & \\
\hline \multicolumn{4}{|l|}{ Educational attainment } \\
\hline$<$ Grade 10 & 74 (19.5\%) & 1.00 & \\
\hline Grade $10-11$ & $197(52.0 \%)$ & $0.86(0.48-1.54), 0.609$ & \\
\hline Grade 12 or more & $108(28.5 \%)$ & $0.57(0.57-1.12), 0.102$ & \\
\hline $\begin{array}{l}\text { Employed (reference=not } \\
\text { employed) }\end{array}$ & $69(18.2 \%)$ & $0.60(0.31-1.14), 0.119$ & \\
\hline \multicolumn{4}{|l|}{ Income (South African Rand) } \\
\hline$<310$ & $151(39.8 \%)$ & 1.00 & \\
\hline 310-949 & $105(27.7 \%)$ & $0.68(0.38-1.20), 0.181$ & \\
\hline 950 or more & $123(32.5 \%)$ & $0.74(0.43-1.26), 0.265$ & \\
\hline At least one child (reference=none) & $312(82.3 \%)$ & $0.74(0.42-1.32), 0.311$ & \\
\hline $\begin{array}{l}\text { Pregnancy unplanned } \\
\text { (ref=planned) }\end{array}$ & $206(54.4 \%)$ & $1.16(0.73-1.83), 0.536$ & \\
\hline $\begin{array}{l}\text { Whether participant has had more } \\
\text { than } 2 \text { drinks on one occasion in } \\
\text { the past } 4 \text { weeks (ref=no) }\end{array}$ & 46 (12.1\%) & $0.75(0.36-1.57), 0.447$ & \\
\hline \multicolumn{4}{|l|}{ HIV-related variables } \\
\hline $\begin{array}{l}\text { Diagnosed with HIV in this } \\
\text { pregnancy }(\text { ref=no) }\end{array}$ & $208(54.9 \%)$ & $1.06(0.67-1.68), 0.793$ & \\
\hline $\begin{array}{l}\text { Disclosure of HIV serostatus to } \\
\text { anyone (ref=no) }\end{array}$ & $278(73.4 \%)$ & $0.58(0.35-0.94), 0.029$ & $\begin{array}{l}0.74(0.36-1.50), \\
0.401\end{array}$ \\
\hline Months since ART initiation & $13.2(23.6)$ & $1.01(0.99-1.01), 0.272$ & \\
\hline $\begin{array}{l}\text { HIV infected children (ref=no or do } \\
\text { not know) }\end{array}$ & $16(5.1 \%)$ & $1.83(0.64-5.22), 0.256$ & \\
\hline \multicolumn{4}{|l|}{ Partner-related variables } \\
\hline $\begin{array}{l}\text { Disclosure of HIV serostatus to } \\
\text { partner (ref=no) }\end{array}$ & $222(58.6 \%)$ & $0.60(0.37-0.93), 0.024$ & $\begin{array}{l}0.80(0.41-1.59), \\
0.526\end{array}$ \\
\hline $\begin{array}{l}\text { HIV infected partner (ref=no or do } \\
\text { not know) }\end{array}$ & $96(25.3 \%)$ & $0.57(0.32-0.99), 0.048$ & $\begin{array}{l}0.65(0.36-1.19), \\
0.165\end{array}$ \\
\hline $\begin{array}{l}\text { Non condom use at last sex } \\
\text { (ref=yes) }\end{array}$ & $186(49.1 \%)$ & $1.11(0.70-1.75), 0.654$ & \\
\hline Male involvement index & $7.1(3.0)$ & 0.96 (0.89-1.04), 0.304 & \\
\hline
\end{tabular}

\section{VAS and ACTG Adherence}

According to the VAS, $89 \%$ of women self-reported $100 \%$ adherence; $80 \%$ were adherent by ACTG.

VAS and ACTG scales performance relative to DBS VAS relative to $D B S$

The AUROC for the VAS was 0.543 , suggesting the VAS performed poorly in predicting 
adherence by DBS. The Kappa statistic was $\mathrm{K}=0.101$, indicating slight intermeasure agreement between the VAS and the DBS. Accuracy was estimated to be 0.719 [95\% $\mathrm{Cl} 0.67,0.76]$.

VAS sensitivity was $0.907[95 \% \mathrm{Cl} 0.87,0.94]$ and specificity $0.178[95 \% \mathrm{Cl} 0.11$, 0.27 ]. The positive predictive value (PPV) for the VAS was 0.761 [ $95 \% \mathrm{Cl} 0.71,0.80$ ], and negative predictive value (NPV) 0.400 [ $95 \% \mathrm{Cl} 0.26,0.56]$.

\section{ACTG relative to DBS}

The AUROC for the ACTG was 0.538, suggesting the ACTG performed poorly in predicting adherence by DBS. The ACTG Kappa statistic was $\mathrm{K}=0.081$, indicating poor intermeasure agreement between the ACTG and the DBS. Accuracy was estimated at $0.673[95 \% \mathrm{Cl} 0.63,0.72]$.

ACTG sensitivity and specificity values were $0.818[95 \% \mathrm{Cl} 0.77,0.86]$ and 0.257 $[95 \% \mathrm{Cl} 0.18,0.35]$, respectively. The PPV was $0.760[95 \% \mathrm{Cl} 0.71,0.81]$, and NPV $0.329[95 \% \mathrm{Cl} 0.23,0.44]$.

\section{Discussion}

This study evaluated adherence to ARV among HIV-infected pregnant women in rural South Africa using DBS, factors potentially associated with DBS and ARV nonadherence, and compared the accuracy of two measures of self-reported adherence with DBS ARV adherence. DBS ARV adherence was poor, and we did not identify sociodemographic, HIV or partner related factors associated with nonadherence. Self-reported adherence was poorly associated with DBS adherence, highlighting the importance of validation of self-report with alternative methods of assessment, such as biological markers, to optimize PMTCT goals.

Despite the use of ACASI to enhance accuracy and reduce bias, participants selfreported higher levels of adherence than identified by DBS. Previous studies utilizing ARVs for pre exposure prophylaxis (PrEP) found poor agreement between self-reported adherence measures and biological assessments among women; over $80 \%$ self-reported adherence vs. less than $25 \%$ detectable ARVs (Van Damme et al., 2012). Both research and clinical settings necessitate the use of accurate adherence assessments, and results confirm that adherence by self-report methods should be validated. However, therapeutic drug monitoring in clinical practice is not the norm in sub Saharan Africa, and the use of DBS in routine management of pregnant HIV-infected women is unrealistic due to cost and delays associated with obtaining results. Facilitating a therapeutic alliance with patients that optimizes adherence and continuity of care and better correlates self-report with biological assays may require clinical strategies that clarify the importance of adherence to patients, encourage patients to take an active role in their own treatment, and increase 
accurate self-reporting.

Low levels of adherence to ARVs among pregnant sub-Saharan African women have previously been described (Nachega et al., 2012), and in this study, only three quarters of respondents in this sample were adherent by DBS. Poor adherence places infants at risk of perinatal HIV acquisition and increases the likelihood of drug resistance, progression of HIV disease, and potential transmission of HIV to sexual partners. Maximizing ARV adherence continues to present a challenge in South Africa and interventions are needed to support pregnant HIV-infected women.

This study has limitations: 1) this cross sectional study only reflects adherence at one time point during pregnancy. Although women were participating in an intervention study and adherence may have be affected by the intervention, this report evaluates cross-sectional adherence and congruence among measures, not differences by study condition; 2) DBS data presented refers to the presence of absence of ARV, and no quantification of the exact amount of drug in the blood. This may overestimate adherence as drug may be detectable but at subtherapeutic levels or women may have been adherent only a few days before DBS collection and not for longer period of time; 3 ) other adherence methods were neglected, e.g., pill count, electronic caps or other biological methods, but the VAS has been reported to provide similar adherence estimates as unannounced pill counts, and is superior to self-reported recalls of missed doses (Kalichman et al., 2009); 4) comparing 3 days self-reported adherence to EFV may not correlate with a non-detectable level as isolated reports have shown that EFV may be detectable in blood up to 2 weeks, which may have overestimated adherence (Jackson et al., 2013); 5) assessment of viral load would have provided with a measure of the potential risk of perinatal transmission; 6) DBS may be subject to social desirability bias as patients may take their ARVs prior to clinic visits if they are aware that their ARV levels will be examined. Future studies and clinical trials should include alternate biological assessments of adherence such as hair sampling to evaluate ARV uptake over longer periods (e.g., one month), and viral load measuring.

\section{Conclusions}

Findings showed that self-report measures of ARV adherence overestimate adherence when compared with biological measures, and interventions to increase adherence to ARV among pregnant women are urgently needed (Castillo-Mancilla et al., 2014). Future work should include triangulation of multiple adherence assessment methods, and compare them with biological measures, in order to inform adherence interventions in the context of PMTCT and to reduce the risk of HIV transmission to infants. 

CHAPTER 6 


\section{SELF-REPORTED LONG-TERM ANTIRETROVIRAL ADHERENCE: A LONGITUDINAL STUDY AMONG HIV INFECTED PREGNANT WOMEN IN Mpumalanga, SOUTH AFricA}

Published as:

Ramlagan, S., Rodriguez, V.J., Peltzer, K., Ruiter, R.A.C., Jones, D.L., \& Sifunda, S. (2019). Self-reported long-term antiretroviral adherence: a longitudinal study among HIV infected pregnant women in Mpumalanga, South Africa. 


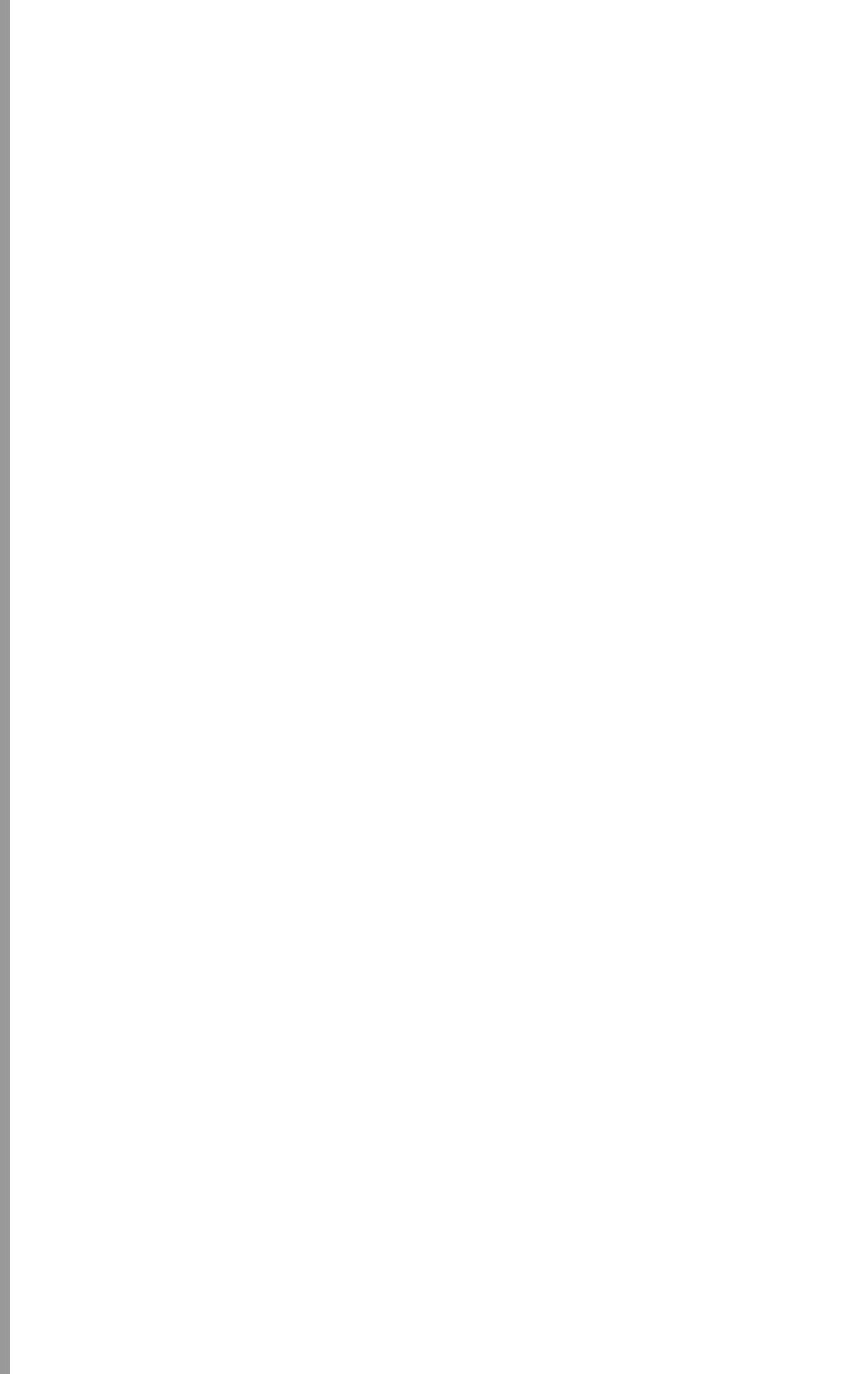




\section{Introduction}

Women who are pregnant and living with Human Immunodeficiency Virus (HIV) are recognised as part of the HIV key and vulnerable population group (SANAC, 2016; UNAIDS, 2015). During pregnancy, antiretroviral therapy (ART) is recommended for HIV infected women for viral suppression and reduction of perinatal HIV transmission (AIDSinfo, 2018; Kreitchmann et al., 2012). It is not simply the provision of the ART that is recommended but also the adherence regimen associated with ART. In this case, adherence to ART is defined as patients taking their medication as prescribed, as even minor deviations from this regimen can be detrimental to maternal and neonatal health (Gertsch et al., 2013).

The importance of adherence to ART for HIV has been documented in research literature (Chesney, Ickovics, Hecht, Sikipa, \& Rabkin, 1999; Gertsch et al., 2013; Hecht, Colfax, Swanson, \& Chesney, 1998; Kastrissios, Suárez, Katzenstein, Girard, Sheiner, \& Blaschke, 1998; Kreitchmann et al., 2012). Studies have found that ART adherence lower than $95 \%$ can be associated with the development of viral resistance to medications whereas adherence above $95 \%$ is associated with no opportunistic infection nor deaths (Paterson et al., 2000; Wahl \& Nowak, 2000). Reasons for ART non-adherence include side-effects, being away from home, lack of food and medication, non-disclosure of HIV status to partner, stigma, and work-related demand (Adeniyi et al., 2018). Interestingly, longitudinal studies in Argentina, Brazil, Peru (Kreitchmann et al., 2012) and in Switzerland (Gertsch et al., 2013) found that self-reported adherence among HIV infected pregnant women decreased significantly post-partum.

Similar findings are reported by Nachega et al. (2012) in his meta-analysis of 51 articles of pre- and post-natal HIV infected women from across the world, including seven from South Africa. It was found that overall, that is during and after pregnancy, an estimated $74 \%$ of pregnant women were adequately ( $>80 \%$ ) adherent to their ART regime. Adherence was much higher during pregnancy then after pregnancy (76\% vs 53\% respectively) (Nachega et al., 2012). The decrease in ART adherence during the post-partum period was clarified as the mother's concern regarding the transmission of her HIV to her fetus during pregnancy and birth had abated (Nachega et al., 2012). This is explained by the necessity concerns framework which postulates that a person will adhere to the medication regime if they see a necessity to take the medication and have no concerns about its adverse effects (Clifford, Barber, \& Horne, 2008; Horne et al., 2004). If no necessity is experienced anymore and a concern about adverse effects is seen, medication adherence will decrease. As of 2016, South Africa has the biggest HIV epidemic in the world with 7.1 million people living with HIV, 270,000 new infections annually, 110,000 AIDS related deaths, and the largest ART program in the world with $56 \%$ of adults on ART (UNAIDS, 2017a). In terms of HIV infected pregnant women, 95\% received ART in 2016 and mother- 
to-child transmission (MTCT) of HIV fell from 3.6\% to 1.5\% between 2011 and 2016 (Avert, 2018a). As such, it is possible to eliminate MTCT in South Africa. Peltzer et al. (2017) noted that successful PMTCT interventions in South Africa have included mother-to-mother peer mentoring as well as cognitive behavioral interventions (Futterman et al., 2010). Male involvement in PMTCT was also seen as improving PMTCT outcomes (Ngidi, Naidoo, Ncama, Luvuno, \& Mashamba-Thompson, 2017) as well as interventions that involved numerous text messages and telephone calls to pregnant women reminding them of PMTCT (Geldsetzer et al., 2016). In the 51 study meta-analysis of ART adherence during and after pregnancy, facilitators of better adherence included higher education, higher income, knowledge about PMTCT, previous PMTCT, disclosure of HIV status, positive partner support, support groups, and being on lifelong ART (Nachega et al., 2012). Barriers to ART adherence include but not limited to being younger, drug use, depression, home births, and number of pills (Nachega et al., 2012).

Little is known about longitudinal ART adherence among HIV infected pregnant women in South Africa. The present study aimed to longitudinally examine the impact of a prevention of mother to child transmission (PMTCT) uptake intervention on ART adherence among HIV infected pregnant women in Mpumalanga province, South Africa. It was hypothesised that in the experimental condition mothers would be significantly more likely to adhere to ART protocol medications as prescribed compared to control condition mothers when comparing pre-natal and post-partum adherence rates.

\section{Methods}

\section{Study design}

Study data were drawn from the 'Protect Your Family' (PYF), longitudinal, clinicrandomized, PMTCT controlled trial, conducting a baseline assessment prenatally, and a long-term follow up assessment post-partum (Jones et al., 2014). The baseline assessment was conducted when the women were between 8 and 24 weeks pregnant $(M=18$ weeks, $S D=5.47$ ) while the long-term follow up postpartum assessment was conducted 12 months after birth (see Figure 1). Although the cluster randomized controlled trial (RCT) gathered intermediate measures at 32 weeks pre-natal, 6 weeks and 6 months post-natal, the loss to follow-up was too high to warrant analyses of that data for this paper (Figure 1). The RCT was conducted in 12 community health centres (CHCs) in Gert Sibande and Nkangala districts in Mpumalanga province, South Africa. Randomization was done where HIV infected pregnant women either received a Standard Care (SC) condition or an Enhanced Intervention (EI) condition (Figure 1; Jones et al., 2014). 


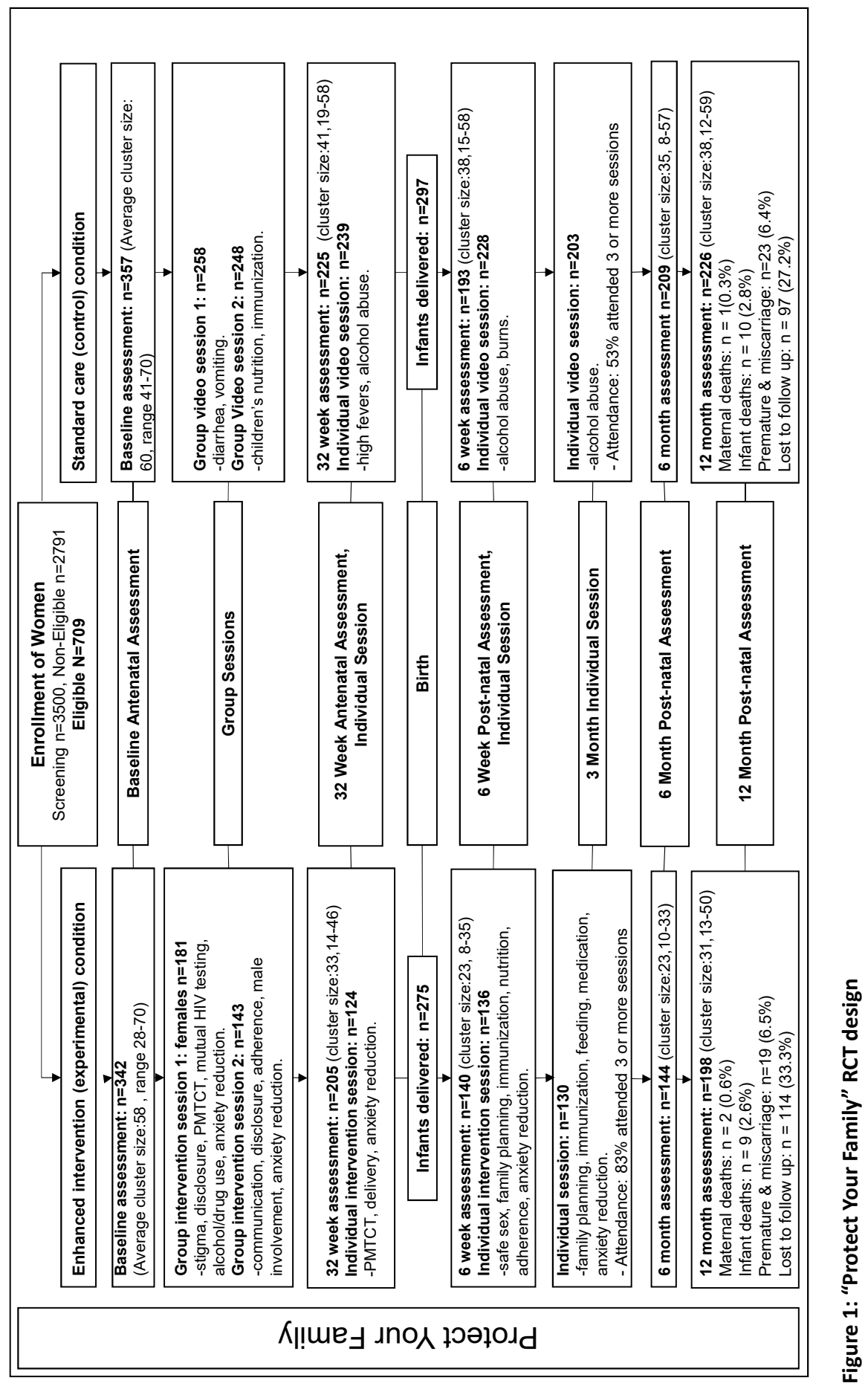




\section{Interventions}

Enhanced intervention (experimental) condition (EI). El participants received the standard PMTCT care offered at all South African CHCs as well as the two group and three individual theory-based social-cognitive PYF intervention (see Figure 1). The standard care consisted of $\mathrm{CHC}$ administered Option B treatment, where the test and treat model was utilized, ensuring that as soon as the pregnant woman was diagnosed as having HIV, she would immediately be enrolled onto ART which would continue after birth only if the mothers health required it (Ramlagan et al., 2018; UNICEF, 2012; WHO, 2012b). The two PYF group intervention sessions of approximately two hours each were held between baseline (8-24 weeks pregnant) and 32 weeks pregnancy (Jones et al., 2014). The groups consisted of between five to seven participants and sessions were conducted in a private room at the $\mathrm{CHC}$ the women were recruited. The facilitators of each session were trained PYF staff member who were lay councillor and were independent of the CHC. Three individual PYF intervention sessions occurred just prior to birth (32 weeks pregnant), as well as 6-weeks and three months post-partum.

The PYF El is a manualized, closed, structured behavioural risk reduction program targeting PMTCT, partner violence, stigma, disclosure, alcohol/drug use, anxiety reduction, communication, disclosure, ART adherence, male involvement, family planning, delivery, safe sex, immunization, nutrition, feeding, and medication (Figure 1; Jones et al., 2014). During the intervention, participants received cognitive behavioural skills training addressing the key components of each session. Participants were encouraged by the PYF staff member to problem solve, provide supportive feedback to each other, and peer mentor one another (Jones et al., 2014). Individual PYF sessions included one-on-one counselling and information sharing sessions where participants were provided with discrete intervention. Thus the intervention as a whole sought to change behaviour as well as influences on behaviour both within the individual and externally by not only providing valuable in-depth information but also by allowing participants to experience the views of others in a similar situation as theirs and gain from the collective. In terms of ART adherence, participants underwent training on what PMTCT is and what it means for them individually and as a group, ART pre- and post-natal, ART for baby, ways to prevent babies from being infected with HIV, the importance of adherence to medication, how to adhere to medication, concerns about ART, confusion and side effects surrounding ART, skipping or missing ART, as well as problems with taking ART including lack of food, transport to get to the $\mathrm{CHC}$, etc. Details regarding the full PYF El can be found in Jones et al., (2014).

Standard care (control) condition (SC). SC participants received the standard PMTCT care (Department of Health, 2013) offered at all South African CHCs as well as health videos on diarrhoea, vomiting, children's nutrition, immunization, high fevers, alcohol abuse and burns (Jones et al., 2014). The standard PMTCT care offered by 
all CHCs included immediate ART initiation should the pregnant women not be on treatment as part of antenatal care, labour and delivery care, as well as postnatal care including baby health, feeding and development (Department of Health, 2013). In addition to the standard PMTCT care offered by $\mathrm{CHC}$ nurses, women enrolled into the $\mathrm{SC}$ condition were shown general health videos utilising a 40 inch flat screen LCD television paired with a DVD player in a private room at the CHC the women were recruited. This ensured that SC participants would spend the same amount of time at the $\mathrm{CHC}$ as the El condition participants. The $\mathrm{SC}$ condition also followed the same protocol timing as the El condition (see Figure 1).

\section{Sample and procedure}

Women were recruited from the 12 sample $\mathrm{CHCs}$ while attending ante-natal care. CHCs were matched according to patient turnover and average antenatal care (ANC) volume which produced 6 matched pairs. In each of the 1:1 matched pair, a computer program was utilized to randomly assign each $\mathrm{CHC}$ as either El or SC (Jones et al., 2014). This cluster randomization allowed for all participants in a specific $\mathrm{CHC}$ to receive the exact same condition and thus cross study contamination could be contained. ANC nurses at each of these $12 \mathrm{CHCs}$, utilizing the indicators of HIV positive and less than six months pregnant, referred potential participants to the PYF staff based at the CHC (Ramlagan et al., 2018). The PYF staff then utilized the study inclusion criteria of being an HIV infected pregnant women, being less than six months pregnant, aged 18 years and older, having a current partner and not been recruited in this study at another $\mathrm{CHC}$ to consecutively sample participants (Jones et al., 2014; Ramlagan et al., 2018). Once screened, eligible women were provided with an informed consent form in a language of their preference including English, isizulu, or seSotho. The information sheet and consent form were read to women who followed along using their own copies. Those women who agreed to enter the survey were required to provide written consent. Recruitment for this RCT began in April 2014 and ended in April 2015. The trial concluded in March 2017 when the last participant completed a 12 month post-partum assessment.

Once consented, women completed a baseline assessment utilizing the Audio Computer-Assisted Self-Interview (ACASI) technology (NIMH Multisite HIV/STD Prevention Trial for African American Couples Group, 2008) loaded onto a touch screen Lenovo ThinkPad X230 tablet/laptop (with $180^{\circ}$ swivel screen) and connected with a Logitech h150 stereo over-ear headphones set (Ramlagan et al., 2018). Trained fieldworkers provided all women with basic training on the usage of the ACASI software as well as the hardware. Training on ACASI was done utilizing the initial demographic variables of the assessment. Once women were comfortable with ACASI, the fieldworkers moved aside to provide confidentiality but stayed in the room should the women have required them. All women who completed a baseline assessment was provided R50.00 (South African Rand = US\$4.72) and R100 for 12 month post-partum assessment. 


\section{Measures}

\section{Antiretroviral adherence}

The main outcome measure of this study was maternal adherence to ART. In South African, during the study time period, pregnant women attending ante-natal clinics were offered free Option B treatment including tenofovir (TDF) + emtricitabine (FTC) or lamivudine (3TC) + efavirenz (EFV) as fixed dose combinations (Department of Health, 2013, 2014). Adherence to these medications were assessed by the Adult AIDS Clinical Trials Group (AACTG) (Chesney et al., 2000) self-reported antiretroviral (ARV) adherence measure. The assessment included a four day recall on the number of ARV medication doses missed that is (a) yesterday, (b) the day before yesterday, (c) three days ago and (d) four days ago. Participants' responses were dichotomized into a score of 0 to indicate that they missed medication in the past four days (nonadherent) or 1 if no medication was missed in the past four days (adherent).

\section{Reasons for missing ART}

The AACTG was utilised to understand the reasons for non-adherence (Chesney et al., 2000). The instrument includes 14 possible reasons for missing ART. Responses to each reason were dichotomized to 1 (Yes) and 0 (No).

\section{Sociodemographic, alcohol, reproductive, HIV, and partner information}

At baseline, personal information about the participants were collected regarding age, educational attainment, relationship status, employment status, alcohol use, income, number of children, if this pregnancy was unplanned, if the women had any HIV infected children, if the women were diagnosed with HIV during this current pregnancy, time in months since ART initiation, if partner is HIV infected, and disclosure of HIV status to partner.

\section{Male involvement}

An 11-item "Male Involvement Index" assessed the women's partner's involvement during the antenatal period (Jones et al., 2014). Questions included "Does your male partner attend antenatal care visits with you," "Have you discussed antenatal HIV prevention for your baby with your male partner," etc. Participants responded to each item as 1 (Yes) or 0 (No), and scores ranged from 0 to 11. Cronbach's $\alpha$ reliability coefficient for the scale at baseline was $\alpha=.83$, and at 12 month postpartum $\alpha=.82$.

\section{Intimate partner violence (IPV)}

The Conflict Tactics Scale 18 (Gelles \& Straus, 1979; Straus, 1979) was used to assess psychological victimization (9-item) and physical violence (9-item) in the past month. The 18 conflict situations presented to women included "discussed the issue calmly," "threw something at you," etc. in the previous month. Responses were recorded on a seven point scale of 0 (never) to 6 (more than 20 times) where a higher score is indicative of increased IPV. Cronbach's Alpha reliability for the 
psychological victimization subscale at baseline was $\alpha=.76$, and at 12 month postpartum $\alpha=.83$. Cronbach's $\alpha$ reliability coefficient for physical violence subscale at baseline was $\alpha=.92$, and at 12 month post-partum $\alpha=.94$.

\section{Stigma}

The nine-item AIDS-Related Stigma Scale (Kalichman et al., 2005) measured externalized stigma as experienced by the women. Items in the scale included "People who have AIDS should be ashamed," "People who have HIV should be isolated," etc. Response options were dichotomous to 0 (disagree) and 1 (agree) resulting in a total scores range of 0 to 9 , where higher scores indicate greater levels of stigma. Due to poor internal reliability $(\alpha=.58)$, the reversed coded item for this scale, question 4, was excluded. Excluding the item, Cronbach's $\alpha$ reliability coefficient for the scale improved to $\alpha=.74$ at baseline, and $\alpha=.69$ at 12 -months.

\section{Depression}

The 10-item "Edinburgh Postnatal Depression Scale 10" (Cox et al., 1987) measured the severity of depression experienced by the women. Women were asked to rate how often they had experienced different symptoms associated with depression during the previous seven days. Questions included "I have been able to laugh and see the funny side of things," "I have felt sad or miserable," etc. Scores ranged from 0 through 30 where the higher the score, the more the likelihood of depression being experienced. Validated cut-off score for South African populations is 12 (Lawrie et al., 1998). Cronbach's $\alpha$ reliability coefficient for the scale at baseline was $\alpha=.66$, and at 12 month post-partum $\alpha=.67$.

\section{Data analysis}

Means, standard deviations, frequencies, and percentages were used to describe the sample. T-tests and Mann-Whiney tests, depending on distributional assumptions, as well as chi-square tests were used to compare adherent and non-adherent participants. Multinomial logistic regression was used by comparing prenatal adherence at baseline with 12 months post-partum adherence. The dependent variable consisted of women reporting nonadherence prenatally and at 12 months follow-up (reference category), women reporting being adherent at baseline and 12-months follow-up, women who began reporting adherence from baseline to 12 month follow-up, and women who stopped reporting being at adherent at 12 months follow-up from baseline. Odds ratios with 95\% confidence intervals were calculated as effect sizes for each of the outcomes (Allen \& Le, 2008). Missing data was handled using multiple imputation with ten imputed datasets(Asparouhov \& Muthén, 2010). The multinomial logistic regression analyses were conducted using Mplus version 7.4 (Muthén \& Muthén, 2014) and all other analyses were conducted using SPSS V24. 


\section{Results}

\section{Attrition analysis}

A total of 683 HIV infected pregnant women completed a baseline assessment and 403 completed a 12 month post-partum assessment. In multivariable logistic regression, education $(A O R=1.68, p=0.014$ ], decreased depression $(A O R=0.69$, $p=0.045$ ], and antiretroviral adherence (AOR $=1.47, p=0.045$ ] were associated with retention in PMTCT care (all $p s<0.05$ ), after controlling for HIV-related stigma and infant HIV status. No other variables were associated with attrition. Variables associated with attrition were included as covariates if they were associated with the outcomes.

\section{Baseline differences between conditions}

Age, education, relationship status, monthly income, number of children, depressive symptoms, and adherence were not different between conditions at baseline. However, the partners of women in the control condition were marginally more likely to be HIV infected ( $28.4 \%$ versus $21.7 \%, p=0.052$ ). Variables that were statistically different between conditions at baseline were included as covariates if they were associated with the outcomes.

\section{Sample characteristics at baseline}

The mean age of the women was 28.40 (SD = 5.71) years (see Table 1 ), with a range of 18 to 46 years. A total of 194 women or $29 \%$ had completed school, $83 \%$ were not employed, $45 \%$ earned less that R600 per month, and 59\% were not married and living separately. Just over half (53\%) of women reported that this current pregnancy was unplanned and a fifth $(20 \%)$ reported that they had no children. Of those that have children, $5 \%$ reported to know that their child is HIV infected. Over half $(54 \%)$ of the women were diagnosed with HIV during this pregnancy with the majority (60\%) having disclosed their HIV status to their partner. The mean time since ART initiation among women was 13.27 (SD = 24.35) months. Over half (54\%) of women reported male involvement during this pregnancy and $13 \%$ reported that they had drunk three or more alcoholic beverages on at least one occasion in the past month.

In Table 1, ART adherence at baseline assessment was positively associated with higher educational attainment, number of children, disclosure of HIV status to partner, and low reported alcohol use. The findings show that physical partner violence, time since ART initiation in months, psychological partner violence, and stigma were negatively associated with adherence to ART. 
Table 1. ART adherence by socioeconomic, reproductive, HIV, partner, alcohol use, stigma and depression at baseline $(N=683)$

\begin{tabular}{|c|c|c|c|c|}
\hline & $\begin{array}{l}\text { All }(N=683) \\
\text { Mean(SD) } \\
n(\%)\end{array}$ & $\begin{array}{c}\text { Nonadherent } \\
\text { (N=224) } \\
\text { Mean(SD) } \\
n(\%)\end{array}$ & $\begin{array}{c}\text { Adherent } \\
\text { ( } \mathrm{N}=457) \\
\text { Mean(SD) } \\
\mathrm{n}(\%)\end{array}$ & $Z / t / x 2, p$ \\
\hline \multicolumn{5}{|l|}{ Sociodemographics } \\
\hline Age $(m, S D)$ & $28.40(5.71)$ & $28.87(5.77)$ & $28.17(5.67)$ & $1.514,0.131$ \\
\hline \multicolumn{5}{|l|}{ Educational attainment } \\
\hline Grade 0-9 & $148(21.7 \%)$ & $67(29.9 \%)$ & $81(17.7 \%)$ & \multirow{3}{*}{$14.998,0.001$} \\
\hline Grade $10-11$ & $339(49.8 \%)$ & $107(47.8 \%)$ & $232(50.8 \%)$ & \\
\hline Grade 12 or more & $194(28.5 \%)$ & $50(22.3 \%)$ & $144(31.5 \%)$ & \\
\hline \multicolumn{5}{|l|}{ Relationship status } \\
\hline Not married, living separate & $403(59.2 \%)$ & $129(57.6 \%)$ & $274(60.0 \%)$ & \multirow{3}{*}{$0.382,0.826$} \\
\hline Not married, living together & $153(22.5 \%)$ & $53(23.7 \%)$ & $100(21.9 \%)$ & \\
\hline Married & $125(18.4 \%)$ & $42(18.8 \%)$ & $83(18.2 \%)$ & \\
\hline \multicolumn{5}{|l|}{ Employment status } \\
\hline Not employed & $562(82.5 \%)$ & $185(82.6 \%)$ & $377(82.5 \%)$ & \multirow{2}{*}{$0.001,0.976$} \\
\hline Employed, Volunteer or Student & $119(17.5 \%)$ & $39(17.4 \%)$ & $80(17.5 \%)$ & \\
\hline \multicolumn{5}{|l|}{ Income (ZAR)per month } \\
\hline$<600$ & $308(45.2 \%)$ & $97(43.3 \%)$ & $211(46.2 \%)$ & \multirow{2}{*}{$0.499,0.480$} \\
\hline$>=600$ or more & $373(54.8 \%)$ & $127(56.7 \%)$ & $246(53.8 \%)$ & \\
\hline \multicolumn{5}{|l|}{ Reproductive } \\
\hline \multicolumn{5}{|l|}{ Number of children } \\
\hline None & $139(20.4 \%)$ & 35 (15.6\%) & $104(22.8 \%)$ & \multirow{2}{*}{$4.707,0.030$} \\
\hline One or more & $542(79.6 \%)$ & $189(84.4 \%)$ & $353(77.2 \%)$ & \\
\hline \multicolumn{5}{|l|}{ Unplanned pregnancy } \\
\hline No & $320(47.0 \%)$ & $95(42.5 \%)$ & $225(49.2 \%)$ & \multirow{2}{*}{$2.810,0.094$} \\
\hline Yes & $361(53.0 \%)$ & $129(57.6 \%)$ & $232(50.8 \%)$ & \\
\hline \multicolumn{5}{|l|}{ HIV issues } \\
\hline \multicolumn{5}{|c|}{ Diagnosed with HIV in this pregnancy } \\
\hline No, before & $314(46.1 \%)$ & $120(53.6 \%)$ & $194(42.5 \%)$ & \multirow{2}{*}{$7.481,0.006$} \\
\hline Yes & $367(53.9 \%)$ & $104(46.4 \%)$ & $263(57.5 \%)$ & \\
\hline Months since ART initiation & $13.27(24.35)$ & $16.41(27.09)$ & $11.73(22.76)$ & $-4.146,<0.001^{*}$ \\
\hline \multicolumn{5}{|l|}{ Partner Issues } \\
\hline \multicolumn{5}{|l|}{ Disclosed HIV status to partner } \\
\hline No & $279(41.0 \%)$ & $71(31.7 \%)$ & $208(45.5 \%)$ & \multirow{2}{*}{$11.867,0.001$} \\
\hline Yes & $402(59.9 \%)$ & $153(68.3 \%)$ & $249(54.5 \%)$ & \\
\hline \multicolumn{5}{|c|}{ Male involvement (cut of $\geq 8$ (median score of Male involvement)) } \\
\hline No & $313(46.0 \%)$ & $102(45.5 \%)$ & $211(46.2 \%)$ & \multirow{2}{*}{$0.024,0.876$} \\
\hline Yes & 368 (54.0\%) & 122 (54.5\%) & 246 (53.8\%) & \\
\hline
\end{tabular}


Table 1. ART adherence by socioeconomic, reproductive, HIV, partner, alcohol use, stigma and depression at baseline $(\mathrm{N}=683)($ cont...)

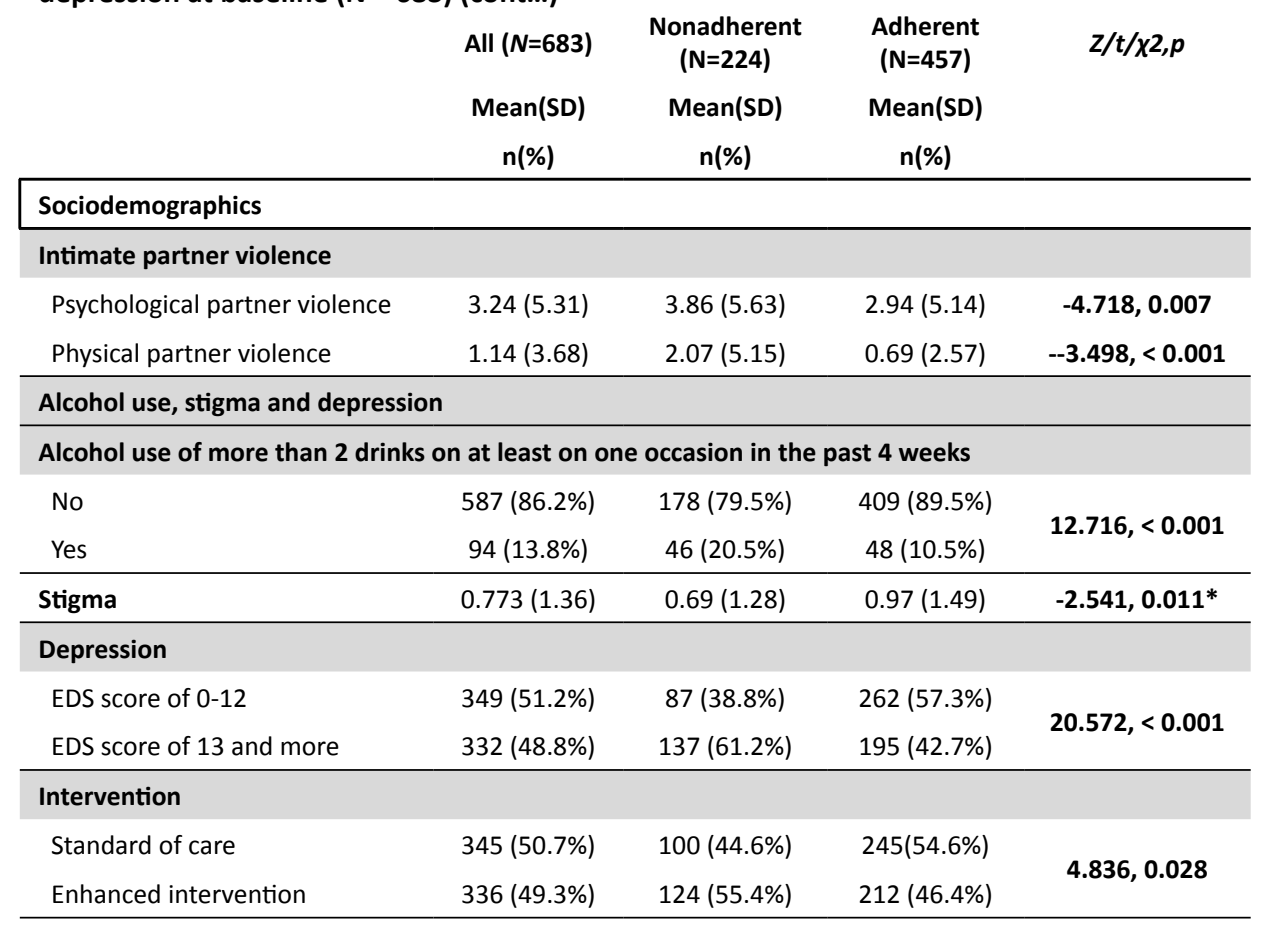

*Mann-Whitney tests were used for median comparison of groups and chi-square tests for differences in proportions.

\section{ART adherence change}

Table 2 below presents the multinomial logistic regression analyses, which shows the odds of women with sustained adherence, changing to adherent and changing to non-adherent from baseline (8-24 weeks prenatal) to 12 months post-partum. In predicting sustained adherence, a significant effect of condition was found suggesting that women in the enhanced intervention condition were less likely to sustain ART adherence over time than women in the standard care condition, after controlling for alcohol use, intimate partner violence, and depressive symptoms. In predicting if women become adherent over time, the intervention condition had no impact on women becoming adherent when controlling for other factors. The intervention condition was significantly associated with change to non-adherence from baseline to 12-month follow-up, which may indicate that the intervention had no long term effect on remaining adherent.

Sustained adherence was also associated with decreased alcohol use (AOR 0.440;

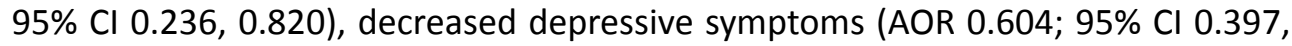
0.21 ), and decreased physical intimate partner violence (AOR 0.886; $95 \% 0.794$, 0.988). Change to adherent was associated with increased depressive symptoms (AOR 2.031; 95\% Cl 1.245, 3.311) and change to non-adherent was not associated with any covariates at $95 \%$ or better. 
Table 2. Multinomial logistic regressions with "Stable non-adherence" (prenatal and 12 months postnatal) as reference group $(n=39)$

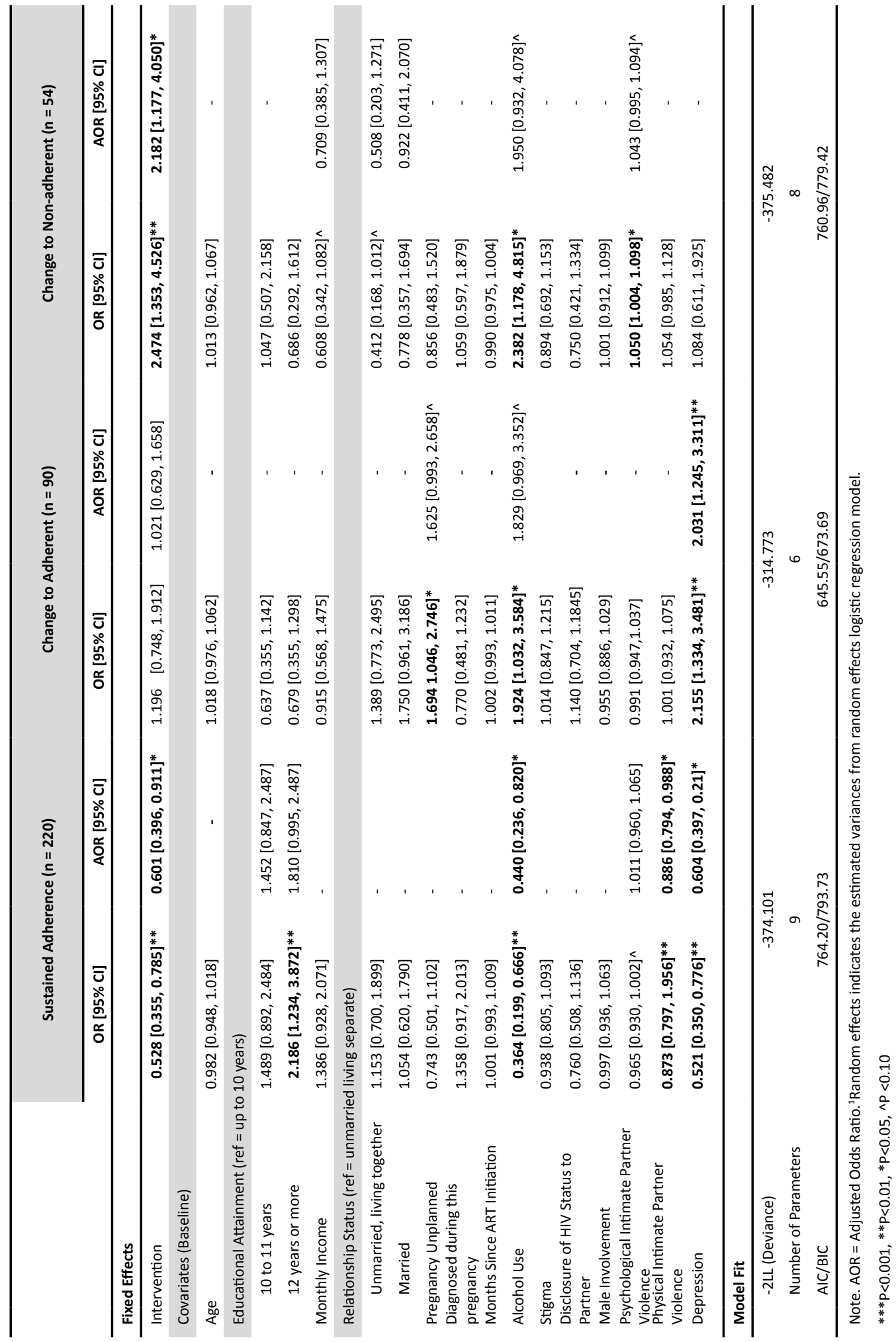




\section{Reason for missing ART}

Table 3 below presents the reasons, by those who were non-adherent, for missing ART by time and condition. Overall, when comparing baseline to 12 month post-partum, the proportion of those giving varying reasons for non-adherence decreased for the majority of reasons. In the control condition, however, increases from baseline to 12 months post-partum were observed for reasons such as "simply forgot", "had too many pills to take", and "felt sick or ill." In the enhanced intervention condition, increases from baseline to 12 months post-partum was observed for reasons such as "had problems taking pills at specified times (with meals, on an empty stomach, etc)", "had problems taking medication due to lack of food" and "ran out of pills."

Table 3: Reason for missing ART by time point and condition

\begin{tabular}{|c|c|c|c|c|}
\hline \multirow[b]{3}{*}{ Reasons } & \multicolumn{2}{|c|}{ Control } & \multicolumn{2}{|c|}{ Enhanced Intervention } \\
\hline & $\begin{array}{l}\text { Baseline } \\
(n=106)\end{array}$ & $\begin{array}{l}12 \text { month } \\
\text { post-partum } \\
(n=45)\end{array}$ & $\begin{array}{l}\text { Baseline } \\
(n=134)\end{array}$ & $\begin{array}{l}12 \text { month } \\
\text { post-partum } \\
(n=69)\end{array}$ \\
\hline & $\%$ & $\%$ & $\%$ & $\%$ \\
\hline Were away from home & 49.1 & 31.1 & 57.5 & 50.7 \\
\hline Were busy with other things & 46.2 & 44.4 & 52.2 & 33.3 \\
\hline Had a change in routine & 37.7 & 35.6 & 38.1 & 31.9 \\
\hline Simply forgot & 34.0 & 42.2 & 44.8 & 30.4 \\
\hline $\begin{array}{l}\text { Did not want others to notice you } \\
\text { taking medication }\end{array}$ & 42.5 & 28.9 & 40.4 & 29.0 \\
\hline $\begin{array}{l}\text { Had problems taking pills at specified } \\
\text { times (with meals, on an empty } \\
\text { stomach, etc.) }\end{array}$ & 39.6 & 20.0 & 34.3 & 36.2 \\
\hline Fell asleep or slept through dose time & 33.0 & 31.1 & 38.1 & 33.3 \\
\hline Felt depressed or overwhelmed & 35.8 & 35.6 & 43.3 & 26.1 \\
\hline Felt like the drug was toxic or harmful & 39.6 & 20.0 & 35.8 & 34.8 \\
\hline Wanted to avoid the side effects & 32.1 & 26.7 & 43.3 & 23.2 \\
\hline Had too many pills to take & 30.2 & 37.8 & 40.3 & 23.2 \\
\hline $\begin{array}{l}\text { Had problems taking medication due } \\
\text { to lack of food }\end{array}$ & 27.4 & 17.8 & 32.8 & 33.3 \\
\hline Felt sick or ill & 26.4 & 33.3 & 30.6 & 23.2 \\
\hline Ran out of pills & 16.0 & 13.3 & 24.6 & 29.0 \\
\hline
\end{tabular}




\section{Discussion}

The study examined the impact, over time, of a multi-session cognitive behavioral PMTCT intervention, including ART adherence, among HIV-infected pregnant women in South Africa. Adherence to antiretroviral medication, which is provided free of charge to all living in South Africa, was defined as taking all ART medication over the last four days. This study found that adherence to ART at baseline was associated with higher educational attainment, number of children, time since ART initiation in months, disclosure of HIV status to partner, both psychological and physical partner violence, the use of alcohol, increased stigma, and increased depression. Similar findings are reported in a meta-analysis among women during and after pregnancy (Nachega et al., 2012).

Between the baseline (8-24 weeks pregnant) assessment and the 12 month postpartum assessment, women in the enhanced intervention group underwent two group and three individual cognitive behavioral PYF intervention sessions. The PYF intervention did not have the desired outcome on remaining adherent nor did it have an impact on becoming adherent over time, which was likely due to the significantly greater proportion of nonadherent women in the experimental condition at baseline (55 versus $46 \%$ ). Because this was a cluster-randomized trial, it is likely that clinics randomized to the experimental condition were more likely to have geographic or area-specific factors that may have negatively impacted adherence, with these factors remaining stable by the 12-month follow-up. The study found that there was a change to nonadherence over time. These results, although distressing for the intervention effect, mimic the findings reported in longitudinal studies among HIV infected women in South America (Kreitchmann et al., 2012), Switzerland (Gertsch et al., 2013), and in the meta-analysis of 51 articles from across the world (Nachega et al., 2012). These studies show that self-reported adherence among HIV infected women decreased significantly post-partum.

The reason for the decrease in ART adherence post-partum was postulated in the meta-analysis which found that the mothers concern surrounding the transfer of HIV to her fetus had abated once the baby was born (Nachega et al., 2012). The necessity-concerns framework (Clifford et al., 2008; Horne et al., 2004) clarifies that the mother adhered to her ART regime as she deemed it necessary to protect the fetus from HIV and thus the concerns for the unborn baby far outweighed the concerns about taking the medication. Once the child was born HIV-negative, the mother had no concern for the transfer of HIV from mother to child and therefore may not have been motivated to continue taking her ART medication. At this juncture, the concerns regarding taking ART far outweighed the necessity for taking it. Once this occurred, post-partum ART adherence decreased. 
The intervention in this study, as seen in Figure 1 above, mainly concentrated on adherence during the pre-natal phase and had only one individual session at 6-weeks post-partum. The lack of adherence reinforcement during the post-partum intervention sessions could have negatively impacted the study outcome. At the 6-week post-partum session, high loss to follow up was experienced due to mothers travelling back to their parents' home for birth. During the study period, the South African government enacted a policy change where Polymerase Chain Reaction (PCR) testing for HIV changed from 6 weeks post-partum to at-birth (Department of Health, 2014). This possibly further led to the high loss to follow up that was experienced at 6 weeks post-partum. The high loss to follow up at 6-weeks meant that women did not receive the adherence intervention post-partum.

Although the ART adherence intervention component of PYF did not have the desired overall outcome of increasing adherence over time as mentioned above, it is important to state that the results did show that for those who sustained their ART adherence over time, this was associated with decreased alcohol use, decreased depressive symptoms, and decreased physical intimate partner violence. These associations were also reported elsewhere in literature (Nachega et al., 2012). In terms of depression, the PYF intervention was effective at reducing depressive symptoms among the study participants (Peltzer et al., 2019) which could have led sustained ART adherence. The PYF intervention also had a desired positive outcome on reducing stigma among study participants (Peltzer et al., 2018) which is a known determinant of non-adherence.

Reasons given by the most number of women in the enhanced intervention group for missing their ART was that they were away from their home when they needed to take their medication. This is similar to a finding in an Eastern Cape study, which stated that being away from home was their second most important reason for missing ART (Adeniyi et al., 2018). In that study population, like this one, when women travelled outside their regular place of treatment, they often travelled without their clinic refill prescription records and thus could not receive ART medication at another clinic. This not only affect those women who travelled in the short term but also those who relocated (Adeniyi et al., 2018) and as such, women in both the Eastern Cape study and this study ran out of pills. The reason of running out of medication in this study was one of three reasons that increased in proportion from baseline to 12 months post-partum.

The remaining two reasons in the enhanced intervention group that increased in proportion from baseline to 12 months post-partum include having problems taking their medication at specified times and, having problems taking their medication due to the lack of food. These reasons were also stated in the Eastern Cape (Adeniyi et al., 2018) where the lack of food lead women, who had sufficient ART, to not take their medication. The lack of food worsens ART side effects (Adeniyi et al., 2018) 
and thus it would lead women not to adhere to their regimen.

\section{Limitations of the study}

The study suffered from high loss-to-follow-up due to the migrant population in Mpumalanga. Although the RCT gathered intermediate measures at 32 weeks pre-natal, 6 weeks and 6 months post-natal, the loss to follow-up was too high to warrant analyses of that data for this paper. The number of participants though at baseline and at 12 months post-partum were sufficient for analysis. The measures utilized were subject to self-report recall bias. Some bias was mitigated by utilizing ACASI. The inclusion criteria biased against women without partners as this study was limited only to women who had a partner. The woman's partner though were not required to be the biological father of the child. Because nonadherence was associated with attrition, it is possible that women who were not adherent may have not been followed up with. Lastly, variables such as knowledge, attitude, norms, skills may have been important to evaluate as these may have influenced adherence.

\section{Conclusion}

The study found that the enhanced intervention had no desired effect on ART adherence over time. It also found that ART adherence decreased post-partum. The high loss to follow up and limited post-partum ART adherence intervention could have led to this outcome. Interventions are thus needed to show the necessity of taking ART post-partum and an increased number of ART adherence interventions are needed during the post-partum phase. Better retention strategies are also necessary at the $\mathrm{CHCs}$ as this study was $\mathrm{CHC}$ based and structured in such a manner as to only interview women when they came for their regular pre- or post-natal $\mathrm{CHC}$ visits. Our high loss-to-follow-up also points to a high loss-to-follow-up of CHC post-partum visits.

Sustained ART adherence was associated with decreased alcohol use, decreased depressive symptoms, and decreased physical intimate partner violence. Due to this finding we recommend that interventions are required to address alcohol use during and after pregnancy, as well as interventions to reduce depression and to increase positive male involvement during and after pregnancy. 


\section{CHAPTER 7}
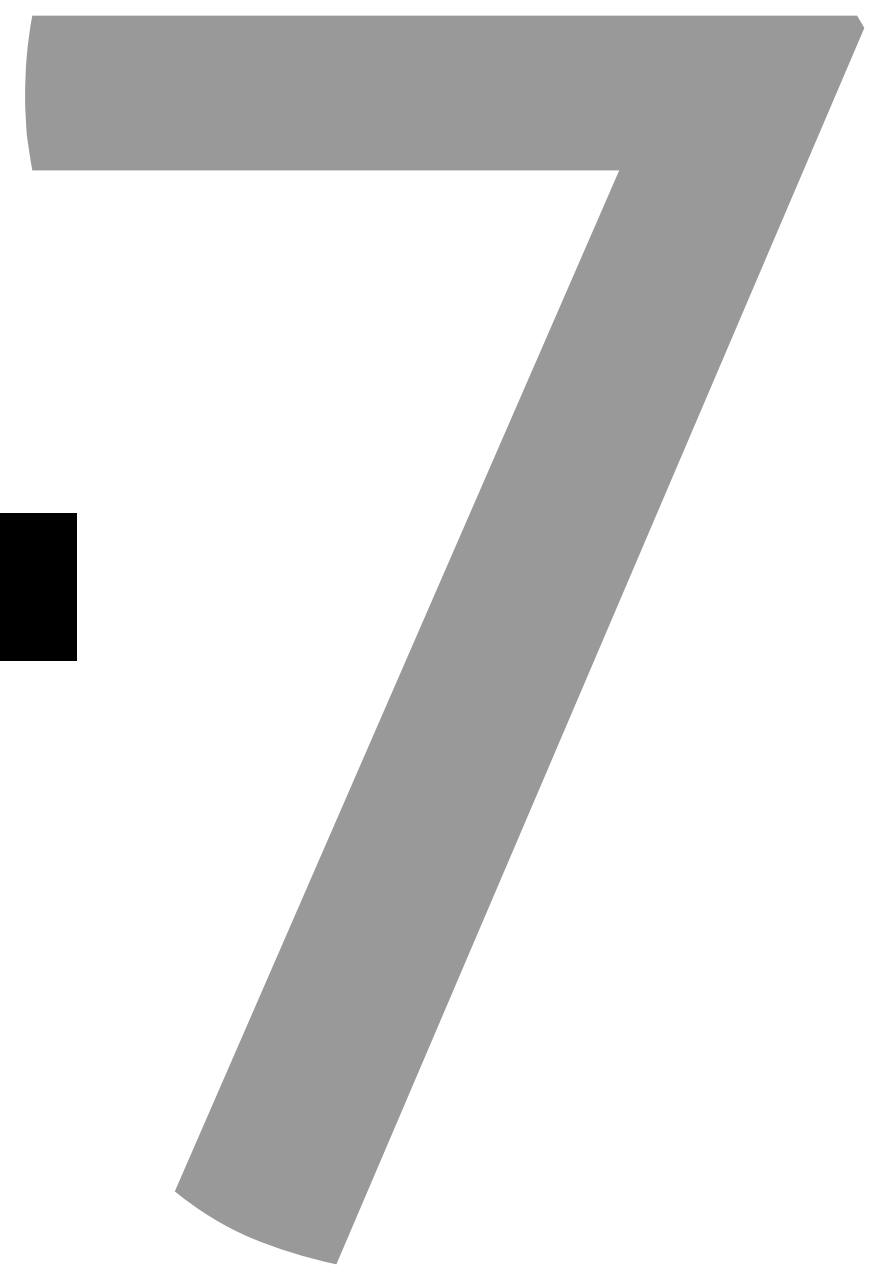
General Discussion 


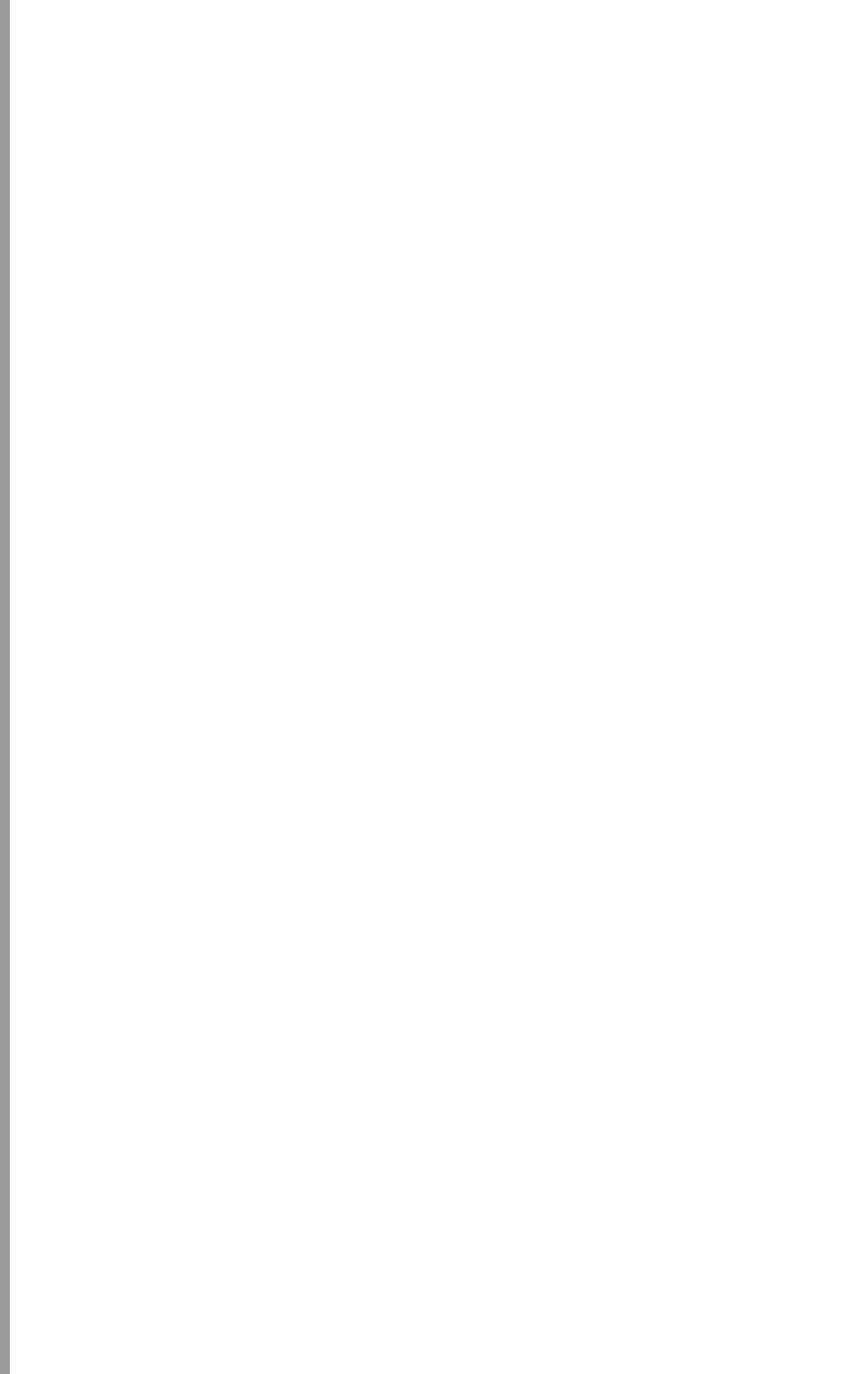




\section{Introduction}

This thesis originated from my very confounding observation, that is, why people, in the case of this thesis, HIV positive pregnant women, do not take their antiretroviral therapy (ART) medication when the medication is readily available and especially when it is given to people who require it, free of charge. In a country which has the highest HIV prevalence in the world and which also has the highest ART rollout in the world, one would expect people living with HIV to take their ART as prescribed. This bewilderment guided me into trying to understand the structural and psychosocial determinants of antiretroviral adherence among pregnant women in Mpumalanga province, South Africa.

In order to understand ART adherence among pregnant women, the first study presented in this thesis explored the prevalence of ART drugs adherence as well as the factors associated with ART drugs adherence among the study population. This study therefore sets the scene in understanding the population group in this thesis. The second study examined the pregnant woman's determinants of HIV-positive status disclosure and non-disclosure. ART adherence was used as a covariate to understand its association with disclosing ones HIV positive status to someone or to one's partner.

The third study explored the HIV positive pregnant women's socioeconomic, behavioral and HIV-related correlates of internalized stigma. In this study, ART adherence was used as a covariate to understand its association with each of the four internalized stigma factors. The fourth study compared the results from two self-reported adherence scales, namely the visual analog scale (VAS) (Giordano et al., 2004) and the Adult AIDS Clinical Trials Group (AACTG) (Chesney et al., 2000) scale, with the respondents dried blood spot (DBS) adherence measure. The final study examined the self-reported change in ART adherence over time and the reasons for missing ART. This final chapter integrates and discusses the results of all five empirical studies in this thesis in order to get a better understanding of ART adherence among pregnant women in Mpumalanga province, South Africa. The chapter also reflects on the strengths and weaknesses of the five empirical studies, and outlines the possible practical implications of the thesis before concluding.

\section{Prevalence and factors associated with ART adherence}

The study population in this thesis consisted of a total of $673 \mathrm{HIV}$-positive pregnant women, with a mean age of 28 years old. Age or more specifically older age was associated with increased adherence to ART as respondents who were of an older age were more likely to be adherent than younger respondents in sample. The old adage that states 'with age comes responsibility' seems to ring true in this population. Of great concern was the high non-adherence among both self- 
reported measures i.e. 11\% VAS non-adherence and 20\% AACTG non-adherence to ART among respondents who were 32 weeks pregnant.

During this 32 week time point, the two self-reported adherence measures were evaluated against dry blood spot (DBS) ART adherence testing in order to compare accuracy of self-report. The data revealed that respondents self-reported higher levels of ART adherence than identified by DBS. When comparing all three measures of adherence, $89 \%$ of respondents' self-reported adherent on the VAS and $80 \%$ self-reported adherent on the AACTG, whereas $74 \%$ were found to be ART adherent according to their DBS result. Previous studies utilizing ART for pre exposure prophylaxis (PrEP) found poor agreement between self-reported adherence measures and biological assessments among women (van Damme et al., 2012). Both research and clinical settings necessitate the use of accurate adherence assessments, and results confirm that adherence by self-report methods should be validated by DBS. However, the use of DBS in routine management of pregnant HIVinfected women is unrealistic due to high costs and delays associated with obtaining results. The results though do indicate that the AACTG four day recall measure for this thesis was the closest to the DBS finding. Interestingly, the AACTG result also showed that some respondents believed that their ART regime consisted of more than one pill a day. This lack of knowledge would compromise adherence since some respondents do not know the number of pills that make up their regime and it is conceivable that they may not know which pill consisted of their ART.

A number of covariates were found to be associated with nonadherence and include alcohol intake, the desire to avoid ART side effects, depression, non-disclosure of ones HIV status, and HIV knowledge. Alcohol is widely accepted as a disinhibitor where increased alcohol intake leads to decreased adherence (Kreitchmann et al., 2012; Mellins et al., 2008; Nachega et al., 2012). Respondents that consumed less than two alcoholic drinks on at least on one occasion in the previous four weeks, were more likely to be adherent to their ART. It would seem that, as the pregnant women consume alcohol, they either forget about their ART, or could not take it due to the lack of privacy, or due to the alcohol/ART interaction.

Respondents who had a desire to avoid the side effects of ART were more likely to not adhere to their prescribed therapy. The side-effects of ART are well documented and include but are not limited to nausea, vomiting, and gastro-intestinal issues (Nachega et al., 2012). When these numerous side-effects are coupled with morning sickness and heartburn during pregnancy (Nachega et al., 2012), it is easy to assume that some pregnant women may not adhere to the ARV treatment regime to ease their discomfort.

Respondents who reported lower depression were more likely to have had better adherence to ART, which supports previous studies that have shown that depression 
is a contributor to ART non-adherence (Mellins et al., 2008; Nachega et al., 2012). It stands to reason that a pregnant woman with little or no depressive symptoms will be more likely to be adherent to her ART when compared to a depressed pregnant woman. It must also be noted that the majority, i.e three quarters, of the study respondents initiated Efavirenz-based ART within the previous 12 months and that just over half were diagnosed HIV-positive during this pregnancy both of which would contribute to their current depression.

Interestingly, all of the above-mentioned contributing factors to ART adherence could be related to the amount of HIV knowledge a respondent may or may not have. The data clearly shows that non-adherence was related to no/low HIV knowledge. With knowledge, i.e. people knowing the advantages of adherence or positive outcome expectations (Bandura, 1986), people are empowered to act to protect themselves and their unborn child (Futterman et al., 2010).

\section{Disclosure and non-disclosure of HIV-positive status}

The lack of disclosure of the pregnant respondents' HIV-positive status was strongly associated with non-adherence of ART (see Chapter 2). In examining disclosure and non-disclosure of HIV positive status more attentively, this thesis found that just over two fifths of respondents did not disclose their HIV positive status to their male partners and over a quarter did not disclose to someone (see Chapter 3). For those who disclosed their HIV status, a strong positive relationship was found between adherence to ART and disclosure. This finding is consistent with previous research in South Africa (Peltzer et al., 2011), Tanzania (Kirsten et al., 2011), and Nigeria (Ekama et al., 2012). Disclosure of ones HIV status was recognized in literature as imperative to combat HIV transmission (Alemayehu et al., 2014; Medley et al., 2004; UNAIDS, 2011; WHO, 2012). Disclosure allows one to receive social support from family and friends, allows for the adoption of safer sexual behaviours, helps prevent mother to child transmission of HIV, and helps make informed reproductive choices (Department of Health, 2014; Shiyoleni, 2013; WHO, 2004)

In trying to understand the reasons for the high prevalence of non-disclosure by the respondents, the thesis investigated the timing of the HIV diagnosis, i.e. diagnosed with HIV during this pregnancy or prior. The finding denotes that over half of the pregnant women found out that they were pregnant during this pregnancy and almost immediately after told that they were HIV positive. This is due to the fact that after the positive result of a pregnancy test is revealed to the now expectant mother, a rapid HIV test is carried out as part of the prevention of mother to child transmission (PMTCT) protocol (Department of Health, 2014). For these newly expectant women who found out that they were both pregnant and HIV positive minutes apart, a statistically significant association was found for non-disclosure of their HIV-positive status. 
This non-disclosure could be explained by the possible grief that may be experienced by the woman testing HIV positive for the first time during pregnancy, as what one could presume is a joyous moment of finding out that you are pregnant is followed almost immediately with the diagnosis of being HIV positive. Even if the woman is shocked by finding out that she is pregnant or did not want to be pregnant, the almost immediate follow up of an HIV positive diagnosis would be devastating. This emotion could be extremely traumatic as the respondents would have found out that they were pregnant and HIV positive almost around the same time they were being enrolled into the study. Literature cites the most common reasons for non-disclosure of HIV status were not being emotionally ready to disclose, fear of negative reactions such as rejection, discrimination, and violence (Visser et al., 2008). However, non-disclosure places pregnant women and their unborn child at greater risk of HIV reinfection, increased viral load, increased levels of depression, and decreases their potential support system emanating from the stigma and discrimination experienced (Shiyoleni, 2013).

\section{Perceived internalised stigma}

Factor analysis was utilized on a 40-item perceived HIV-related stigma scale to identify four HIV-related stigma factors, including personalized stigma, having stigma concerns with public attitudes about people with HIV, having disclosure concerns stigma, and experiencing the stigma of negative self-image (Berger et al., 2001; Lindberg et al., 2014).

This thesis found that women whose household income was classified as low, were more likely to experience three of the four HIV-related stigma factors. Tsai et al. (2015), noted that people from poorest households living with HIV were twice as more at risk of experiencing internalized stigma compared to those living with HIV and from the least poor households. Campbell et al. (2006) notes that poverty is also one of the three main driving forces of maintaining enacted, anticipated and internalised stigma. Respondents that did not complete school, were also more likely to experience personalized stigma. This finding is similar to Sekoni et al. (2012) who found a significant association between higher education and lower levels of personalized stigma as well as the stigma of disclosure concerns. Those with a lower education and lower income may also develop self-deprecating constructions of themselves (Brown et al., 2003; Earnshaw et al., 2013; Rao et al., 2012). This could be as a result of those with lower levels of education also experiencing poorer living and working conditions (Sekoni et al., 2012) as well as they may be less knowledgably about adopting risk-reducing behaviours (Avert, 2018). This burden places HIV positive, stigmatized women at risk of being less able to adopt HIV riskreducing behaviours (Avert, 2018). 


\section{Male partner involvement}

A strong positive relationship between male partner involvement (MPI) during pregnancy and disclosure of HIV status was observed in Chapter 2. Evidence shows that increasing positive male partner involvement in pregnancy can help women and their partners understand and cope with the HIV diagnosis (Albrecht et al., 2006; Maru et al., 2009; Sagay et al., 2006). In addition, women who had an HIV positive partner were more likely to disclose their HIV status to their partners as well as to others. This could imply that the partner could have also disclosed his HIV positive status at some point. Increased ability of women to safely disclose their HIVpositive status to male partners is essential for increased uptake and effectiveness of PMTCT services (Bancheno et al., 2010; Mepham et al., 2011; Tabana et al., 2012). Increased positive MPI has been shown to help provide support for women with HIV diagnosis, which in turn, increase adherence to ART (Albrecht et al., 2006; Maru et al., 2009; Matseke, Ruiter, Rodriguez, Peltzer, Setswe, \& Sifunda, 2017; Peltzer et al., 2011; Sifunda, Peltzer, Rodriguez, Mandell, Lee, Ramlagan, Alcaide, \& Jones, 2019).

Analyses also revealed that having no MPI in the antenatal stage of the pregnancy was significantly associated with experiencing all four of the internalised stigma factors (see Chapter 4). Male involvement in PMTCT has been recognized as a priority area in the elimination of mother-to-child transmission of HIV where their positive involvement in the pregnancy can have an influence in the extended social environment of the family, can increase health seeking behavior, can increase adherence to ART as well as clinic appointments, and assist to mitigate stigma (WHO, 2012). A significant finding leads one to deduce that positive MPI is also critical to assist pregnant women in potentially reducing the stigma they are exposed to.

On the opposite end of positive MPI is intimate partner violence (IPV), which was experienced by a fifth of the study sample (see Chapter 3). Intimate partner violence should not be experienced by anyone. IPV was found to be significantly associated with experiencing personalized stigma, concerns with public attitudes about people with HIV, and experiencing the stigma of negative self-image. Experiencing IPV while HIV positive and pregnant can not only be disastrous to pregnant women, it probably leads to the development of self-deprecating constructions of oneself through the embodiment of shame, guilt, blame and hopelessness (Brown et al., 2003; Earnshaw et al., 2013; Rao et al., 2012). It is this acceptance and subsequent identification with the devalued construction of themselves (Kingori et al., 2012; Rao et al., 2012) that probably leads to increased internalised stigma. This could also be related to AIDS related stigma, which reflects negative beliefs about people living with HIV (Kalichman et al., 2009). AIDS related stigma was experienced by $41 \%$ of the study population, and was only associated in the multivariate logistic regression with the internalized stigma component of negative self-image (see Chapter 4). 


\section{Long-term ART adherence}

A multi-session cognitive behavioral PMTCT intervention was examined. Respondents' baseline (8-24 weeks pregnant) and 12 month post-partum data points were utilized to test an ART adherence intervention and understand ART adherence change over time (see consort diagram in Chapter 6, Figure 1). The intervention did not have the desired outcome on respondents in the experimental condition remaining adherent over time. The intervention also did not have an impact on respondents in the experimental condition becoming adherent over time. There are a few possible reasons for these intervention effect findings.

A potential reason for intervention failure could stem from the study design of being a cluster-randomized trial. It is probable that clinics randomized to the experimental condition were more likely to have area-specific factors that may have negatively impacted on adherence, with these factors remaining stable by the 12-month follow-up and overruling the impact of a temporary intervention. Randomization was done at clinic level where the entire clinic was randomized into either experimental or control condition. In this way, it was believed that participants in the control condition would not be influenced by participants in the experimental condition and vice versa. It must also be noted that there was significantly greater proportion of non-adherent women at baseline in the experimental condition than in the control condition ( $55 \%$ versus $46 \%$ ).

It was also found that there was a change to nonadherence over time and although a distressing intervention effect, the finding mimics reported longitudinal studies among HIV infected women from around the world (Gertsch et al., 2013; Kreitchmann et al., 2012; Nachega et al., 2012). These studies show that self-reported adherence among HIV infected women decreased significantly post-partum and could be attributed to a decline in the mothers concerns surrounding the transfer of HIV to their fetus after birth (Nachega et al., 2012). The necessity-concerns framework is a theory that states that a person will adhere to their medication regime if they see the necessity to take the medication and where their concerns about the medication and its side-effects are low (Clifford et al., 2008; Horne et al., 2004). Should the concerns about the medication ever outweigh the necessity to take it, then people will stop taking their medication (Horne et al., 2004). In terms of this thesis, the balancing act of the necessity-concerns framework clarifies that the pregnant woman adhered to her ART regime as she deemed it necessary to protect her fetus from HIV and thus the concerns for the unborn baby outweighed the concerns about taking the medication. Once the child was born HIV-negative, the mother had no concern for the transfer of HIV from mother to child and therefore may not have seen the necessity to continue taking her ART, and thus, as the concerns about taking the medication grew, post-partum ART adherence decreased. At this juncture, the concern about taking ART outweighed the necessity to take it. 
The lack of adherence reinforcement during the post-partum intervention sessions could have also negatively impacted this outcome. In terms of the way the ART adherence intervention was scheduled, the majority of the ART intervention sessions were carried out during the ante-natal phase and there was minimal adherence intervention during the post-natal phase (see consort diagram in Chapter 6, Figure 1). During the post-natal phase, high loss to follow-up was experienced. Both the high loss to follow up and limited ART adherence intervention during the post-natal phase of the study resulted in a lack of intervention for the majority of respondents. Respondents who were non-adherent were asked the reasons for missing their ART at baseline and at 12 month post-partum. The number one reason given by the majority of women in the enhanced intervention group for missing their ART was that they were away from their home when they needed to take their medication. The reason of running out of medication in this study was one of three reasons that increased in proportion from baseline to 12 months post-partum. The remaining two reasons in the enhanced intervention group that increased in proportion from baseline to 12 months post-partum include having problems taking their medication at specified times and, having problems taking their medication due to the lack of food. These reasons are similar to an Eastern Cape study, which found that when women travelled outside their regular place of treatment, they often travelled without their clinic refill prescription records and thus could not receive ART at another clinic (Adeniy et al., 2018). This not only affected those women who travelled in the short term but also those who relocated (Adeniy et al., 2018) and as such, women in both an Eastern Cape study and this study ran out of pills.

\section{Strengths and weaknesses}

This thesis specifically investigated HIV positive women on Option B treatment. With Option B, women who are pregnant and HIV positive would be immediately initiated on ART, but the ART treatment will only continue after the cessation of breastfeeding if the mother's health requires it (Besada et al., 2017). There is currently limited research on Option B treatment regime for pregnant women, as well as limited research on ART adherence in general among pregnant women (Nachenga et al., 2012). This thesis was therefore not able to compare its findings to other similar research on Option B and thus had to rely on other ART regimes for comparison.

It is evident from the AACTG data that respondents believed that their ART regime consists of more than one pill per day. As such, the AACTG measure was calculated using all medication the respondents believe consists of their ART regime. As an immediate intervention, an education program should be put in place, to educate all people, whether HIV positive or not, on the current ART regime available in South Africa. This will not only help those who are currently on ART, but also those who may be afraid of going onto the regime and all those who support people on 
ART regime. It is also plausible that the respondents did not know which of their medication made up their ART and thus the self-reported adherence was greater when compared to the ART DBS test results. It this way, it is important for both clinicians and researchers to ensure that patients and respondents first understand what their ART regime consists of before they self-report their adherence.

A cross-sectional study design was used for four of the five empirical studies in this thesis. In this type of design, although good for planning, monitoring and evaluation, it is essentially a single time-point measure to determining the association between an outcome and risk factors of the study respondents'. This type of design therefore cannot infer causality. The design also led to analysis where many of the same variables were used as either the primary outcome measure or the explanatory measures. The final empirical study in this thesis, rectifies some of this by utilizing a longitudinal, clinic-randomized, PMTCT controlled trial design where the effects of an adherence intervention were tested.

Only self-report measures were used in the majority of this thesis, which predisposes respondents to social desirable answering and may introduce a recall bias. Some bias was mitigated by utilizing ACASI. Nevertheless, the findings of this thesis add to existing literature. In terms of comparing DBS and self-report adherence measures, this was only done at one time point, 32 weeks pregnant, due to the cost of DBS testing. Preceding the 32 week time point was a baseline questionnaire and two group intervention sessions (see consort diagram in Chapter 6, Figure 1).

Finally, the inclusion criteria of this study biased against women without partners as only women who had a partner were included. The woman's partner was not required to be the biological father of the child nor was he ever interviewed.

\section{Practical implications}

In order to achieve a PMTCT goal of first reducing and then eliminating HIV transmission from mother to child, thereby ensuring all babies born are HIVnegative and remain that way for their first year of their lives, adherence to ART is a necessary step. However, encouraging people to take their ART is complicated and requires a number of simultaneous interventions. Thus, there are a number of suggestions stemming from this thesis, as well as potential future studies that are required to better understand the situation of ART adherence among HIV-positive pregnant women living in Mpumalanga province, South Africa.

The final outcome of the thesis found that respondents took their ART ante-natally but stopped taking their medication once their baby was born and they realized that their baby was no longer in danger of contracting HIV. Following the necessityconcerns framework (Horne et al., 2004) and utilising an Intervention Mapping 
framework (Bartholomew Eldredge, Markham, Ruiter, Fernandez, Kok, \& Parcel, 2016), interventions should be developed to show the necessity and mitigate the concern of adhering to the ART regime post-partum. Logic models should be used to better understanding of the problems, guide the development of interventions and continuously evaluate the interventions, making changes as and when necessary (Bartholomew Eldredge et al., 2016).

The high loss to follow-up, and thus lack of intervention, could have led to poorer ART adherence. Better retention is thus necessary at the clinics as this study was clinic based and designed in such a manner as to only interview and provide interventions to women when they came for their regular clinic visits for child vaccinations. The high loss-to-follow-up in the study inadvertently points to a high loss-to-follow-up of women at clinic level during post-partum visits and thus needs to be improved. It was expected that women would bring their child to the clinics for their vaccinations but it would seem that the mothers themselves either did not bring their child in for the vaccination or went elsewhere for the vaccination. This high loss to follow-up could be mitigated by increasing the amount of community outreach done by each clinic. Currently, there are clinic outreach available but this outreach need to be extended to pregnant women and new mothers and should include both medical and psychosocial care. Another possible solution is to create a nationally linked electronic patient file network where all patients can access health care in any public clinic/hospital in South Africa and have their health files readily available to them.

Disclosure of ones HIV positive status came up numerous times during the course of this thesis. It was seen that disclosing ones status is protective, can lead to ART adherence, and thus it is suggested that programs that encourage disclosure of one's HIV and pregnancy status are needed and need to be strengthened. The findings of this study highlight the need to encourage disclosure as a way to promote ART adherence among HIV positive pregnant women. The findings also highlight the importance of male partner involvement during pregnancy in order to encourage disclosure. This thesis recommends increased psychosocial support for newly diagnosed HIV positive pregnant women in order to encourage disclosure to their male partners. Disclosure is also related to stigma where those who feel stigmatized are more likely not to disclose, which in turn negatively affects adherence to ART. An all-encompassing program that deals with disclosure of HIV positive status, positive male involvements as well as stigma and IPV reduction is needed.

Consequence theory (Serovich, 2001) could be utilized to understand the problem and design an intervention. In consequence theory, disclosure is more likely to occur once the reward for disclosing outweighs the cost of disclosing. As seen above, this thesis found that those that did disclose their HIV status had a partner who was also HIV positive. In these cases, it would seem that the reward for disclosing was 
higher than the cost. Intervention programs would need to be developed to exploit this finding.

It has also been shown that male partners need to be encouraged to be positively involved in their partner's pregnancy. The male partners need to form part of PMTCT programs or at minimum they need to form part of ante-natal care as it is protective. Positive male involvement leads to better social support for the women and better adherence to ART. On the opposite end of the scale of positive male involvement is IPV. IPV has been shown to increase HIV-related stigma among study sample and the lack of positive male support would inadvertently lead to a drop in $A R V$ adherence. Coupled with increased positive male involvement programs, is the need for initiation of IPV prevention programs. Support groups for both women and men need to be set up to mitigate IPV as well as to cope should it happen.

Respondents in this study who reported lower rates of depression had better adherence to ART and studies have shown that depression is a contributor to ART non-adherence. Support groups for women would provide them with a safe space to talk through their issues, gain vital information and assist them with their depression. Literature has well documented the success of social support and support groups for HIV positive people who are also exhibiting depressive symptoms (Hays, Turner, \& Coates, 1992; Jalali, Hasani, Hashemi, Kimiaei, \& Babaei, 2019; Mao, Qiao, Li, Zhao, Zhou, \& Shen, 2019; Simoni, Pantalone, Plummer, \& Huang, 2007). Not only does social support and support groups help in some cases maintain depression and even help with reducing depression among HIV positive people, it also assists with increasing adherence to ART, and reducing stigma (Hays et al., 1992; Jalali et al., 2019; Mao et al., 2019; Simone et al., 2007). Programs designed to help mothers with antenatal and postnatal depression as well as HIV depression need to be investigated and encouraged. Programs could be designed with the aid of Intervention Mapping and Bandura's social cognitive theory which states people are shaped by behavior, thought and their environment (Bartholomew Eldredge et al., 2016). By observing others in support groups and by talking with people who are in a similar situation, it is hoped that those who are depressed are better able to understand their situation and thus better deal with it. It is important to note that Intervention Mapping (Bartholomew Eldredge et al., 2016) should be utilized to identify, modify or create new interventions that will holistically tackle ART adherence. Depression could be caused or magnified by the side effects of ART and as such, it is also advised that closer monitoring of ART side effects by clinicians is required to mitigate its effects and increase adherence.

The finding that an extremely high $72 \%$ of women aged 18 years and older in Mpumalanga have not completed schooling is shocking and needs to the tackled immediately. The results obtained in this thesis reveal the ways in which higher levels of education, income and partner involvement (outside of the context of IPV) 
serve as protective factors against internalized stigma, at multiple layers. In order to tackle improved schooling outcomes, increased funding should be made available for adult literacy programs and should be coupled with targeted advertising that promotes the necessity and availability of adult literacy. The current public schools together with its educators could easily be used as a venue and resource for such activities during after school and evening time periods to accommodate the adults. In order to make the resources of funding, venue and educators available, current education policies need to be amended. Scholars who are still in the schooling system should also be targeted to ensure that they complete their schooling. These potential interventions are outside the scope of this thesis and as such, should be used as a starting point for a study utilising Intervention Mapping (Bartholomew Eldredge et al., 2016).

The thesis also tested the Berger et al. (2001) 40-item perceived internalized stigma scale which was shown to be a valid and reliable scale. Like the original factor analysis carried out on this scale, a four factor solution of internalised stigma was realised in a South African context. The scale will benefit from more evaluation among different sub-population groups including among all people living in rural areas as well as among vulnerable populations in South Africa. In this thesis, the full 40-item perceived internalized scale was utilised only among a sample of HIV positive pregnant women in Mpumalanga province and as such more evaluation among different sub-populations and within different areas of South Africa will allow validity and reliability testing of the scale in different contexts, thus strengthening motivation to utilise the scale.

Finally, the study found that most adherence assessments rely on self-report and are subject to recall and social desirability bias, as well as patients/respondents not understanding what constitutes their ART regime. As such, physicians and researchers may overestimate adherence. Due to this, strategies are needed to enhance and accurately evaluate adherence to ART among this vulnerable population. As mentioned above, physicians/researchers should explain to patients/ respondents what constitutes their ART regime before allowing them to self-report. Although ART adherence testing through DBS is expensive, it should be considered in cases when physicians deem the self-report to be ineffective.

\section{Conclusion}

Adhering to the ART protocol is not simply a matter of providing free ART medication to all those who require it and expecting them to then take their medication as prescribed. Adherence to ART is multifaceted and complicated. There are numerous influences that affect adherence including alcohol usage, avoidance of ART sideeffects, knowledge, male partner involvement, intimate partner violence, stigma, depression, disclosure of status, and loss to follow-up to name a few. The thesis 
also showed that adherence to ART changes with time and belief. People will take their ART if they believe it's necessary but will not take their medication if they have a concern about the medication. As such, by utilising the necessity-concerns framework (Horne et al., 2004, 2013) , if the necessity to take ART is conveyed to people and then maintained, while at the same time the concerns about ART are mitigated, then it stands to reason that adherence to ART will increase. If one wants to increase adherence, each of the above mentioned determinants cannot be dealt with individually as they are interconnected and require a holistic approach.

\section{Final thought}

So at the end of this thesis, one might ask, 'Have You' walked a mile in their shoes, and the answer is no, not even close. There is still so much more to understand.... 

REFERENCES 


\section{REFERENCES}

Adeniyi, O. V., Ajayi, A. I., Goon, D. Ter, Owolabi, E. O., Eboh, A., \& Lambert, J. (2018). Factors affecting adherence to antiretroviral therapy among pregnant women in the Eastern Cape, South Africa. BMC Infectious Diseases, 18, 175.

AIDSinfo. (2018). Guidelines for the Use of Antiretroviral Agents in Adults and Adolescents Living with HIV. In Department of Health and Human Services. Retrieved from https:// aidsinfo.nih.gov/contentfiles/lvguidelines/adultandadolescentgl.pdf

Albrecht, S., Semrau, K., Kasonde, P., Sinkala, M., Kankasa, C., Vwalika, C., ... Kuhn, L. (2006). Predictors of Nonadherence to Single-Dose Nevirapine Therapy for the Prevention of Mother-to-Child HIV Transmission. JAIDS Journal of Acquired Immune Deficiency Syndromes, 41(1), 114-118. https://doi.org/10.1097/01.qai.0000179425.27036.d7

Alemayehu, M., Yohannes, G., Damte, A., Fantahun, A., Gebrekirstos, K., Tsegay, R., Goldberger, A., Yebyo, H. (2015). Prevalence and predictors of sexual violence among commercial sex workers in Northern Ethiopia. Reproductive Health, 12(1), 47. https:// doi.org/10.1186/s12978-015-0036-5

Allen, J., \& Le, H. (2008). An additional measure of overall effect size for logistic regression models. Journal of Educational and Behavioral Statistics, 33(4), 416-441. https://doi. org/10.3102/1076998607306081

Alonzo, A. A., \& Reynolds, N. R. (1995). Stigma, HIV and AIDS: An exploration and elaboration of a stigma trajectory. Social Science \& Medicine, 41(3), 303-315. https://doi. org/10.1016/0277-9536(94)00384-6

Asparouhov, T., \& Muthén, B. (2010). Multiple Imputation with Mplus. Retrieved from https://www.statmodel.com/download/Imputations7.pdf

Avert. (2018a). HIV and AIDS in South Africa. Retrieved May 4, 2018, from Avert website: https://www.avert.org/professionals/hiv-around-world/sub-saharan-africa/southafrica\#footnote71_zwiym3r

Avert. (2018b). Women and girls, HIV and AIDS. Retrieved March 4, 2018, from Avert website: https://www.avert.org/professionals/hiv-social-issues/key-affected-populations/ women

Avert. (2019). HIV drug resistance. Retrieved May 20, 2019, from https://www.avert.org/ node/2716/pdf

Bajunirwe, F., \& Muzoora, M. (2005). Barriers to the implementation of programs for the prevention of mother-to-child transmission of HIV: a cross-sectional survey in rural and urban Uganda. AIDS Research and Therapy, 2(1), 10. https://doi.org/10.1186/17426405-2-10

Bancheno, W. M., Mwanyumba, F., \& Mareverwa, J. (2010). Outcomes and challenges of scaling up comprehensive PMTCT services in rural Swaziland, Southern Africa. AIDS Care, 22(9), 1130-1135. https://doi.org/10.1080/09540121003615079

Bandura, A. (1986). Social Foundations of Thought and Action: A Social Cognitive Theory. Englewood Cliffs, NJ: Prentice-Hall.

Bangsberg, D., Hecht, F., Charlebois, E., Chesney, M., \& Moss, A. (2001). Comparing objective measures of adherence to HIV antiretroviral therapy: electronic 
medication monitors and unannounced pill counts. AIDS and Behavior., 5(3), 275-281. Retrieved from https://idp.springer.com/authorize/casa?redirect uri=https://link.springer.com/article/10.1023/A:1011396711486\&casa_ token=skjI0CCUaVwAAAAA:QkckmCARz07qXBcCFrI6cRruye5la_Lb4UOWMogoaZKp4NKs384lzSnn2-4OazhkfULgdp40RAWVtWo

Bartholomew Eldredge, L.K., Markham, C.M., Ruiter, R.A.C., Fernandez, M.E., Kok, G., \& Parcel, G.S. (2016). Planning health promotion programs: An intervention mapping approach ( $4^{\text {th }}$ ed.). San Francisco: Jossey-Bass.

Bateman, C. (2013). Much ado over the new South African PMTCT guidelines. South African Medical Journal, 103(4), 218. https://doi.org/10.7196/samj.6880

Bell, E., Mthembu, P., O'Sullivan, S., \& Moody, K. (2007). Sexual and reproductive health services and HIV testing: perspectives and experiences of women and men living with HIV and AIDS. Reproductive Health Matters, 15(29 SUPPL.), 113-135. https://doi. org/10.1016/S0968-8080(07)29029-9

Berger, B. E., Ferrans, C. E., \& Lashley, R. (2001). Measuring stigma in people with HIV : psychometric assessment of the HIV stigma scale. Research in Nursing \& Health, 24, 518-529.

Berwick, D. (2002). "We all have AIDS": case for reducing the cost of HIV drugs to zero. BMJ, 324(7331), 214-218. https://doi.org/10.1136/bmj.324.7331.214

Besada, D., Van Cutsem, G., Goemaere, E., Ford, N., Bygrave, H., Lynch, S. (2017). The case for Option B and Optional B+: Ensuring that South Africa's commitment to eliminating mother-to-child transmission of HIV becomes a reality. Southern African Journal of HIV Medicine, 13(4), 178. https://doi.org/10.4102/sajhivmed.v13i4.112

Bos, A. E. R., Pryor, J. B., Reeder, G. D., \& Stutterheim, S. E. (2013). Stigma: Advances in Theory and Research. Basic and Applied Social Psychology, 35(1), 1-9. https://doi.org /10.1080/01973533.2012.746147

Bos, A. E. R., Schaalma, H. P., \& Pryor, J. B. (2008). Reducing AIDS-related stigma in developing countries: The importance of theory- and evidence-based interventions. Psychology, Health and Medicine, 13(4), 450-460. https://doi.org/10.1080/13548500701687171

Brou, H., Djohan, G., Becquet, R., Allou, G., Ekouevi, D. K., Viho, I., ... Desgrées-du-Loû, A. (2007). When do HIV-infected women disclose their HIV status to their male partner and why? A study in a PMTCT programme, Abidjan. PLoS Medicine, 4(12), e342. https://doi.org/10.1371/journal.pmed.0040342

Brown, L., Macintyre, K., \& Trujillo, L. (2003). Interventions to reduce HIV/AIDS stigma: What have we learned? AIDS Education and Prevention, 15(1), 49-69. https://doi. org/10.1521/aeap.15.1.49.23844

Byamugisha, R., Tumwine, J. K., Semiyaga, N., \& Tylleskär, T. (2010). Determinants of male involvement in the prevention of mother-to-child transmission of HIV programme in Eastern Uganda: a cross-sectional survey. Reproductive Health, 7(1), 12. https://doi. org/10.1186/1742-4755-7-12

Campbell, C., Nair, Y., \& Maimane, S. (2006). AIDS stigma, sexual moralities and the policing of women and youth in South Africa. Feminist Review, 83(1), 132-138. https://doi. org/10.1057/palgrave.fr.9400285

Carey, M. P., \& Schroder, K. E. E. (2002). Development and psychometric evaluation of the 
brief HIV Knowledge Questionnaire. AIDS Education and Prevention, 14(2), 172-182. https://doi.org/10.1521/aeap.14.2.172.23902

Carr, R. L., \& Gramling, L. F. (2004). Stigma: A health barrier for women with HIV/ AIDS. Journal of the Association of Nurses in AIDS Care, 15(5), 30-39. https://doi. org/10.1177/1055329003261981

Castillo-Mancilla, J.R., Searls, K., Caraway, P., Zheng, J-H., Gardner, E.M., Predhomme, J., Bushman, L.R., Anderson, P.L., Meditz, A. L. (2014). Short Communication: Tenofovir Diphosphate in dried blood spots as an objective measure of adherence in HIVinfected women. AIDS Research and Human Retroviruses, 31(4), 428-432. https://doi. org/10.1089/aid.2014.0229

Castro, A., \& Farmer, P. (2005). Understanding and addressing AIDS-related stigma: From anthropological theory to clinical practice in Haiti. American Journal of Public Health, 95(1), 53-59. https://doi.org/10.2105/AJPH.2003.028563

Catz, S. L., Kelly, J. A., Bogart, L. M., Benotsch, E. G., \& McAuliffe, T. L. (2000). Patterns, correlates, and barriers to medication adherence among persons prescribed new treatments for HIV disease. Health Psychology, 19(2), 124-133. Retrieved from http:// www.ncbi.nlm.nih.gov/pubmed/10762096

Chesney, M.A., Ickovics, J.R., Chambers, D.B., Gifford, A.L., Neidig, J., Zwickl, B. (2000). Selfreported adherence to antiretroviral medications among participants in HIV clinical trials: The AACTG Adherence Instruments. AIDS Care, 12(3), 255-266. https://doi. org/10.1080/09540120050042891

Chesney, M. A., Ickovics, J., Hecht, F. M., Sikipa, G., \& Rabkin, J. (1999). Adherence: a necessity for successful HIV combination therapy. AIDS, 13(Suppl A), S271-8. Retrieved from https://europepmc.org/abstract/med/10885784

Chesney, M. A., \& Smith, A. W. (1999). Critical Delays in HIV Testing and Care. American Behavioral Scientist, 42(7), 1162-1174. https://doi.org/10.1177/00027649921954822

Cingolani, A., Antinori, A., Rizzo, M.G., Murri, R., Ammassari, A., Baldini, F., Di Giambenedetto, S., Cauda, R., De Luca, A. (2002). Usefulness of monitoring HIV drug resistance and adherence in individuals failing highly active antiretroviral therapy: A randomized study (Argenta). AIDS, 16(3), 369-379. https://doi.org/10.1097/00002030-20020215000008

Clifford, S., Barber, N., \& Horne, R. (2008). Understanding different beliefs held by adherers, unintentional nonadherers, and intentional nonadherers: Application of the NecessityConcerns Framework. Journal of Psychosomatic Research, 64(1), 41-46. https://doi. org/10.1016/j.jpsychores.2007.05.004

Colombini, M., James, C., Ndwiga, C., \& Mayhew, S. H. (2016). The risks of partner violence following HIV status disclosure, and health service responses: narratives of women attending reproductive health services in Kenya. Journal of the International AIDS Society, 19(1), 20766. https://doi.org/10.7448/IAS.19.1.20766

Cox, J. L., Holden, J. M., \& Sagovsky, R. (1987). Detection of postnatal depression. Development of the 10-item Edinburgh Postnatal Depression Scale. The British Journal of Psychiatry: The Journal of Mental Science, 150, 782-786. Retrieved from http:// www.ncbi.nlm.nih.gov/pubmed/3651732

Crandall, C. S., \& Moriarty, D. (1995). Physical illness stigma and social rejection. British 
Journal of Social Psychology, 34(1), 67-83. https://doi.org/10.1111/j.2044-8309.1995. tb01049.x

Crawford, A. M. (1996). Stigma Associated With AIDS: A Meta-Analysis. Journal of Applied Social Psychology, 26(5), 398-416. https://doi.org/10.1111/j.1559-1816.1996. tb01856.x

Davis, D. C. (1996). The Discomforts of Pregnancy. Journal of Obstetric, Gynecologic \& Neonatal Nursing, 25(1), 73-81. https://doi.org/10.1111/j.1552-6909.1996.tb02516.x

Deeks, S. G., Lewin, S. R., \& Havlir, D. V. (2013). The end of AIDS: HIV infection as a chronic disease. The Lancet, Vol. 382, pp. 1525-1533. https://doi.org/10.1016/S01406736(13)61809-7

Department of Health. (2012). The 2011 National Antenatal Sentinel HIV \&amp; Syphilis Prevalence Survey in South Africa. Pretoria: Department of Health.

Department of Health. (2013). The South African Antiretroviral Treatment Guidelines, 2013. Pretoria: Department of Health.

Department of Health. (2014). National consolidated guidelines for the prevention of mother-to-child transmission of HIV (PMTCT) and the management of HIV in children, adolescents and adults. In Department of Health. Retrieved from https:// www.bps.go.id/dynamictable/2018/05/18/1337/persentase-panjang-jalan-tol-yangberoperasi-menurut-operatornya-2014.html

Desgrées-du-Loû, A., Brou, H., Traore, A. T., Djohan, G., Becquet, R., \& Leroy, V. (2009). From prenatal HIV testing of the mother to prevention of sexual HIV transmission within the couple. Social Science \& Medicine, 69(6), 892-899. https://doi.org/10.1016/j. socscimed.2009.05.045

Earnshaw, V. A., \& Chaudoir, S. R. (2009). From conceptualizing to measuring HIV stigma: A eview of HIV stigma mechanism measures. AIDS and Behavior, 13(6), 1160-1177. https://doi.org/10.1007/s10461-009-9593-3

Earnshaw, V. A., Smith, L. R., Chaudoir, S. R., Amico, K. R., \& Copenhaver, M. M. (2013). HIV stigma mechanisms and well-being among PLWH: A test of the HIV stigma framework. AIDS and Behavior, 17(5), 1785-1795. https://doi.org/10.1007/s10461-013-0437-9

Earnshaw, V., Bogart, L., Dovidio, J., \& Williams, D. (2015). Stigma and racial/ethnic HIV disparities: moving toward resilience. Stigma and Health, 1(s), 60-74. Retrieved from https://psycnet.apa.org/journals/sah/1/S/60/

Ekama, S.O., Herbertson, E.C., Addeh, E.J., Gab-Okafor, C.V., Onwujekwe, D.I., Tayo, F., Ezechi, O. C. (2012). Pattern and determinants of antiretroviral drug adherence among Nigerian pregnant women. Journal of Pregnancy, 2012, 1-6. https://doi. org/10.1155/2012/851810

Fife, B. L., \& Wright, E. R. (2000). The dimensionality of stigma: A comparison of its impact on the self of persons with HIV/AIDS and cancer. Journal of Health and Social Behavior, 41(1), 50. https://doi.org/10.2307/2676360

French, G. L. (2005). Clinical impact and relevance of antibiotic resistance. Advanced Drug Delivery Reviews, 57(10), 1514-1527. https://doi.org/10.1016/J.ADDR.2005.04.005

French, H., Greeff, M., \& Watson, M. J. (2015). Experiences of people living with HIV and people living close to them of a comprehensive HIV stigma reduction community intervention in an urban and a rural setting. Sahara J, 11(1), 105-115. https://doi.org/ 
10.1080/17290376.2014.938104

Futterman, D., Shea, J., Besser, M., Stafford, S., Desmond, K., Comulada, W.S., Greco, E. (2010). Mamekhaya: a pilot study combining a cognitive-behavioral intervention and mentor mothers with PMTCT services in South Africa. AIDS Care, 22(9), 1093-1100. https://doi.org/10.1080/09540121003600352

Gaillard, P., Melis, R., Mwanyumba, F., Claeys, P., Muigai, E., Mandaliya, K., ... Temmerman, M. (2002). Vulnerability of women in an African setting: lessons for mother-tochild HIV transmission prevention programmes. AIDS, 16(6), 937-939. https://doi. org/10.1097/00002030-200204120-00019

Geldsetzer, P., Yapa, H. M. N., Vaikath, M., Ogbuoji, O., Fox, M. P., Essajee, S. M., ... Bärnighausen, T. (2016). A systematic review of interventions to improve postpartum retention of women in PMTCT and ART care. Journal of the International AIDS Society, 19(1). https://doi.org/10.7448/IAS.19.1.20679

Gelles, R. J., \& Straus, M. A. (1979). Violence in the American Family. Journal of Social Issues, 35(2), 15-39. https://doi.org/10.1111/j.1540-4560.1979.tb00799.x

Gertsch, A., Michel, O., Locatelli, I., Bugnon, O., Rickenbach, M., Cavassini, M., \& Schneider, M.-P. (2013). Adherence to antiretroviral treatment decreases during postpartum compared to pregnancy: A longitudinal electronic monitoring study. AIDS Patient Care and STDs, 27(4), 208-210. https://doi.org/10.1089/apc.2013.0005

Giordano, T.P., Guzman, D., Clark, R., Charlebois, E.D., Bangsberg, D. R. (2004). Measuring adherence to antiretroviral therapy in a diverse population using a visual analogue scale. HIV Clinical Trials, 5(2), 74-79. https://doi.org/10.1310/JFXH-G3X2-EYM6-D6UG

Hardon, A., Vernooij, E., Bongololo-Mbera, G., Cherutich, P., Desclaux, A., Kyaddondo, D., Ky-Zerbo, O., Neuman, M., Wanyenze, R., Obermeyer, C. (2012). Women's views on consent, counseling and confidentiality in PMTCT: a mixed-methods study in four African countries. BMC Public Health, 12(1), 26. https://doi.org/10.1186/1471-2458$12-26$

Hasan, T., Nath, S. R., Khan, N. S., Akram, O., Gomes, T. M., \& Rashid, S. F. (2012). Internalized HIV / AIDS-related stigma in a sample of hIV-positive people in bangladesh. Journal of Health, Population and Nutrition, 30(1), 22-30.

Hays, R. B., Turner, H., \& Coates, T. J. (1992). Social support, AIDS-related symptoms, and depression among gay men. Journal of Consulting and Clinical Psychology, 60(3), 463469. http://dx.doi.org/10.1037/0022-006X.60.3.463

Health Systems Trust. (2018). District Health Barometer 2016/2017. Retrieved from http:// www.hst.org.za/publications/Pages/District-Health-Barometer-201617.aspx

Hecht, F.M., Wang, L., Collier, A., Little, S., Markowitz, M., Margolick, J., Kilby, J.M., Daar, E., Conway, B., Holte, S. (2006). A multicenter observational study of the potential benefits of initiating combination antiretroviral therapy during acute HIV infection. The Journal of Infectious Diseases, 194(6), 725-733. https://doi.org/10.1086/506616

Hecht, F., Colfax, G., Swanson, M., \& Chesney, M. (1998). Adherence and effectiveness of protease inhibitors in clinical practice. Fifth Conference on Retroviruses and Opportunistic Infections. Chicago.

Heestermans, T., Browne, J. L., Aitken, S. C., Vervoort, S. C., \& Klipstein-Grobusch, K. (2016). Determinants of adherence to antiretroviral therapy among HIV-positive adults in 
sub-Saharan Africa: A systematic review. BMJ Global Health, 1(4), 1-13. https://doi. org/10.1136/bmjgh-2016-000125

Holzemer, W. L., Uys, L., Makoae, L., Stewart, A., Phetlhu, R., Dlamini, P. S., ... Naidoo, J. (2007). A conceptual model of HIV/AIDS stigma from five African countries. Journal of Advanced Nursing, 58(6), 541-551. https://doi.org/10.1111/j.1365-2648.2007.04244.x

Holzemer, W. L., \& Uys, L. R. (2004). Managing AIDS stigma. Journal of Social Aspects of HIV/ AIDS , 1(3), 165-174. Retrieved from https://www.ajol.info/index.php/saharaj/article/ viewFile/30082/22732

Horne, R., Buick, D., Fisher, M., Leake, H., Cooper, V., Weinman, J. (2004). Doubts about necessity and concerns about adverse effects: identifying the types of beliefs that are associated with non-adherence to HAART. International Journal of STD \& AIDS, 15(1), 38-44. https://doi.org/10.1258/095646204322637245

Horne, R., Chapman, S.C.E., Parham, R., Freemantle, N., Forbes, A., Cooper, V. (2013). Understanding patients' adherence-related beliefs about medicines prescribed for long-term conditions: A meta-analytic review of the necessity-concerns rramework. PLoS ONE, 8(12), e80633. https://doi.org/10.1371/journal.pone.0080633

Horne, R., Weinman, J., Hankins, M. (1999). The beliefs about medicines questionnaire: The development and evaluation of a new method for assessing the cognitive representation of medication. Psychology \& Health, 14(1), 1-24. https://doi. org/10.1080/08870449908407311

I.B.M Corp. (2016). IBM SPSS Statistics for Windows. Armonk: I.B.M Corp.

International HIV/AIDS Alliance. (2005). Antiretroviral (ARV) treatment fact sheet 02 ARV treatment. I SAFAIDS. Retrieved May 20, 2019, from Brighton, International HIV/ AIDS Alliance website: http://catalogue.safaids.net/publications/antiretroviral-arvtreatment-fact-sheet-02-arv-treatment

Jackson, A., Moyle, G., Watson, V., Tjia, J., Ammara, A., Back, D., Mohabeer, M., Gazzard, B., Boffito, M. (2013). Tenofovir, emtricitabine intracellular and plasma, and efavirenz plasma concentration decay following drug intake cessation. JAIDS Journal of Acquired Immune Deficiency Syndromes, 62(3), 275-281. https://doi.org/10.1097/ QAI.0b013e3182829bd0

Jalali, F.,Hasani, A., Hashemi, S.F., Kimiaei, S.A., \& Babaei, A. (2019). Cognitive group therapy based on schema-focused approach for reducing depression in prisoners living with HIV. International Journal of Offender Therapy and Comparative Criminology, 63(2), 276-288. https://doi.org/10.1177/0306624X18784185

Johnson, L. F. (2012). Access to antiretroviral treatment in South Africa , 2004 - 2011. The Southern African Journal of HIV Medicine, 13(1), 2004-2011.

Jones, D.L., Peltzer, K., Weiss, S.M., Sifunda, S., Dwane, N., Ramlagan, S., Cook, R., Matseke, G., Maduna, V., Spence, A. (2014). Implementing comprehensive prevention of motherto-child transmission and HIV prevention for South African couples: Study protocol for a randomized controlled trial. Trials, 15(1), 1-9. https://doi.org/10.1186/1745-6215$15-417$

Kadowa, I., \& Nuwaha, F. (2009). Factors influencing disclosure of HIV positive status in Mityana district of Uganda. African Health Sciences, 9(1), 26-33. Retrieved from https://www.ajol.info/index.php/ahs/article/view/7099 
Kako, P. M., \& Dubrosky, R. (2013). "You Comfort Yourself and Believe in Yourself": Exploring lived experiences of stigma in HIV-positive Kenyan women. Issues in Mental Health Nursing, 34(3), 150-157. https://doi.org/10.3109/01612840.2012.740765

Kalichman, S.C., Simbayi, L.C., Jooste, S., Toefy, Y., Cain, D., Cherry, C., Kagee, A. (2005). Development of a brief scale to measure AIDS-related stigma in South Africa. AIDS and Behavior, 9(2), 135-143. https://doi.org/10.1007/s10461-005-3895-x

Kalichman, S. C., Amaral, C. M., Swetzes, C., Jones, M., Macy, R., Kalichman, M. O., \& Cherry, C. (2009). A simple single-item rating scale to measure medication adherence: further evidence for convergent validity. Journal of the International Association of Physicians in AIDS Care (Chicago, III. : 2002), 8(6), 367-374. https://doi. org/10.1177/1545109709352884

Kastrissios, H., Suárez, J.R., Katzenstein, D., Girard, P., Sheiner, L.B., Blaschke, T. F. (1998). Characterizing patterns of drug-taking behavior with a multiple drug regimen in an AIDS clinical trial. AIDS (London, England), 12(17), 2295-2303. Retrieved from http:// www.ncbi.nlm.nih.gov/pubmed/9863872

Kilewo, C., Massawe, A., Lyamuya, E., Semali, I., Kalokola, F., Urassa, E., ... Biberfeld, G. (2001). HIV counseling and testing of pregnant women in Sub-Saharan Africa. JAIDS Journal of Acquired Immune Deficiency Syndromes, 28(5), 458-462. https://doi. org/10.1097/00042560-200112150-00009

Kingori, C., Reece, M., Obeng, S., Murray, M., Shacham, E., Dodge, B., ... Ojakaa, D. (2012). Impact of internalized stigma on HIV prevention behaviors among HIV-infected individuals seeking HIV care in Kenya. AIDS Patient Care and STDs, 26(12), 761-768. https://doi.org/10.1089/apc.2012.0258

Kirsten, I., Sewangi, J., Kunz, A., Dugange, F., Ziske, J., Jordan-Harder, B., ... Theuring, S. (2011). Adherence to combination prophylaxis for prevention of mother-to-childtransmission of HIV in Tanzania. PloS One, 6(6), e21020. https://doi.org/10.1371/ journal.pone.0021020

Kiula, E. S., Damian, D. J., \& Msuya, S. E. (2013). Predictors of HIV serostatus disclosure to partners among HIV-positive pregnant women in Morogoro, Tanzania. BMC Public Health, 13(1), 433. https://doi.org/10.1186/1471-2458-13-433

Kreitchmann, R., Harris, D. R., Kakehasi, F., Haberer, J. E., Cahn, P., Losso, M., ... Read, J. S. (2012). Antiretroviral adherence during pregnancy and postpartum in Latin America. AIDS Patient Care and STDs, 26(8), 486-495. https://doi.org/10.1089/apc.2012.0013

Kuhn, L., Semrau, K., Ramachandran, S., Sinkala, M., Scott, N., Kasonde, P., Mwiya, M., Kankasa, C., Decker, D., Thea, D.M., Aldrovandi, G. M. (2009). Mortality and virologic outcomes after access to antiretroviral therapy among a cohort of HIVinfected women who received single-dose nevirapine in Lusaka, Zambia. Journal of Acquired Immune Deficiency Syndromes, 52(1), 132-136. https://doi.org/10.1097/ QAI.0b013e3181ab6d5e

Kuonza, L., Tint, K. S., Harris, B., \& Nabukenya, I. (2011). Public health systems strengthening in Africa: the role of South Africa Field Epidemiology and Laboratory Training Programme. The Pan African Medical Journal, 10 Supp 1, 8. Retrieved from http:// www.ncbi.nlm.nih.gov/pubmed/22359696

Lawrie, T. A., Hofmeyr, G. J., de Jager, M., \& Berk, M. (1998). Validation of the Edinburgh Postnatal Depression Scale on a cohort of South African women. South African 
Medical Journal, 88(10), 1340-1344. Retrieved from http://www.ncbi.nlm.nih.gov/ pubmed/9807193

Lee, R. S., Kochman, A., \& Sikkema, K. J. (2002). Internalized Stigma Among People Living with HIV-AIDS.AIDSandBehavior,6(4),309-319.https://doi.org/10.1023/A:1021144511957

Lekas, H.-M., Siegel, K., \& Schrimshaw, E. W. (2006). Continuities and discontinuities in the experiences of felt and enacted stigma among women with HIV/AIDS. Qualitative Health Research, 16(9), 1165-1190. https://doi.org/10.1177/1049732306292284

Li, Z., \& Sheng, Y. (2014). Investigation of perceived stigma among people living with human immunodeficiency virus / acquired immune deficiency syndrome in Henan. International Journal of Nursing Sciences, 1(4), 385-388. https://doi.org/10.1016/j. ijnss.2014.10.019

Lindberg, M. H., Wettergren, L., \& Wiklander, M. (2014). Psychometric evaluation of the HIV stigma scale in a Swedish context. PLOS ONE, 9(12), 1-16. https://doi.org/10.1371/ journal.pone.0114867

Lohse, N., Hansen, A. B. E., Gerstoft, J., \& Obel, N. (2007). Improved survival in HIV-infected persons: Consequences and perspectives. Journal of Antimicrobial Chemotherapy, 60(3), 461-463. https://doi.org/10.1093/jac/dkm241

Loutfy, M. R., Logie, C. H., Zhang, Y., Blitz, S. L., Margolese, S. L., Tharao, W. E., ... Raboud, J. M. (2012). Gender and ethnicity differences in HIV-related stigma experienced by people living with HIV in Ontario, Canada. PLoS ONE, 7(12), e48168. https://doi.org/10.1371/ journal.pone.0048168

Magadi, M. A. (2011). Understanding the gender disparity in HIV infection across countries in sub-Saharan Africa: evidence from the Demographic and Health Surveys. Sociology of Health \& Illness, 33(4), 522-539. https://doi.org/10.1111/j.1467-9566.2010.01304.x

Makin, J. D., Forsyth, B. W. C., Visser, M. J., Sikkema, K. J., Ph, D., Neufeld, S., ... Mmed, O. (2008). Factors affecting disclosure in South African HIV-positive pregnant women. AIDS Patient Care and STDs, 22(11). https://doi.org/10.1089/apc.2007.0194

Mao, Y., Qiao, S., Li, X., Zhao, Q., Zhou, Y., \& Shen, Z. (2019). Depression, social support, and adherence to antiretroviral therapy among people living with HIV in Guangxi, China: A Longitudinal Study. AIDS Education and Prevention, 31(1), pp. 38-50. https://doi. org/10.1521/aeap.2019.31.1.38

Maru, S., Datong, P., Selleng, D., Mang, E., Inyang, B., Ajene, A., ... Abimiku, A. (2009). Social determinants of mixed feeding behavior among HIV-infected mothers in Jos, Nigeria. AIDS Care, 21(9), 1114-1123. https://doi.org/10.1080/09540120802705842

Matseke, G., Rodriguez, V. J., Peltzer, K., \& Jones, D. (2016). Intimate partner violence among HIV positive pregnant women in South Africa. Journal of Psychology in Africa, 26(3), 259-266. https://doi.org/10.1080/14330237.2016.1185912

Matseke, M. G., Ruiter, R.A.C., Rodriguez, V. J., Peltzer, K., Setswe, G., \& Sifunda, S. (2017). Factors Associated with Male Partner Involvement in Programs for the Prevention of Mother-to-Child Transmission of HIV in Rural South Africa. International journal of environmental research and public health, 14(11), 1333. doi:10.3390/ijerph14111333

Max, B., \& Sherer, R. (2000). Management of the adverse effects of antiretroviral therapy and medication adherence. Clinical Infectious Diseases, 30(Supplement 2), S96-S116. https://doi.org/10.1086/313859 
Mbonye, M., Nakamanya, S., Birungi, J., King, R., Seeley, J., \& Jaffar, S. (2013). Stigma trajectories among people living with HIV (PLHIV) embarking on a life time journey with antiretroviral drugs in Jinja, Uganda. BMC Public Health, 13(1), 804. https://doi. org/10.1186/1471-2458-13-804

Medley, A., Garcia-moreno, C., Mcgill, S., \& Maman, S. (2004). Rates, barriers and outcomes of HIV serostatus disclosure among women in developing countries: Implications for prevention of mother-to-child transmission programmes. Bulletin of the World Health Organization, 82(03), 299-307. Retrieved from https://www.scielosp.org/scielo. php?pid=S0042-96862004000400013\&script=sci_arttext\&tlng=en

Mehta, S., Moore, R.D., Graham, N. (1997). Potential factors affecting adherence with HIV therapy. AIDS, 11(14), 1665-1670. Retrieved from http://www.ncbi.nlm.nih.gov/ pubmed/9386800

Mellins, C.A., Chu, C., Malee, K., Allison, S., Smith, R., Harris, L., Higgins, A., Zorrilla, C., Landesman, S., Serchuck, L., Larussa, P. (2008). Adherence to antiretroviral treatment among pregnant and postpartum HIV-infected women. AIDS Care, 20(8), 958-968. https://doi.org/10.1080/09540120701767208

Mellins, C. A., Chu, C., Malee, K., Allison, S., Smith, R., Harris, L., ... Larussa, P. (2008). Adherence to antiretroviral treatment among pregnant and postpartum HIV-infected women. AIDS Care, 20(8), 958-968. https://doi.org/10.1080/09540120701767208

Mepham, S., Zondi, Z., Mbuyazi, A., Mkhwanazi, N., \& Newell, M. L. (2011). Challenges in PMTCT antiretroviral adherence in northern KwaZulu-Natal, South Africa. AIDS Care, 23(6), 741-747. https://doi.org/10.1080/09540121.2010.516341

MPAC. (2017). Mpumalanga Provincial Implementation Plan for HIV, TB and STIs 2017 2022. Nelspruit: MPAC.

Mpondo, F. (2018). The development of a culturally tailored and contextually sensitive behavior change intervention for heterosexual Xhosa-speaking women in the Eastern Cape province, South Africa. Maastricht University.

Mugoya, G. C. T., \& Ernst, K. (2014). Gender differences in HIV-related stigma in Kenya. AIDS Care, 26(2), 206-213. https://doi.org/10.1080/09540121.2013.808733

Muthén, L., \& Muthén, B. (2014). Mplus. Los Angeles: MPlus.

Mwaura, P. N. (2008). Stigmatization and Discrimination of HIV/AIDS Women in Kenya: A Violation of Human Rights and its Theological Implications. Exchange, 37(1), 35-51. https://doi.org/10.1163/157254308X251322

Nachega, J.B., Marconi, V.C., van Zyl, G.U., Gardner, E.M., Preiser, W., Hong, S.Y., Mills, E.J., Gross, R. (2011). HIV treatment adherence, drug resistance, virologic failure: evolving concepts. Infectious Disorders Drug Targets, 11(2), 167-174. Retrieved from http:// www.ncbi.nlm.nih.gov/pubmed/21406048

Nachega, J.B., Uthman, O.A., Anderson, J., Peltzer, K., Wampold, S., Cotton, M.F., Mills, E.J., Ho, Y.S., Stringer, J.S.A., McIntyre, J.A., Mofenson, L. M. (2012). Adherence to antiretroviral therapy during and after pregnancy in low-income, middle-income, and high-income countries: A systematic review and meta-analysis. Aids, 26(16), 20392052. https://doi.org/10.1097/QAD.0b013e328359590f

Nebié, Y., Meda, N., Leroy, V., Mandelbrot, L., Yaro, S., Sombié, I., ... Dabis, F. (2001). Sexual and reproductive life of women informed of their HIV seropositivity: A prospective 
cohort study in Burkina Faso. JAIDS Journal of Acquired Immune Deficiency Syndromes, 28(4), 367-372. https://doi.org/10.1097/00126334-200112010-00010

Ngidi, W. H., Naidoo, J. R., Ncama, B. P., Luvuno, Z. P. B., \& Mashamba-Thompson, T. P. (2017). Mapping evidence of interventions and strategies to bridge the gap in the implementation of the prevention of mother-to-child transmission of HIV programme policy in sub-Saharan countries: A scoping review. African Journal of Primary Health Care and Family Medicine, 9(1). https://doi.org/10.4102/phcfm.v9i1.1368

NIMH Multisite HIV/STD Prevention Trial for African American Couples Group. (2008). Designing an audio computer-assisted self-interview system in a multisite trial: A brief report. Journal of Acquired Immune Deficiency Syndromes, 49(1), S52-S58. https:// doi.org/10.1016/j.freeradbiomed.2008.10.025.The

Novick, A. (1997). Stigma and AIDS: Three layers of damage. Journal of the Gay and Lesbian Medical Association, 1(1), 53-60. https://doi.org/10.1023/B:JOLA.0000007011.17392. $\mathrm{fb}$

Olley, B. O., Zeier, M. D., Seedat, S., \& Stein, D. J. (2005). Post-traumatic stress disorder among recently diagnosed patients with HIV/AIDS in South Africa. AIDS Care, 17(5), 550-557. https://doi.org/10.1080/09540120412331319741

Onyebuchi-iwudibia, O., \& Brown, A. (2014). HIV and depression in Eastern Nigeria : The role of HIV-related stigma. AIDS Care, 26(5), 653-657. https://doi.org/10.1080/09540121 .2013.844761

Patel, R., Ratner, J., Gore-Felton, C., Kadzirange, G., Woelk, G., Katzenstein, D. (2012). HIV disclosure patterns, predictors, and psychosocial correlates among HIV positive women in Zimbabwe. AIDS Care, 24(3), 358-368. https://doi.org/10.1080/09540121 .2011 .608786

Paterson, D. L., Swindells, S., Mohr, J., Brester, M., Vergis, E. N., Squier C., ... Hudson, B. (2000). Adherence to protease inhibitor therapy and outcomes in patients with $\{$ HIV infection. Annals of Internal Medicine, 133(1), 21-30. https://doi.org/10.7326/00034819-133-1-200007040-00004

Peltzer, K., Abbamonte, J. M., Mandell, L. N., Rodriguez, V. J., Lee, T. K., Weiss, S. M., \& Jones, D. L. (2019). The effect of male involvement and a prevention of mother-tochild transmission (PMTCT) intervention on depressive symptoms in perinatal HIVinfected rural South African women. Archives of Women's Mental Health. https://doi. org/10.1007/s00737-019-00955-7

Peltzer, K., Babayigit, S., Rodriguez, V. J., Jean, J., Sifunda, S., \& Jones, D. L. (2018). Effect of a multicomponent behavioural PMTCT cluster randomised controlled trial on HIV stigma reduction among perinatal HIV positive women in Mpumalanga province, South Africa. SAHARA J : Journal of Social Aspects of HIV/AIDS Research Alliance, 15(1), 80-88. https://doi.org/10.1080/17290376.2018.1510787

Peltzer, K., \& Mlambo, G. (2013). HIV disclosure among HIV positive new mothers in South Africa. Journal of Psychology in Africa, 23(2), 327-333. https://doi.org/10.1080/1433 0237.2013.10820631

Peltzer, K., Sikwane, E., \& Majaja, M. (2011). Factors associated with short-course antiretroviral prophylaxis (dual therapy) adherence for PMTCT in Nkangala district, South Africa. Acta Paediatrica, 100(9), 1253-1257. https://doi.org/10.1111/j.16512227.2011.02253.x 
Peltzer, K., Weiss, S. M., Soni, M., Lee, T. K., Rodriguez, V. J., Cook, R., ... Jones, D. L. (2017). A cluster randomized controlled trial of lay health worker support for prevention of mother to child transmission of HIV (PMTCT) in South Africa. AIDS Research and Therapy, 14(1), 1-12. https://doi.org/10.1186/s12981-017-0187-2

Phillips, L. A., Diefenbach, M. A., Kronish, I. M., Negron, R. M., \& Horowitz, C. R. (2014). The necessity-concerns framework: a multidimensional theory benefits from multidimensional analysis. Annals of Behavioral Medicine, 48(1), 7-16. https://doi. org/10.1007/s12160-013-9579-2

Pinkerton, S. D., \& Galletly, C. L. (2007). Reducing HIV Transmission Risk by Increasing Serostatus Disclosure: A Mathematical Modeling Analysis. AIDS and Behavior, 11(5), 698-705. https://doi.org/10.1007/s10461-006-9187-2

Rahangdale, L., Banandur, P., Sreenivas, A., Turan, J. M., Washington, R., \& Cohen, C. R. (2010). Stigma as experienced by women accessing prevention of parent-to-child transmission of HIV services in Karnataka, India. AIDS Care, 22(7), 836-842. https:// doi.org/10.1080/09540120903499212

Ramirez-Ferrero, E., \& Lusti-Narasimhan, M. (2012). The role of men as partners and fathers in the prevention of mother-to-child transmission of HIV and in the promotion of sexual and reproductive health. Reproductive Health Matters, 20(sup39), 103-109. https://doi.org/10.1016/S0968-8080(12)39642-0

Ramlagan, S., Peltzer, K., Ruiter, R. A. C., Barylski, N. A., Weiss, S. M., \& Sifunda, S. (2018). Prevalence and factors associated with fixed-dose combination antiretroviral drugs adherence among HIV-positive pregnant women on option b treatment in Mpumalanga province, South Africa. International Journal of Environmental Research and Public Health, 15(1), 1-12. https://doi.org/10.3390/ijerph15010161

Rankin, W. W., Brennan, S., Schell, E., Laviwa, J., \& Rankin, S. H. (2005). The stigma of being HIV-positive in Africa. PLoS Medicine, 2(8), e247. https://doi.org/10.1371/journal. pmed.0020247

Rao, D., Desmond, M., Andrasik, M., Rasberry, T., Lambert, N., Cohn, S. E., \& Simoni, J. (2012). Feasibility, Acceptability, and Preliminary Efficacy of the Unity Workshop: An Internalized Stigma Reduction Intervention for African American Women Living with HIV. AIDS Patient Care and STDs, 26(10), 614-620. https://doi.org/10.1089/ apc.2012.0106

Reda, A. A., \& Biadgilign, S. (2012). Determinants of adherence to antiretroviral therapy among HIV-infected patients in Africa. AIDS Research and Treatment, 2012, 1-8. https://doi.org/10.1155/2012/574656

Remien, R. H. (1998). Adhering to HIV combination therapy: The role of the pharmacist. Pharmacy Times, May, 28-37. Retrieved from https://scholar.google.com/ scholar_lookup?title=Adhering to HIV combination therapy\%3A The role of the pharmacist\&author=R. H.. Remien\&journal=Pharmacy Times\&volume=1998\&issue= May\&pages=28-37\&publication_year=1998

Robbins, R. N., Spector, A. Y., Mellins, C. A., \& Remien, R. H. (2014). Optimizing ART adherence: Update for HIV treatment and prevention. Current HIV/AIDS Reports, Vol. 11, pp. 423-433. https://doi.org/10.1007/s11904-014-0229-5

Roberts, K.J., \& Mann, T. (2000). Barriers to antiretroviral medication adherence in HIV-infected women. AIDS Care, 12(4), 377-386. https://doi.org/10.1080/09540120050123774 
Rollins, N.C., Coovadia, H.M., Bland, R.M., Coutsoudis, A., Bennish, M.L., Patel, D., \& Newell, M.-L. (2007). Pregnancy outcomes in HIV-infected and uninfected women in rural and urban South Africa. Journal of Acquired Immune Deficiency Syndromes, 44(3), 321-328. Retrieved from http://ovidsp.ovid.com/ovidweb.cgi?T=JS\&PAGE= reference $\& D=e m e d 11 \& N E W S=N \& A N=46328350$

Sagay, A., Musa, J., Ekwempu, C., \& Imade, G. (2006). Partner Disclosure of HIV Status Among HIV Positve Mothers in Northern Nigeria. African Journal of Medicine and Medical Sciences, 35(119-23). Retrieved from http://irepos.unijos.edu.ng/jspui/ handle/123456789/849

SANAC. (2016). LET OUR ACTIONS COUNT: Reflections on NSP 2012-2016 and Moving forward to NSP 2017-2022. Retrieved from http://nsp.sanac.org.za/wp-content/ uploads/2017/01/Final-NSP-Document.pdf

Sandelowski, M., Lambe, C., \& Barroso, J. (2004). Stigma in HIV-Positive Women. Journal of Nursing Scholarship, 36(2), 122-128. https://doi.org/10.1111/j.15475069.2004.04024.x

Sangaramoorthy, T., Jamison, A., \& Dyer, T. (2017). Intersectional stigma among midlife and older Black women living with HIV. Culture, Health \& Sexuality, 19(12), 1329-1343. https://doi.org/10.1080/13691058.2017.1312530

Schouten, E. J., Jahn, A., Chimbwandira, F., Harries, A. D., \& Damme, W. Van. (2013). Is Option $\mathrm{B}+$ the best choice? The Lancet, 381(9874), 1272-1273. https://doi.org/10.1016/ S0140-6736(13)60833-8

Sekoni, A. O., Obidike, O. R., \& Balogun, M. R. (2012). Stigma, medication adherence and coping mechanism among people living with HIV attending General Hospital, Lagos Island, Nigeria. African Journal of Primary Health Care \& Family Medicine, 4(1), 10. https://doi.org/10.4102/phcfm.v4i1.417

Serovich J. M. (2001). A test of two HIV disclosure theories. AIDS education and prevention: official publication of the International Society for AIDS Education, 13(4), 355-364.

Shamu, S., Zarowsky, C., Shefer, T., Temmerman, M., \& Abrahams, N. (2014). Intimate partner violence after disclosure of HIV test results among pregnant women in Harare , Zimbabwe. PLoS ONE, 9(10). https://doi.org/10.1371/journal.pone.0109447

Shisana, O., Rehle, T., Simbay, L.C., Zuma, K., Jooste, S., Zungu, N., Labadarios, D. Onoya, D. (2014). South African National HIV Prevalence, Incidence and Behaviour Survey, 2012. HSRC Press, 194.

Shiyoleni, M. (2013). Non-disclosure of HIV serostatus to partners among HIV positive pregnant women enrolled on the prevention of mother to child transmission (PMTCT) program at Katutura antenatal clinic, Windhoek, Namimbia (Stellenbosch University). Retrieved from http://scholar.sun.ac.za/handle/10019.1/79858

Sifunda, S., Peltzer, K., Rodriguez, V. J., Mandell, L. N., Lee, T. K., Ramlagan, S., Alcaide, M.L., \& Jones, D. L. (2019). Impact of male partner involvement on mother-tochild transmission of HIV and HIV-free survival among HIV-exposed infants in rural South Africa: Results from a two phase randomised controlled trial. PloS one, 14(6), e0217467. doi:10.1371/journal.pone.0217467

Simbayi, L.C., Zuma, K., Zungu, N., Moyo, .S, Marinda, E., Jooste, S., Mabaso, M., Ramlagan, S., North, A., van Zyl, J., Mohlabane, N., Dietrich, C., Naidoo, I., A. S., \& Team. (2019). 
South African National HIV Prevalance, Incidence, Behaviour and Communication survey, 2017. Cape Town: HSRC Press.

Simbayi, L. C., Kalichman, S., Strebel, A., Cloete, A., Henda, N., \& Mqeketo, A. (2007). Internalized stigma, discrimination, and depression among men and women living with HIV/AIDS in Cape Town, South Africa. Social Science and Medicine, 64(9), 18231831. https://doi.org/10.1016/j.socscimed.2007.01.006

Simoni, J. M., Pantalone, D. W., Plummer, M. D., \& Huang, B. (2007). A randomized controlled trial of a peer support intervention targeting antiretroviral medication adherence and depressive symptomatology in HIV-positive men and women. Health Psychology, 26(4), 488-495. http://dx.doi.org/10.1037/0278-6133.26.4.488

Skinner, D., \& Mfecane, S. (2004). Stigma, discrimination and the implications for people living with HIV/AIDS in South Africa. SAHARA-J: Journal of Social Aspects of HIV/AIDS, 1(3), 157-164. https://doi.org/10.1080/17290376.2004.9724838

Ssali, S. N., Atuyambe, L., Tumwine, C., Segujja, E., Nekesa, N., Nannungi, A., ... Wagner, G. (2010). Reasons for disclosure of HIV status by people living with HIV/AIDS and in HIV care in Uganda: An exploratory study. AIDS Patient Care and STDs, 24(10), 675-681. https://doi.org/10.1089/apc.2010.0062

StatsSA. (2001). Data Census 2001: Investigation into appropriate definitions of urban and rural areas for South Africa: Discussion document. In StatsSA (Vol. 1). Retrieved from http://www.statssa.gov.za/census/census_2001/urban_rural/urbanrural.pdf

StatsSA. (2018). Mid-year population estimates, 2018. Pretoria: STATSSA.

Straus, M. A. (1979). Measuring Intrafamily Conflict and Violence: The Conflict Tactics (CT) Scales. Journal of Marriage and the Family, 41(1), 75. https://doi.org/10.2307/351733

Tabana, H., Doherty, T., Swanevelder, S., Lombard, C., Jackson, D., Zembe, W., Naik, R. (2012). Knowledge of HIV status prior to a community HIV counseling and testing intervention in a rural district of south Africa: results of a community based survey. BMC Infectious Diseases, 12(1), 73. https://doi.org/10.1186/1471-2334-12-73

Tam, M., Amzel, A., \& Phelps, B. R. (2015). Disclosure of HIV serostatus among pregnant and postpartum women in sub-Saharan Africa: A systematic review. AIDS Care, 27(4), 436-450. https://doi.org/10.1080/09540121.2014.997662

Tenthani, L., Haas, A.D., Tweya, H., Jahn, A., Van Oosterhout, J.J., Chimbwandira, F., Chirwa, Z., Ng'Ambi, W., Bakali, A., Phiri, S., Myer, L., Valeri, F., Zwahlen, M., Wandeler, G., Keiser, O. (2014). Retention in care under universal antiretroviral therapy for HIVinfected pregnant and breastfeeding women ('Option B+') in Malawi. Aids, 28(4), 589598. https://doi.org/10.1097/QAD.0000000000000143

Tsai, A. C. (2015). Socioeconomic gradients in internalized stigma among 4,314 persons with HIV in sub-Saharan Africa. AIDS and Behavior, 19(2), 270-282. https://doi. org/10.1007/s10461-014-0993-7

Tsai, A. C., Bangsberg, D. R., \& Weiser, S. D. (2013). Harnessing poverty alleviation to reduce the stigma of HIV in Sub-Saharan Africa. PLoS Medicine, 10(11), e1001557. https://doi. org/10.1371/journal.pmed.1001557

Turan, J.M., Bukusi, E.A., Onono, M., Holzemer, W.L., Miller, S., Cohen, C. R. (2011). HIV/AIDS stigma and refusal of HIV testing among pregnant women in rural Kenya: Results from the MAMAS study. AIDS and Behavior, 15(6), 1111-1120. https://doi.org/10.1007/ 
s10461-010-9798-5

Turan, J.M., Hatcher, A.H., Medema-Wijnveen, J., Onono, M., Miller, S., Bukusi, E.A., Turan, B. (2012). The role of HIV-related stigma in utilization of skilled childbirth services in rural Kenya: A prospective mixed-methods study. PLoS Medicine, 9(8), e1001295. https://doi.org/10.1371/journal.pmed.1001295

UNAIDS. (n.d.). Gender \& AIDS fact sheets : Rural HIV/AIDS. Retrieved from http://data. unaids.org/topics/gender/ruralhivaids_en.pdf

UNAIDS. (2011). Countdown to zero: global plan towards the elimination of new HIV infections among children by 2015 and keeping their mothers alive, 2011-2015. Geneva: UNAIDS.

UNAIDS. (2014). The GAP report. Retrieved from http://www.refworld.org/docid/53f1e1604. html

UNAIDS. (2015). UNAIDS Terminology Guidelines. Geneva.

UNAIDS. (2017a). Ending AIDS: Progress towards 90-90-90 targets. (UNAIDS, Ed.). Geneva: UNAIDS.

UNAIDS. (2017b). Fact Sheet-World AIDS Day 2017. Retrieved November 30, 2017, from http://www.unaids.org/en/resources/fact-sheet

UNAIDS. (2018). UNAIDS Data 2018. In Programme on HIV/AIDS. Retrieved from http://www. unaids.org/sites/default/files/media_asset/unaids-data-2018_en.pdf\%0Ahttp:// www.unaids.org/sites/default/files/media_asset/20170720_Data_book_2017_ en.pdf

UNICEF. (2012). Options B and B+: Key considerations for countries to implement an equityfocused approach. Eliminating New HIV Infections among Children and Keeping Mothers Living with HIV Alive andWell. In UNICEF (Vol. 6). Retrieved from https:// www.unicef.org/aids/files/hiv_\%0AKey_considerations_options_B.pdf

Van Damme, L., Corneli, A., Ahmed, K., Agot, K., Lombaard, J., Kapiga, S., ... FEM-PrEP Study Group. (2012). Preexposure prophylaxis for HIV infection among African Women. New England Journal of Medicine, 367(5), 411-422. https://doi.org/10.1056/ NEJMoa1202614

van Servellen, G., Johiro, A. K., \& Tichacek, M. J. (2002). Detection and documentation of actual and potential medication adherence problems in patients receiving combination therapies. Journal of the Association of Nurses in AIDS Care, 13(4), 64-77. https://doi. org/10.1016/S1055-3290(06)60372-5

Volk, J.E., Marcus, J.L., Phengrasamy, T., Blechinger, D., Nguyen, D.P., Follansbee, S., Hare, C. B. (2015). No new HIV infections with increasing use of HIV preexposure prophylaxis in a clinical practice setting. Clinical Infectious Diseases, 61(10), 1601-1603. https://doi. org/10.1093/cid/civ778

Wagenaar, B. H., Sullivan, P. S., \& Stephenson, R. (2012). HIV knowledge and associated factors among internet-using men who have sex with men (MSM) in south africa and the united states. PLOS ONE, 7(3), 1-8. https://doi.org/10.1371/journal.pone.0032915

Wahl, L. M., \& Nowak, M. A. (2000). Adherence and drug resistance: Predictions for therapy outcome. Proceedings of the Royal Society B: Biological Sciences, 267(1445), 835-843. https://doi.org/10.1098/rspb.2000.1079

Watson, S. (2019). Antiretroviral HIV drugs: Side effects and adherence. Retrieved May 
21, 2019, from Healthline website: https://www.healthline.com/health/hiv-aids/ antiretroviral-drugs-side-effects-adherence

Weiser, S.D., Tuller, D.M., Frongillo, E.A., Senkungu, J., Mukiibi, N., Bangsberg, D. R. (2010). Food insecurity as a barrier to sustained antiretroviral therapy adherence in Uganda. PloS One, 5(4), e10340. https://doi.org/10.1371/journal.pone.0010340

Wettstein, C., Mugglin, C., Egger, M., Blaser, N., Salazar, L., Estill, J., Bender, N., Davies, M., Wandeler, G. (2012). Missed opportunities to prevention mother-to-child-transmission in sub-Saharan Africa : systematic review and meta-analysis. Aids, 26(18), 2361-2373. https://doi.org/10.1097/QAD.0b013e328359ab0c.Missed

WHO/UNAIDS/UNICEF. (2010). Towards universal access: Scaling up Priority HIV/AIDS Interventions in the Health Sector. In UNAIDS Annual Report. Retrieved from http:// www.unaids.org/en/KnowledgeCentre/Resources/Publications/default.asp

WHO. (2010). Programmatic Update: Use of Antiretroviral Drugs for Treating Pregnant Women and Preventing HIV INfection in Infants. In Geneva: WHO. https://doi.org/ WHO/HIV/2012.6

WHO. (2012a). Male involvement in the prevention of mother-to-child transmission of HIV. Retrieved from https://apps.who.int/iris/bitstream/ handle/10665/70917/9789241503679_eng.pdf

WHO. (2012b). Use of antiretroviral drugs for treating pregnant women and preventing HIV infection in infants. In WHO. Retrieved from World Health Organization website: https://www.who.int/hiv/pub/mtct/programmatic_update2012/en/

Williams, B., Wood, R., Dukay, V., Delva, W., Ginsburg, D., Hargrove, J., Stander, M., Sheneberger, R., Montaner, J., Welte, A. (2011). Treatment as prevention: preparing the way. Journal of the International AIDS Society, 14 Suppl 1(Suppl 1), S6. https://doi. org/10.1186/1758-2652-14-S1-S6

Yuh, J. N., Ellwanger, K., Potts, L., \& Ssenyonga, J. (2014). Stigma among HIV/AIDS Patients in Africa: A critical Review. Procedia - Social and Behavioral Sciences, 140, 581-585. https://doi.org/10.1016/J.SBSPRO.2014.04.474 
SUMMARY 


\section{SUMMARY}

South Africa has the highest HIV prevalence in the world, and although HIV is now regarded as a chronic condition, some HIV positive individuals still do not adhere to their antiretroviral therapy (ART). This thesis investigated the structural and psychosocial determinants of ART adherence among pregnant women in Mpumalanga province, South Africa. The five empirical studies that make up this thesis and the major recommendations are summarized below.

The first study of this thesis explored the prevalence of ART drugs adherence as well as the factors associated with ART drugs adherence among the study population. This study therefore sets the scene in understanding the study population group which consisted of 673 HIV-positive pregnant women, with a mean age of 28 years old. The study found high non-adherence to ART among both self-reported measures i.e $21 \%$ AACTG non-adherence and $31 \%$ VAS non-adherence. A number of covariates were found to contribute to nonadherence and include alcohol intake, the desire to avoid ART side effects, non-disclosure of ones HIV status, depression, and HIV knowledge. Older age was an important outcome for increased adherence to ART as the study found that respondents who were of an older age were more likely to be adherent than younger respondents in study sample.

The second study examined the pregnant woman's determinants of HIV-positive status disclosure and non-disclosure. ART adherence was used as a covariate to understand its association with disclosing ones HIV positive status to someone or to one's partner. High levels of overall non-disclosure of the respondents' HIV positive status was noted in this study as just over two fifths did not disclose their HIV positive status to their male partners and over a quarter did not disclose to someone. Non-disclosure of ones HIV positive status was strongly associated with non-adherence of ART. Multivariate analysis showed that both disclosure of ones HIV status to someone and to their male partners was significantly associated with increase in ART adherence, the known HIV positive status of their partner, and male involvement during pregnancy. Participants who were diagnosed HIV positive during this current pregnancy were less likely to disclose their HIV status to someone.

The third study explored the HIV positive pregnant women's socioeconomic, behavioral and HIV-related correlates of internalized stigma. In this study, ART adherence was used as a covariate to understand its association with each of the four internalized stigma factors: personalized stigma, concerns with public attitudes about people with HIV, disclosure concerns, and negative self-image. This study found that having no male partner involvement in the antenatal stage of the pregnancy was significantly associated with experiencing all four of the internalized stigma factors. IPV was found to be significantly associated with experiencing personalized stigma, concerns with public attitudes about people with HIV, and 
experiencing the stigma of negative self-image. Women whose household income was classified as low, were more likely to experience three of the four HIV-related stigma factors. Respondents that did not complete school, were also more likely to experience personalized stigma.

The fourth study compares the results from two self-reported adherence scales, namely the visual analog scale (VAS) (Giordano et al., 2004) and the Adult AIDS Clinical Trials Group (AACTG) (Chesney et al., 2000) scale, with the respondents dried blood spot (DBS) adherence measure. Comparing all three measures of adherence at 32 weeks pregnant, $86 \%$ of respondents' self-reported adherent on the VAS and $80 \%$ self-reported adherent on the AACTG, whereas $74 \%$ were found to be ART adherent according to their DBS result.

The final study examines the self-reported change in ART adherence over time and the reasons for missing ART. This study utilized the respondent's baseline (8-24 weeks pregnant) and 12 month post-partum data points to test an ART adherence intervention and understand ART adherence change over time. The intervention did not have the desired outcome on remaining adherent nor did it have an impact on becoming adherent over time, which was likely due to the significantly greater proportion of non-adherent women in the experimental condition than in the control condition at baseline (55 versus $46 \%$ ). An additional potential reason for intervention failure could stem from the study design of being a cluster-randomized trial. The study also find that there was a change to nonadherence over time and although a distressing intervention effect, the finding mimics reported longitudinal studies among HIV infected women from around the world. The necessity-concerns framework (Horne et al., 2004; Clifford et al., 2008) clarifies that the mother adhered to her ART regime as she deemed it necessary to protect the fetus from HIV and thus the concerns for the unborn baby outweighed the concerns about taking the medication. Once the child was born HIV-negative, the mother had no concern for the transfer of HIV from mother to child and therefore may not have been motivated to continue taking her ART, and post-partum ART adherence decreased. At this juncture, the concern about taking ART outweighed the necessity to take it. During the post-natal phase, high loss to follow-up was experienced. Both the high loss to follow up and limited ART adherence intervention during the post-natal phase of the study resulted in a lack of intervention for the majority of respondents. Reason given by the respondents in the enhanced intervention group for missing their ART include that they were away from their home when they needed to take their medication, having problems taking their medication at specified times and, having problems taking their medication due to the lack of food.

This thesis concludes with practical implications from its findings. These recommendations include: (1) the need for interventions to show the necessity and mitigate the concern of adhering to the ART regime post-partum, (2) better 
retention at clinic level by increasing clinic outreach, (3) creation of a nationally linked electronic patient file network where all patients can access health care in any public clinic/hospital in South Africa and have their health files readily available to them, (4) the need to encourage disclosure as a way to promote ART adherence, (5) increased psychosocial support for newly diagnosed, (6) encouraging male partner involvement during and after pregnancy, (7) support groups for both women and men need to be set up to mitigate IPV as well as to cope should it happen, (8) support groups for women to provide them with a safe space to talk through their issues and assist them with their depression, (9) ART adherence testing through DBS where needed, and (10) usage of the 40-item internalized stigma scale among other vulnerable populations in South Africa. 


\section{VALORISATION}




\section{VALORISATION}

The findings of this thesis will be explained in terms of its relevance at a societal level. In so doing, this section defines the group targeted by the practical implications of this thesis, the actual product and innovation that could be potentially gained from it, and the future planning and implementation that will be formulated.

South Africa has an estimated 8 million people living with HIV and has adopted a policy of providing anti-retroviral therapy (ART) medication to all those who are HIV positive no matter their CD4 or immune system count. This thesis sought to better understand adherence as well as non-adherence to ART among HIV positive pregnant women in Mpumalanga province, South Africa. In order to progress in the battle against the HIV epidemic, it is critical that people who are living with HIV/ AIDS (PLWHA) remain adherent to their ART regime which would lead to the virus becoming undetectable within them and thus they would become non-infectious. It this were to happen among pregnant women then it is very possible to have all babies born in South Africa to be born HIV negative and be sustained that way for the first year of their lives.

The thesis provides a number of practical implications aimed at different national and international role players that interact with pregnant PLWHA on a daily basis. Firstly, the South African Department of Health (DoH) would be instrumental in insuring a national linked computerized patient filing system is put in place so that patients and their health care providers can access their health records anywhere in South Africa and can thus provide the correct intervention as, when and where needed. In cases where needed, the DoH needs to ensure that ART adherence is also measured using dry blood spot (DBS). The thesis does highlight the expense of using DBS but states that it should be used only when needed to ensure adherence is maintained. The DoH can also ensure better patient retention by increasing its patient outreach program and also include pregnant and post-natal mothers into this outreach program.

The practical implications of this thesis are also aimed at national and international non-governmental organisations (NGOs) who would be instrumental at setting up and running the suggested psychosocial support groups. These should be done in conjunction with academics who would research best practice that is theory based and utilise intervention mapping to suggest intervention and then continually improve them for individual situations. Interventions for increasing ART adherence post-partum by potentially utilizing the necessity concerns framework also require further academic research. These sort of interventions either if government initiated or initiated by NGOs or academics require funding and thus international donors as well as the private sector need to be brought aboard. The findings of this thesis is therefore also aimed at them as a critical component. 
The five chapters that make up the empirical component of this thesis have already been published in international peer reviewed journals. Apart from these five articles, numerous other articles have been published by the research team and further articles are planned. The findings of the study will also be presented at international conferences as well as via electronic and paper based media briefs. As a large and important component of the recommendations involve the South African government, policy briefs, specifically aimed at the DoH, will be written to highlight the problems and recommendations. The study observations and findings have encouraged the research team to consider writing proposals and seek funding for increasing the uptake of PMTCT in South Africa, increasing the number of children on ART as well as to investigate the impact of ARVs on early childhood development. Concept notes are currently being explored. 



\section{ACKNOWLEDGEMENTS}

There have been many people who have been there for me in some capacity that enabled me to complete this PhD thesis. I would firstly like to thank Prof. Karl Peltzer who really took me under his wing and taught me about all aspects of research as well as analysis and has guided me, my career and my studies in so many ways that I will forever be grateful and will never forget. To Prof. Sibusiso Sifunda who had somehow got me into this $\mathrm{PhD}$ program by a simple corridor conversation and a cup of tea and then made this entire PhD process so easy while at the same time protecting me on the work front and always taking the time to listen no matter when even I went knocking - - all this afforded me the time and the security to complete my studies. To Prof. Rob Ruiter, my heartfelt gratitude goes to you for making this entire process so easy. Now a PhD is in no means easy, but as a supervisor, Rob, you were more than brilliant and I truly believe that you are the reason that I have this document. To my many, many friends and colleagues at the Human Sciences Research Council, thank you for being patient with me, for giving me time, for listening and most importantly, thank you for the gin.

To the most important people in my life, my family, words will never truly express how thankful I am. My cousin Narissa, who always pushed and encouraged me since childhood, thank you. To my mum, Shamin Ramlagan and my dad, Naleen Ramlagan, how does one begin to thank you guys ... I love you. To Tanya, my partner, the person who was there every step during university, the person who made me a dad, who supported my move into research, I thank you so very much and I love you. And to my greatest achievements, Inara and Niya, follow your hearts.

I really do hope that one day I will make you all proud by paying everything you have all given me, forward.

Thank you all, I final got there. 
CURRICULUM VITAE 


\section{CURRICULUM VITAE}

Shandir Ramlagan was born on June $23^{\text {rd }}, 1976$ in Durban, South Africa. He studied at the University of Natal where he completed his Bachelor of Social Science degree in 1997 and his Master of Development Studies in 2003. From 1999 to 2001, he worked at DRA Development, first as an intern and then as a junior researcher. In 2002, he joined the Human Sciences Research Council (HSRC) of South Africa as a junior research involved in the countries first national HIV prevalence and behavioural survey. He is currently a Senior Research Specialist in the Social Aspects of Public Health (SAPH) research unit of the HSRC. He has experience in social science research; both in the areas of qualitative and quantitative research design, research methodology, management of surveys as well as data analysis and report writing. His project experience includes working on all five of the South African National HIV Prevalence, Incidence, Behaviour and Communication surveys' in roles ranging from data collector to Project Director. He has worked on NIH R01 funded randomized controlled trials as well as on projects for organizations such as the W.K. Kellogg Foundation, Nelson Mandela Children's Fund, World Bank, Population Council, and various government departments. His publication record spans over 50 peer reviewed journal articles, project reports, co-authored book chapters, conceptual and epidemiological models. He is currently Managing Editor on the Journal of Social Aspects of HIV/AIDS Research Alliance (SAHARA-J). 

